TRANSACTIONS OF THE

AMERICAN MATHEMATICAL SOCIETY

Volume 353, Number 8 , Pages 3045-3095

S 0002-9947(01)02746-5

Article electronically published on January 18, 2001

\title{
COMPACTIFYING THE RELATIVE JACOBIAN OVER FAMILIES OF REDUCED CURVES
}

\author{
EDUARDO ESTEVES
}

\begin{abstract}
We construct natural relative compactifications for the relative Jacobian over a family $X / S$ of reduced curves. In contrast with all the available compactifications so far, ours admit a Poincaré sheaf after an étale base change. Our method consists of studying the étale sheaf $F$ of simple, torsion-free, rank1 sheaves on $X / S$, and showing that certain open subsheaves of $F$ have the completeness property. Strictly speaking, the functor $F$ is only representable by an algebraic space, but we show that $F$ is representable by a scheme after an étale base change. Finally, we use theta functions originating from vector bundles to compare our new compactifications with the available ones.
\end{abstract}

\section{INTRODUCTION}

0.1. History and goal. The problem of finding a natural relative compactification of the relative Jacobian over a family of curves has drawn a lot of attention since Igusa's pioneering work [17] in the fifties. After important work of Mayer, Mumford [22] and D'Souza [8], a very satisfactory solution has been found by Altman and Kleiman 3 in the case where the curves in the family are geometrically integral: their relative compactification is a fine moduli space, i.e. admits a universal, or Poincaré, sheaf after an étale base change. We refer to the introduction in [3] for more details.

Despite the progress for integral curves, the case of reducible curves (especially nodal ones) is important for applications, and had to be tackled. In the case of a single reduced (possibly reducible) nodal curve defined over an algebraically closed field, Oda and Seshadri 23 used Geometric Invariant Theory (GIT) to construct several compactifications of disjoint unions of copies of the Jacobian. Afterwards, Seshadri used GIT to deal with a general reduced curve in 25] (where he considered also the higher rank case).

In the case of a family of reducible curves, the (relative) compactification problem is more difficult, as the relative Jacobian itself does not behave well. In fact, the relative Jacobian might not even be a scheme (see Mumford's famous example in [6, p. 210]). Even when it is - for instance, when the irreducible components of the curves in the family are geometrically integral [6, p. 210] - the relative Jacobian might not be either separated or of finite type over the base.

Received by the editors December 15, 1997 and, in revised form, May 2, 2000.

2000 Mathematics Subject Classification. Primary 14H40, 14H60; Secondary 14D20.

Research supported by an MIT Japan Program Starr fellowship, by PRONEX, Convênio 41/96/0883/00 and CNPq, Proc. 300004/95-8. 
Despite the mentioned difficulties, Ishida [19] managed to adapt Oda's and Seshadri's method to describe several compactifications of subspaces of the relative Jacobian, though his hypotheses are numerous.

After a long time without any work on the relative compactification problem, Caporaso 7] showed how to compactify the relative Jacobian over the moduli of Deligne-Mumford stable curves, $\bar{M}_{g}$, by putting on the boundary invertible sheaves on curves derived from stable curves. One year later, Pandharipande 24] produced the same compactification, with the boundary points now representing torsion-free, rank-1 sheaves, as in Seshadri's [25].

Both Caporaso and Pandharipande based their constructions on Gieseker's construction of $\bar{M}_{g}$ [13], and thus their method cannot be extended to an arbitrary family of curves. However, at about the same time Simpson [27, Section 1] constructed moduli spaces of coherent sheaves over any family of projective varieties. The three used GIT.

The main disadvantage of the constructions found so far for reducible curves is the absence of a Poincaré sheaf.

Roughly speaking, the present article aims at constructing a natural relative compactification of the relative Jacobian over a projective, flat family of geometrically reduced and connected curves. In contrast to earlier relative compactifications, ours admits a Poincaré sheaf, after an étale base change. The points of our compactification correspond to simple, torsion-free, rank-1 sheaves that are semi-stable with respect to a given polarization. (Strictly speaking, to obtain separated spaces we have to restrict ourselves to quasi-stable sheaves; the concept of quasi-stability is a new one, introduced in Subsection 1.2 of the present article, and is intermediate between stability and semi-stability.) To compare our relative compactification with that obtained by Seshadri in [25, Part 7], we use theta functions. In contrast with all the past approaches, we do not use GIT. (The method of theta functions has already been used by Faltings to construct the moduli of semi-stable vector bundles on a smooth complete curve (see [12] or [26]).) It must be said that our relative compactification is an algebraic space, rather than a scheme. (It could not be otherwise, as Mumford's example [6, p. 210] shows.) But we show that it becomes a scheme, after an étale base change. The reader will find next a more detailed account of our results.

0.2. Our results. Let $S$ be a locally Noetherian scheme. Let $f: X \rightarrow S$ be a projective, flat map whose geometric fibers are connected, reduced curves. Let

$$
\mathbf{P}^{*}:(\text { Schemes } / S)^{\mathrm{o}} \rightarrow(\text { Sets })
$$

be the relative Jacobian functor, defined on an $S$-scheme $T$ as the set of invertible sheaves on $X \times{ }_{S} T$. Let $\mathbf{P}$ be the étale sheaf associated to $\mathbf{P}^{*}$. Artin [5] showed that the functor $\mathbf{P}$ is represented by an algebraic space $P$, locally of finite type over $S$.

The algebraic space $P$ is formally smooth over $S$. If the geometric fibers of $f$ are integral, then the subspace $P_{d} \subseteq P$, parameterizing invertible sheaves with Euler characteristic $d$, is a separated scheme of finite type over $S$ [15]. In general, however, $P_{d}$ may not even be of finite type over $S$.

In order to compactify $P$ over $S$ in general, it would be natural, following Mayer and Mumford [22], to consider the functor

$$
\mathbf{F}^{*}:(\text { Schemes } / S)^{\circ} \rightarrow(\text { Sets }),
$$


defined on an $S$-scheme $T$ as the set of $T$-flat, coherent sheaves on $X \times{ }_{S} T$ whose fibers over $T$ are torsion-free, rank-1 sheaves. Defining $\mathbf{F}$ as the étale sheafification of $\mathbf{F}^{*}$, it is clear that $\mathbf{F}$ contains $\mathbf{P}$ as an open subfunctor. It is also easy to show that $\mathbf{F}$ "contains enough degenerations". In other words, $\mathbf{F}$ meets the existence condition of the valuative criterion of properness, without necessarily meeting the uniqueness condition. However, the functor $\mathbf{F}$ is not representable by an algebraic space in general, the obstruction being the existence of torsion-free, rank-1 sheaves that are not simple (i.e. admit non-trivial endomorphisms). In fact, considering the subfunctor $\mathbf{J} \subseteq \mathbf{F}$, parameterizing sheaves with simple fibers, it follows from [3, Thm. 7.4, p. 99] that $\mathbf{J}$ is represented by an algebraic space $J$. It is clear that $J$ contains $P$ as an open subspace, since the geometric fibers of $f$ are reduced and connected. But does $J$ "contain enough degenerations" over $S$ ? The surprising answer we obtain in Theorem 32 of the present article is: yes! The upshot is that we do not need to consider all torsion-free, rank-1 sheaves to compactify the relative Jacobian, but just those that are simple. Of course, $J$ is neither separated nor of finite type over $S$ in general, since neither is the open subspace $P$. Nevertheless, since $J$ is a fine moduli space, it is worthwhile to analyze $J$ and, perhaps, obtain from $J$ "coarse moduli spaces" that behave better than $J$. The present article is thus devoted to the study of $J$.

Since $J$ is awkwardly "big", we need to decompose $J$ into simpler "pieces", and for that we use polarizations like those defined by Seshadri in 25, Part 7, p. 153]. As a matter of fact, Seshadri used numerical polarizations but, since we want to deal with a family of curves, we prefer to use "continuous" polarizations. For us, a polarization on $X / S$ is a vector bundle $\mathcal{E}$ on $X$ with rank $r>0$ and relative degree $-r d$ over $S$, for a certain integer $d$. (See Observation 57 for the relationship with Seshadri's polarization.) Of course, there are natural choices of relative polarizations, as the structure sheaf $\mathcal{O}_{X}$ and those constructed from $\mathcal{O}_{X}$ and the relative dualizing sheaf $\omega$, when the fibers of $f$ are Gorenstein. (The latter were used by Pandharipande 24.)

Associated to a polarization $\mathcal{E}$ on $X / S$ we have the usual classes of stable and semi-stable torsion-free, rank-1 sheaves. In Section 1 we define two new classes of sheaves, those of quasi-stable and $\sigma$-quasi-stable torsion-free, rank-1 sheaves (see Subsections 1.2 and 1.4), where $\sigma: S \rightarrow X$ is a section of $f$ through the $S$-smooth locus of $X$. These new classes of sheaves are important for their cohomological and degeneration properties (see Sections 2 and 3 ). Let $J_{\mathcal{E}}^{s}\left(\operatorname{resp} . J_{\mathcal{E}}^{s s}\right.$, resp. $J_{\mathcal{E}}^{q s}$, resp. $J_{\mathcal{E}}^{\sigma}$ ) be the subspace of $J$ parameterizing sheaves with stable (resp. semi-stable, resp. quasi-stable, resp. $\sigma$-quasi-stable fibers) with respect to the polarization $\mathcal{E}$. It follows from the cohomological characterizations of Subsection 2.2 that all the above subspaces are open in $J$. In general, neither $J_{\mathcal{E}}^{q s}$ nor $J_{\mathcal{E}}^{s s}$ is separated over $S$. These spaces are still "too big". Nevertheless, applying the method of Langton's [21] (see Section 3), we prove the following theorem.

Theorem A. The algebraic space $J_{\mathcal{E}}^{s s}$ is of finite type over $S$. In addition,

(1) $J_{\mathcal{E}}^{s s}$ and $J_{\mathcal{E}}^{q s}$ are universally closed over $S$;

(2) $J_{\mathcal{E}}^{s}$ is separated over $S$;

(3) $J_{\mathcal{E}}^{\sigma}$ is proper over $S$.

Proof. See Section 4.

Provided it is convenient to fix a section $\sigma$ of $f$ through the $S$-smooth locus of $X$ (the case of a family of pointed curves), we may restrict ourselves to $J_{\mathcal{E}}^{\sigma}$. We 
can always make an étale base change to obtain enough sections of $f$. In fact, a suitable étale base change will also give us schemes, as stated by the next theorem.

Theorem B. Assume there are sections $\sigma_{1}, \ldots, \sigma_{n}: S \rightarrow X$ of $f$ such that

(1) $\sigma_{i}$ factors through the $S$-smooth locus of $X$ for $i=1, \ldots, n$;

(2) for every $s \in S$, every irreducible component of $X(s)$ is geometrically integral and contains $\sigma_{i}(s)$ for some $i$.

Then, $J$ is a scheme.

Proof. See Subsection 7.1.

However, it might not always be appropriate to change the base scheme, or even to fix a section $\sigma$ of $f$ through the $S$-smooth locus of $X$, assuming one exists. To overcome these problems, we can use theta functions (associated to vector bundles on $X$ ) to construct "approximations" of the algebraic spaces $J_{\mathcal{E}}^{s s}, J_{\mathcal{E}}^{q s}$ and $J_{\mathcal{E}}^{\sigma}$ that are locally projective schemes over $S$, as we describe next. Let $\Sigma \subseteq J_{\mathcal{E}}^{s s}$ be an open subspace, and denote by $\pi: \Sigma \rightarrow S$ the structure map. Then, there is a natural invertible sheaf $\mathcal{L}_{\mathcal{E} \mid \Sigma}$ on $\Sigma$, uniquely defined from the polarization $\mathcal{E}$ (see Subsection 7.1). In addition, there is a natural quasi-coherent graded subsheaf of $\mathcal{O}_{S^{-}}$algebras,

$$
V_{\mathcal{E} \mid \Sigma} \subseteq \Gamma_{\mathcal{E} \mid \Sigma}:=\bigoplus_{m \geq 0} \pi_{*} \mathcal{L}_{\mathcal{E} \mid \Sigma}^{\otimes m},
$$

generated by the so-called theta functions, associated to vector bundles on $X$ (see Subsection 7.2). The homogeneous pieces of $V_{\mathcal{E} \mid \Sigma}$ are coherent (Proposition 56). Let $\bar{\Sigma}_{\mathcal{E}}:=\operatorname{Proj}\left(V_{\mathcal{E} \mid \Sigma}\right)$, and consider the natural rational map $\psi: \Sigma \rightarrow \bar{\Sigma}_{\mathcal{E}}$.

Theorem C. Let $\Sigma \subseteq J_{\mathcal{E}}^{s s}$ be an open subspace. Then, the following statements hold.

(1) If $S$ is excellent, then $\bar{\Sigma}_{\mathcal{E}}$ is locally projective over $S$.

(2) The rational map $\psi: \Sigma \rightarrow \bar{\Sigma}_{\mathcal{E}}$ is defined on $\Sigma$, and is scheme-theoretically dominant.

(3) For every $s \in S$, the fibers of $\psi(s)$ are the Jordan-Hölder equivalence classes of $\Sigma(s)$.

(4) If $\Sigma$ is universally closed over $S$, and $J_{\mathcal{E}}^{s} \subseteq \Sigma$, then the restriction of $\psi$ to $J_{\mathcal{E}}^{s}$ is an open embedding.

Proof. See Subsection 7.2.

Finally, we compare our compactification with Seshadri's [25, Part 7] in the case where $S$ is (the spectrum of) an algebraically closed field $k$ (see Section 8 ). Roughly speaking, we show that, if $X$ has at most ordinary double points for singularities, then any structure theta functions detected in Seshadri's compactification are also detected in ours, and vice-versa. (See Theorem 60 and the discussion before it for a precise statement.)

0.3. Convention. All schemes are assumed to be locally Noetherian. By a vector bundle we mean a locally free sheaf of constant rank. If $t: T \rightarrow S$ is a map of schemes, we call $t$ a $T$-point of $S$. If $T=\operatorname{Spec}(k)$ we say that $t$ is a $k$-point of $S$.

Let $f: X \rightarrow S$ be a map of schemes. We denote by $\mathcal{O}_{X}(1)$ a (relatively) ample (invertible) sheaf on $X / S$, and put $\mathcal{F}(m):=\mathcal{F} \otimes \mathcal{O}_{X}(1)^{\otimes m}$ for every coherent sheaf $\mathcal{F}$ on $X$ and every integer $m$. If $T \rightarrow S$ is a map of schemes, let $f_{T}: X \times{ }_{S} T \rightarrow T$ 
denote the induced map. If $\mathcal{O}_{X}(1)$ is an ample sheaf on $X / S$ and $Y$ is a closed subscheme of $X \times_{S} T$, let $\mathcal{O}_{Y}(1)$ denote the pullback of $\mathcal{O}_{X}(1)$ to $Y$.

Let $X \rightarrow S$ be a projective map of schemes. If $\mathcal{F}$ is an $S$-flat, coherent sheaf on $X$, let $\chi(\mathcal{F} / S)$ denote the relative Euler characteristic of $\mathcal{F}$ over $S$. If, in addition, $X$ is flat over $S$, and $\mathcal{F}$ is a vector bundle of $\operatorname{rank} r$, $\operatorname{let} \operatorname{deg}(\mathcal{F} / S):=\chi(\mathcal{F} / S)-r \chi\left(\mathcal{O}_{X} / S\right)$. By flatness, $\chi(\mathcal{F} / S)$ and $\operatorname{deg}(\mathcal{F} / S)$ are locally constant on $S$. If $T \rightarrow S$ is a map of schemes and $\mathcal{F}$ is a coherent sheaf on $X$, let $\mathcal{F} \otimes \mathcal{O}_{T}$ or $\mathcal{F}_{T}$ denote the pullback of $\mathcal{F}$ to $X \times{ }_{S} T$. If $t$ is a $k$-point of $T$ lying over $s \in S$, let $X(t)$ denote the extension of $X(s)$ over $k$, and $\mathcal{F}(t)$ the pullback of $\mathcal{F}$ to $X(t)$. If $s \in S$ and $k \supseteq k(s)$ is a field extension, let $X(k)$ denote the extension of $X(s)$ over $k$, and $\mathcal{F}(k)$ the pullback of $\mathcal{F}$ to $X(k)$.

Whenever the base scheme $S$ is clear, we let $X \times T$ or $X_{T}$ denote the fibered product of two $S$-schemes $X$ and $T$.

\section{Semi-Stable Sheaves}

1.1. Torsion-free, rank-1 sheaves. Let $X$ be a geometrically reduced, projective scheme of pure dimension 1 over a field $k$. We say that $X$ is a curve. Let $X_{1}, \ldots, X_{n}$ denote the irreducible components of $X$. Assume throughout Section 1 that $X_{1}, \ldots, X_{n}$ are geometrically integral. This is a mild assumption, as there is always a finite and separable field extension $k^{\prime} \supseteq k$ such that the irreducible components of $X\left(k^{\prime}\right)$ are geometrically integral (see Lemma 18).

A subcurve of $X$ is a reduced closed subscheme $Y \subseteq X$ of pure dimension 1 . The empty set will also be considered a subcurve of $X$. If $Y, Z \subseteq X$ are subcurves, let $Y \wedge Z$ denote the maximum subcurve of $X$ contained in $Y \cap Z$, and $Y-Z$ the minimum subcurve containing $Y \backslash Z$. If $Y \subseteq X$ is a subcurve, let $Y^{c}:=X-Y$.

Let $I$ be a coherent sheaf on $X$. We say that $I$ is torsion-free if $I$ has no embedded components. We say that $I$ is rank-1 if $I$ has generic rank 1 at every irreducible component of $X$. We say that $I$ is simple if $\operatorname{End}_{X}(I)=k$. If $I$ is invertible, then $I$ is torsion-free, rank-1, and also simple if $X$ is connected.

Let $I$ be a torsion-free, rank-1 sheaf on $X$. If $Y \subseteq X$ is a subcurve, denote by $I_{Y}$ the maximum torsion-free quotient of $\left.I\right|_{Y}$. Of course, there is a canonical surjection $I \rightarrow I_{Y}$. We may understand $I_{Y}$ as the unique quotient of $I$ that has support $Y$ and is torsion-free there. We say that $I$ is decomposable if there are proper subcurves $Y, Z \varsubsetneqq X$ such that the canonical injection $I \rightarrow I_{Y} \oplus I_{Z}$ is an isomorphism. If this is the case, we say that $I$ decomposes at $Y$ (or $Z$ ).

Proposition 1. Let I be a torsion-free, rank-1 sheaf on $X$. Then, I is simple if and only if $I$ is not decomposable.

Proof. Clearly, if $I$ is decomposable, then $I$ is not simple. Assume $I$ is not simple. Then, there is an endomorphism $h: I \rightarrow I$ that is not a multiple of the identity. Let $Y \subseteq X$ be the subcurve such that $I_{Y} \cong \operatorname{im}(h)$, and let $h^{\prime}: I_{Y} \hookrightarrow I$ denote the induced injection. Since $h \neq 0$, the subcurve $Y$ is not empty. Since $I_{W}$ is simple for every irreducible component $W \subseteq X$ by [3, Lemma 5.4, p. 83], up to subtracting a multiple of the identity from $h$, we may assume $Y \neq X$. The map $h^{\prime}$ factors through $J:=\operatorname{ker}\left(I \rightarrow I_{Z}\right)$, where $Z:=Y^{c}$. Moreover, since $h^{\prime}$ and the composition $J \hookrightarrow I \rightarrow I_{Y}$ are injective, $h^{\prime}$ is actually an isomorphism onto $J$. So, $\chi(I)=\chi\left(I_{Y}\right)+\chi\left(I_{Z}\right)$, and hence $I=I_{Y} \oplus I_{Z}$. 
The above proposition does not hold in higher rank, even if we assume $X$ is smooth. In fact, if $X$ is smooth and not rational, then any vector bundle $E$ fitting in the middle of a non-split short exact sequence of the form

$$
0 \rightarrow \mathcal{O}_{X} \rightarrow E \rightarrow \mathcal{O}_{X} \rightarrow 0
$$

is neither simple nor decomposable. Though easy to state and prove, Proposition 1 is the key reason why we are able to get fine moduli spaces in the rank-1 case.

The following lemma is useful for determining when a torsion-free, rank-1 sheaf is simple (cf. Example 5).

Lemma 2. Let $Y, Z \subseteq X$ be non-empty subcurves covering $X$. Let $M$ be a torsionfree, rank-1 sheaf on $X$. Then, the following statements hold.

(1) If $Y \wedge Z \neq \emptyset$, and both $M_{Y}$ and $M_{Z}$ are simple, then $M$ is simple.

(2) If there is an exact sequence of the form

$$
0 \rightarrow I \rightarrow M \rightarrow J \rightarrow 0
$$

where I (resp. J) is a simple, torsion-free, rank-1 sheaf on $Y$ (resp. Z), then $M$ is simple if and only if the sequence is not split.

Proof. Assume there are subcurves $X_{1}, X_{2} \subseteq X$ such that $M=M_{X_{1}} \oplus M_{X_{2}}$. In addition, assume there is a surjection $\mu: M \rightarrow J$, where $J$ is a simple, torsion-free, rank-1 sheaf on a subcurve $Z \subseteq X$. Of course, $\mu$ is the direct sum of two maps, $\mu_{1}: M_{X_{1}} \rightarrow J$ and $\mu_{2}: M_{X_{2}} \rightarrow J$. Since $\mu$ is surjective, $\operatorname{im}\left(\mu_{i}\right)=M_{Z_{i}}$, where $Z_{i}:=Z \wedge X_{i}$ for $i=1,2$. So, $J=M_{Z_{1}} \oplus M_{Z_{2}}$. Since $J$ is simple, either $Z \subseteq X_{1}$ or $Z \subseteq X_{2}$.

We prove (1) now. Assume $M=M_{X_{1}} \oplus M_{X_{2}}$ for subcurves $X_{1}, X_{2} \subseteq X$. Apply the above reasoning twice, to both $J:=M_{Y}$ and $J:=M_{Z}$. Without loss of generality, either $Y \subseteq X_{1}$ and $Z \subseteq X_{2}$, or $Y \cup Z \subseteq X_{1}$. By hypothesis, $Y \wedge Z \neq \emptyset$. So, $Y \cup Z \subseteq X_{1}$. Since $Y \cup Z=X$, we have $X_{1}=X$. By Proposition 1 , the sheaf $M$ is simple.

We prove $(2)$ now. The $(\Rightarrow)$ part is trivial. We show $(\Leftarrow)$ now. Assume by contradiction that $M=M_{X_{1}} \oplus M_{X_{2}}$ for proper subcurves $X_{1}, X_{2} \varsubsetneqq X$. Apply the reasoning in the first paragraph of the proof to the surjection $\mu: M \rightarrow J$. Without loss of generality, we may assume $Z \subseteq X_{1}$. So, $\mu_{2}=0$, and hence $I=\operatorname{ker}\left(\mu_{1}\right) \oplus M_{X_{2}}$. Since $I$ is simple and $X_{2}$ is not empty, $\operatorname{ker}\left(\mu_{1}\right)=0$. So, $J=M_{X_{1}}$, and thus the sequence in (2) is split, a contradiction.

1.2. Semi-stable sheaves. Let $d$ be an integer, and $E$ a vector bundle on $X$ of rank $r>0$ and degree $-r d$. We say that $E$ is a polarization on $X$. For every subcurve $Y \subseteq X$, let $e_{Y}:=-\left.\operatorname{deg} E\right|_{Y}$. Note that $\left.E\right|_{Y}$ is a polarization on a non-empty subcurve $Y \subseteq X$ if $r \mid e_{Y}$.

Observe that, if $I$ is a torsion-free, rank-1 sheaf on $X$, and $F$ is a vector bundle on $X$ of rank $m$ and degree $f$, then $\chi(I \otimes F)=m \chi(I)+f$.

Let $I$ be a torsion-free, rank-1 sheaf on $X$ with $\chi(I)=d$. By our observation above, $\chi(I \otimes E)=0$. We say that $I$ is stable (resp. semi-stable) with respect to $E$ if, for every non-empty, proper subcurve $Y \varsubsetneqq X$,

$$
\chi\left(I_{Y}\right)>e_{Y} / r\left(\operatorname{resp} . \chi\left(I_{Y}\right) \geq e_{Y} / r\right),
$$


or equivalently,

$$
\chi\left(I_{Y} \otimes E\right)>0\left(\text { resp. } \chi\left(I_{Y} \otimes E\right) \geq 0\right) .
$$

If $X$ is irreducible, then any torsion-free, rank-1 sheaf $I$ on $X$ with $\chi(I)=d$ is stable with respect to $E$. When the choice of polarization is clear, we will not make reference to it.

Strictly speaking, the notions of stability and semi-stability depend rather on the multi-slope $\underline{\mu}_{E}:=-\left(e_{X_{1}}, \ldots, e_{X_{n}}\right) / r$ of $E$. So, it is not difficult to show that our notions of polarization, stability and semi-stability are equivalent to Seshadri's (Observation 57). However, in dealing with families of curves we will find it more convenient to think of $E$ as the polarization, rather than $\mu_{E}$. In addition, $E$ gives us a line bundle on the moduli space of torsion-free, rank-1 sheaves of Euler characteristic $d$ (see Subsection 6.2).

Let $I$ be a torsion-free, rank-1 sheaf on $X$ with $\chi(I)=d$. For every subcurve $Y \subseteq X$, let $\beta_{I}(Y):=\chi\left(I_{Y}\right)-e_{Y} / r$. Of course, $I$ is stable (resp. semi-stable) if and only if $\beta_{I}(Y)>0$ (resp. $\beta_{I}(Y) \geq 0$ ) for every non-empty, proper subcurve $Y \varsubsetneqq X$. If $I$ is semi-stable and $Y \subseteq X$ is a non-empty subcurve, then $\beta_{I}(Y)=0$ if and only if $I_{Y}$ is semi-stable with respect to $\left.E\right|_{Y}$.

For a fixed $I$, the $\beta_{I}(Y)$ measure how distant $I$ is from being stable or semistable. There are relations among the $\beta_{I}(Y)$, as stated below.

Lemma 3. Let $I$ be a torsion-free, rank-1 sheaf on $X$ with $\chi(I)=d$. If $Y, Z \subseteq X$ are subcurves, then

$$
\chi\left(I_{Y \cup Z}\right)+\chi\left(I_{Y \wedge Z}\right) \leq \chi\left(I_{Y}\right)+\chi\left(I_{Z}\right),
$$

or equivalently,

$$
\beta_{I}(Y \cup Z)+\beta_{I}(Y \wedge Z) \leq \beta_{I}(Y)+\beta_{I}(Z) .
$$

Proof. Form the commutative diagram

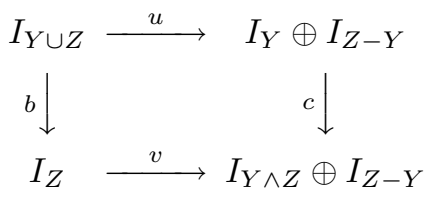

where the maps are (sums of) the natural restriction maps. Note that $b$ and $c$ are surjective, whereas $u$ and $v$ are injective with finite-length cokernels. Hence, coker $(u)$ maps onto $\operatorname{coker}(v)$ and, comparing their Euler characteristics,

$$
\chi\left(I_{Y}\right)+\chi\left(I_{Z-Y}\right)-\chi\left(I_{Y \cup Z}\right) \geq \chi\left(I_{Y \wedge Z}\right)+\chi\left(I_{Z-Y}\right)-\chi\left(I_{Z}\right) .
$$

Let $W \subseteq X$ be an irreducible component and $I$ a semi-stable sheaf on $X$. It follows from Lemma 3 that there is a minimum subcurve $Z \subseteq X$ containing $W$ with $\beta_{I}(Z)=0$. We say that $I$ is $W$-quasi-stable with respect to $E$ if $\beta_{I}(Y)>0$ for all proper subcurves $Y \varsubsetneqq X$ containing $W$. A semi-stable sheaf $I$ on $X$ is stable if and only if $I$ is $W$-quasi-stable for every irreducible component $W \subseteq X$.

There is yet another interesting class of sheaves, this time independent of the choice of an irreducible component. We say that a semi-stable sheaf $I$ on $X$ is quasistable with respect to $E$ if there is an irreducible component $W \subseteq X$ such that $I$ is 
$W$-quasi-stable with respect to $E$. Note that a quasi-stable sheaf is simple, an easy corollary of Proposition 1.

The notion of $W$-quasi-stability is not so easy to manage when dealing with families of curves. We shall often replace it with the equivalent, but more suitable, notion of $p$-quasi-stability. Let $p \in X$ be a non-singular point. We say that a semistable sheaf $I$ on $X$ is $p$-quasi-stable with respect to $E$ if $\beta_{I}(Y)>0$ for all proper subcurves $Y \varsubsetneqq X$ containing $p$.

A semi-stable sheaf $I$ on $X$ is $X_{i}$-quasi-stable (for $i=1, \ldots, n$ ) if and only if $I$ is $\left(r \underline{\delta}_{i}\right)$-quasi-stable (see [10, Section 4]), where $\underline{\delta}_{i}$ is the $n$-uple whose only non-zero component is the $i$-th component with value 1 .

We shall see in Sections 2 and 3 that the notion of quasi-stability is natural and useful.

Lemma 4. Let $Y, Z \varsubsetneqq X$ be proper subcurves covering $X$ such that $Y \wedge Z=\emptyset$, but $Y \cap Z \neq \emptyset$. Let $I$ (resp. J) be a torsion-free, rank-1 sheaf on $Y$ (resp. Z). Then, there is a non-split exact sequence of the form

$$
0 \rightarrow J \rightarrow M \rightarrow I \rightarrow 0
$$

Proof. We need to show that $\operatorname{Ext}_{X}^{1}(I, J) \neq 0$. Since $I$ and $J$ are torsion-free sheaves supported on $Y$ and $Z$, and $Y \wedge Z=\emptyset$, we have $\underline{\operatorname{Hom}}_{X}(I, J)=0$. Thus,

$$
\operatorname{Ext}_{X}^{1}(I, J)=H^{0}\left(X, \underline{\operatorname{Ext}}_{X}^{1}(I, J)\right) .
$$

Of course, the topological support of $\operatorname{Ext}_{X}^{1}(I, J)$ is contained in $Y \cap Z$. Since $Y \cap Z \neq \emptyset$, there is $p \in Y \cap Z$. Let $\mathcal{O}_{p}$ denote the local ring of $X$ at $p$, with maximal ideal $\mathcal{M}_{p}$. We need only show that $\operatorname{Ext}_{\mathcal{O}_{p}}^{1}\left(I_{p}, J_{p}\right) \neq 0$. Let $\mathcal{M}_{Y} \subseteq \mathcal{O}_{p}$ (resp. $\mathcal{M}_{Z} \subseteq \mathcal{O}_{p}$ ) be the ideal of $Y$ (resp. $Z$ ) at $p$. By hypothesis, $\mathcal{M}_{Y} \cap \mathcal{M}_{Z}=0$ and $\mathcal{M}_{Y}+\mathcal{M}_{Z}$ is a primary ideal of $\mathcal{M}_{p}$.

Let

$$
\left(\frac{\mathcal{O}_{p}}{\mathcal{M}_{Y}}\right)^{\oplus s_{1}} \stackrel{\phi}{\rightarrow}\left(\frac{\mathcal{O}_{p}}{\mathcal{M}_{Y}}\right)^{\oplus s_{0}} \rightarrow I_{p} \rightarrow 0
$$

be a presentation of $I_{p}$. Applying $\operatorname{Ext}_{\mathcal{O}_{p}}^{1}\left(-, J_{p}\right)$ to $(4.1)$, we obtain a sequence

$$
0 \rightarrow \operatorname{Ext}_{\mathcal{O}_{p}}^{1}\left(I_{p}, J_{p}\right) \rightarrow H^{\oplus s_{0}} \stackrel{\phi^{*} \otimes H}{\longrightarrow} H^{\oplus s_{1}},
$$

where

$$
H:=\operatorname{Ext}_{\mathcal{O}_{p}}^{1}\left(\frac{\mathcal{O}_{p}}{\mathcal{M}_{Y}}, J_{p}\right)
$$

and $\phi^{*}$ is the dual of $\phi$. The sequence (4.2) is exact, since $\operatorname{Hom}_{\mathcal{O}_{p}}\left(K, J_{p}\right)=0$ for all $\mathcal{O}_{p}$-modules $K$ with $\mathcal{M}_{Y} K=0$. So, we need only show that $\phi^{*} \otimes H$ is not injective.

Assume by contradiction that $\phi^{*} \otimes H$ is injective. Since $H$ has finite length, it follows by a standard argument that $\phi^{*} \otimes k(p)$ is injective. Since $\phi^{*}$ is a map of free modules over the local ring $\mathcal{O}_{p} / \mathcal{M}_{Y}$, it follows that $\phi^{*}$ is injective. Since (4.1) is exact, $\operatorname{Hom}_{\mathcal{O}_{p}}\left(I_{p}, \mathcal{O}_{p} / \mathcal{M}_{Y}\right)=0$. Since $I$ is torsion-free, rank-1 on $Y$, we get $I_{p}=0$, a contradiction.

The above lemma allows us to construct torsion-free, rank-1 sheaves on $X$ with prescribed Jordan-Hölder filtrations, as we shall see in Theorem 7. 
Example 5. If $X$ is reducible, then there are semi-stable sheaves that are not simple. If $X$ has only two irreducible components, then every simple, semi-stable sheaf is quasi-stable. If $X$ has more than two components, then there might be simple, semi-stable sheaves that are not quasi-stable. For instance, suppose that there are connected subcurves $X_{1}, X_{2}, X_{3} \subseteq X$ covering $X$ such that $X_{i} \wedge X_{j}=\emptyset$ for $i \neq j$, but $X_{1} \cap X_{2} \neq \emptyset$ and $X_{1} \cap X_{3} \neq \emptyset$. Let $I_{1}, I_{2}, I_{3}$ be semi-stable, simple sheaves on $X_{1}, X_{2}, X_{3}$, respectively. By Lemma 4 , since $X_{1} \cap X_{2} \neq \emptyset$ and $X_{1} \cap X_{3} \neq \emptyset$, there is a non-split exact sequence of the form

$$
0 \rightarrow I_{2} \oplus I_{3} \rightarrow I \rightarrow I_{1} \rightarrow 0
$$

whose push-out to $I_{2}$ (resp. $I_{3}$ ) is a non-split exact sequence of the form

$$
0 \rightarrow I_{2} \rightarrow I_{X_{1} \cup X_{2}} \rightarrow I_{1} \rightarrow 0 \quad\left(\text { resp. } 0 \rightarrow I_{3} \rightarrow I_{X_{1} \cup X_{3}} \rightarrow I_{1} \rightarrow 0\right. \text { ). }
$$

Since $I_{1}, I_{2}, I_{3}$ are semi-stable, so are $I, I_{X_{1} \cup X_{2}}$ and $I_{X_{1} \cup X_{3}}$. Thus, $I$ is not quasistable. In addition, since the latter exact sequences are not split, $I_{X_{1} \cup X_{2}}$ and $I_{X_{1} \cup X_{3}}$ are simple by Lemma 2, (2). So, $I$ is simple by Lemma 2, (1). We have thus produced a simple, semi-stable sheaf $I$ that is not quasi-stable.

1.3. Jordan-Hölder filtrations. Let $E$ be a polarization on $X$. Let $I$ be a semistable sheaf on $X$ with respect to $E$. We describe now a filtration of $I$. To start with, let $I_{0}:=I$ and $Z_{0}:=X$. Let $Y_{0} \subseteq X$ be a non-empty subcurve such that $I_{Y_{0}}$ is stable with respect to $\left.E\right|_{Y_{0}}$. Let $I_{1}:=\operatorname{ker}\left(I \rightarrow I_{Y_{0}}\right)$. Clearly, the sheaf $I_{1}$ is torsion-free, rank-1 on $Z_{1}:=Y_{0}^{c}$, and semi-stable with respect to $\left.E\right|_{Z_{1}}$, if not zero. Repeating the above procedure with $I_{1}$, in place of $I$, and so on, we end up with filtrations

$$
\left\{\begin{array}{l}
0=I_{q+1} \varsubsetneqq I_{q} \varsubsetneqq \cdots \varsubsetneqq I_{1} \varsubsetneqq I_{0}=I, \\
\emptyset=Z_{q+1} \varsubsetneqq Z_{q} \varsubsetneqq \cdots \varsubsetneqq Z_{1} \varsubsetneqq Z_{0}=X
\end{array}\right.
$$

with the following properties:

(1) for $i=0, \ldots, q$, the sheaf $I_{i}$ is torsion-free, rank-1 on the subcurve $Z_{i} \subseteq X$, and is semi-stable with respect to $\left.E\right|_{Z_{i}}$,

(2) for $i=0, \ldots, q$, the quotient $I_{i} / I_{i+1}$ is torsion-free, rank-1 on the subcurve $Y_{i}:=Z_{i}-Z_{i+1}$, and is stable with respect to $\left.E\right|_{Y_{i}}$.

We call the above filtration of $I$ a Jordan-Hölder filtration. A Jordan-Hölder filtration depends on the choices made in its construction, but

$$
\operatorname{Gr}(I):=I_{0} / I_{1} \oplus I_{1} / I_{2} \oplus \cdots \oplus I_{q} / I_{q+1}
$$

depends only on $I$ by the Jordan-Hölder theorem. In particular, the collection of subcurves $\left\{Y_{0}, \ldots, Y_{q}\right\}$ covering $X$ depends only on $I$. It is clear that $\operatorname{Gr}(I)$ is torsion-free, rank-1, and semi-stable with respect to $E$. In addition, we have $\operatorname{Gr}(\operatorname{Gr}(I))=\operatorname{Gr}(I)$. If $I$ is stable, then $\operatorname{Gr}(I)=I$. We say that two semi-stable sheaves, $I$ and $J$, are Jordan-Hölder equivalent (or $J H$-equivalent) if $\operatorname{Gr}(I) \cong \operatorname{Gr}(J)$.

Proposition 6. Let I be a semi-stable sheaf on X. Let

$$
\left\{\begin{array}{l}
0=I_{q+1} \varsubsetneqq I_{q} \varsubsetneqq \cdots \varsubsetneqq I_{1} \varsubsetneqq I_{0}=I, \\
\emptyset=Z_{q+1} \varsubsetneqq Z_{q} \varsubsetneqq \cdots \varsubsetneqq Z_{1} \varsubsetneqq Z_{0}=X
\end{array}\right.
$$

be a Jordan-Hölder filtration of $I$. Let $W \subseteq X$ be an irreducible component. Then, the following statements are equivalent. 
(1) $I$ is $W$-quasi-stable.

(2) $I_{i}$ is $W$-quasi-stable for $i=0, \ldots, q$.

(3) $W \subseteq Z_{q}$, and the short exact sequence

$$
0 \rightarrow I_{i+1} \rightarrow I_{i} \rightarrow \frac{I_{i}}{I_{i+1}} \rightarrow 0
$$

is not split for $i=0, \ldots, q-1$.

Proof. See [10, Prop. 6].

The following theorem shows that all Jordan-Hölder equivalence classes have $W$-quasi-stable representatives for every irreducible component $W \subseteq X$.

Theorem 7. Assume $X$ is connected. Let $Y_{0}, \ldots, Y_{q} \subseteq X$ be subcurves covering $X$ with $Y_{i} \wedge Y_{j}=\emptyset$ for $i \neq j$, and $J_{0}, \ldots, J_{q}$ stable sheaves on $Y_{0}, \ldots, Y_{q}$, respectively. Let $W \subseteq X$ be an irreducible component. Then, there is a $W$-quasi-stable sheaf $I$ on $X$ such that $\operatorname{Gr}(I) \cong J_{0} \oplus \cdots \oplus J_{q}$.

Proof. Without loss of generality, we may assume $W \subseteq Y_{q}$. Since $X$ is connected, we may also assume $\left(Y_{q} \cup \cdots \cup Y_{i}\right) \cap Y_{i-1} \neq \emptyset$ for $i=1, \ldots, q$. Let $Z_{i}:=Y_{i} \cup \cdots \cup Y_{q}$ for $i=0, \ldots, q$. Let $I_{q}:=J_{q}$. We construct recursively a torsion-free, rank-1 sheaf $I_{i}$ on $Z_{i}$ for $i=q-1, \ldots, 0$ as follows: assume we are given $I_{q}, \ldots, I_{i}$ for a certain $i \in\{1, \ldots, q\}$; then, let $I_{i-1}$ be the middle sheaf in a non-split exact sequence of the form

$$
0 \rightarrow I_{i} \rightarrow I_{i-1} \rightarrow J_{i-1} \rightarrow 0
$$

whose existence is guaranteed by Lemma 4 . Let $I:=I_{0}$. We have a Jordan-Hölder filtration

$$
\left\{\begin{array}{l}
0=: I_{q+1} \varsubsetneqq I_{q} \varsubsetneqq \cdots \varsubsetneqq I_{1} \varsubsetneqq I_{0}=I, \\
\emptyset=: Z_{q+1} \varsubsetneqq Z_{q} \varsubsetneqq \cdots \varsubsetneqq Z_{1} \varsubsetneqq Z_{0}=X
\end{array}\right.
$$

of $I$ such that $\operatorname{Gr}(I)=J_{0} \oplus \cdots \oplus J_{q}$. Moreover, $I$ is $W$-quasi-stable by Proposition 6 .

1.4. Families. Let $f: X \rightarrow S$ be a flat, projective map whose geometric fibers are curves. We say that $X / S$ is a family of curves. Let $\mathcal{I}$ be an $S$-flat coherent sheaf on $X$. We say that $\mathcal{I}$ is torsion-free (resp. rank-1, resp. simple) on $X / S$ if $\mathcal{I}(s)$ is torsion-free (resp. rank-1, resp. simple) for every geometric point $s$ of $S$.

Let $\mathcal{E}$ be a vector bundle on $X$ of rank $r>0$ such that $r \mid \operatorname{deg}(\mathcal{E} / S)$. We say that $\mathcal{E}$ is a polarization on $X / S$. A torsion-free, rank-1 sheaf $\mathcal{I}$ on $X / S$ is stable (resp. semi-stable, resp. quasi-stable) with respect to $\mathcal{E}$ over $S$ if $\mathcal{I}(s)$ is stable (resp. semi-stable, resp. quasi-stable) with respect to $\mathcal{E}(s)$ for every geometric point $s$ of $S$. Let $\sigma: S \rightarrow X$ be a section of $f$ through the $S$-smooth locus of $X$. A torsionfree, rank-1 sheaf $\mathcal{I}$ on $X / S$ is $\sigma$-quasi-stable with respect to $\mathcal{E}$ over $S$ if $\mathcal{I}(s)$ is $\sigma(s)$-quasi-stable with respect to $\mathcal{E}(s)$ for every geometric point $s$ of $S$.

\section{Cohomological Characterizations}

2.1. Curves. Let $X$ be a curve over a field $k$. Assume that the irreducible components of $X$ are geometrically integral. Let $\omega$ be the dualizing sheaf on $X$. Recall that $\omega$ is simple, torsion-free and rank-1. Let $P$ denote the Jacobian of $X$ 
(see Subsection 0.2). Fix an integer $d$. Let $E$ be a vector bundle on $X$ of rank $r$ and degree $-r d$.

Let $S$ be a $k$-scheme of finite type and $\mathcal{F}$ an $S$-flat, coherent sheaf on $X \times S$. If the Kodaira-Spencer map

$$
\delta_{s}: T_{S, s} \rightarrow \operatorname{Ext}_{X(s)}^{1}(\mathcal{F}(s), \mathcal{F}(s))
$$

of $\mathcal{F}$ at $s$ is surjective for every $s \in S$, then we say that $\mathcal{F}$ is complete over $S$, or $S$-complete.

If $\mathcal{F}$ is $S$-complete and $k^{\prime} \supseteq k$ is a field extension, then $\mathcal{F}\left(k^{\prime}\right)$ is $S\left(k^{\prime}\right)$-complete.

If $\mathcal{F}$ is a vector bundle on $X \times S$ that is complete over $S$, then so is its restriction to $Y \times S$ for every subcurve $Y \subseteq X$. In addition, the determinant map $\pi_{\mathcal{F}}: S \rightarrow P$, sending $s \in S$ to the point of $P$ representing $\operatorname{det} \mathcal{F}(s)$, is surjective on tangent spaces. In particular, if $S$ is $k$-smooth, then $\pi_{\mathcal{F}}$ is smooth.

Lemma 8. For any vector bundle $F$ on $X$, there are a smooth $k$-scheme $S$, an $S$-complete vector bundle $\mathcal{F}$ on $X \times S$ and a $k$-point $s \in S$ with $\mathcal{F}(s) \cong F$.

Proof. The following proof is an expanded version of the rather sketchy argument laid out by Faltings in [12, p. 514].

Fix an ample invertible sheaf $\mathcal{O}_{X}(1)$ on $X$. Let $r$ be the rank of $F$, and $m$ an integer such that $F$ is $m$-regular. Let $h:=h^{0}(X, F(m))$ and $R:=\mathcal{O}_{X}(-m)^{\oplus h}$. Since $F(m)$ is generated by global sections, we obtain a surjection $\rho: R \rightarrow F$ by choosing a basis of $H^{0}(X, F(m))$. Let $Q:=$ Quot $_{R \mid X}$ be the quot scheme, and $s \in Q$ the $k$-point representing $\rho$. Let $\mathcal{G}$ denote the universal quotient on $X \times Q$.

The tangent space $T_{Q, t}$ at a point $t \in Q$ representing a quotient $q: R(t) \rightarrow G$ on $X(t)$ is naturally isomorphic to $\operatorname{Hom}(I, G)$, where $I:=\operatorname{ker}(q)$. The KodairaSpencer map $\delta_{t}$ of $\mathcal{G}$ at $t$ is the coboundary map in the exact sequence

$$
\operatorname{Hom}(I, G) \stackrel{\delta_{t}}{\longrightarrow} \operatorname{Ext}^{1}(G, G) \rightarrow \operatorname{Ext}^{1}(R(t), G)
$$

derived from the short exact sequence

$$
0 \rightarrow I \rightarrow R(t) \stackrel{q}{\rightarrow} G \rightarrow 0
$$

Let $S \subseteq Q$ be the open subscheme parameterizing quotients $q: R \rightarrow G$ where $G$ is a vector bundle of rank $r$ and $m$-regular. Let $\mathcal{F}$ be the restriction of $\mathcal{G}$ to $X \times S$. Of course, $s \in S$. If $t \in S$ is represented by $q: R(t) \rightarrow G$, then

$$
\operatorname{Ext}^{1}(R(t), G)=H^{1}(X(t), G(m))^{\oplus h}=0 .
$$

Since (8.1) is exact, $\delta_{t}$ is surjective.

We show now that $S$ is $k$-smooth. If $t \in Q$ represents a quotient $q: R(t) \rightarrow G$, then the obstruction to smoothness of $Q$ at $t$ lies in a vector space $V$ that is part of an exact sequence of the form

$$
0 \rightarrow H^{1}(X(t), \underline{\operatorname{Hom}}(I, G)) \rightarrow V \rightarrow H^{0}\left(X(t), \underline{\operatorname{Ext}}^{1}(I, G)\right),
$$

where $I:=\operatorname{ker}(q)$. If $t \in S$, then $I$ is a vector bundle, and thus $\underline{\operatorname{Ext}}^{1}(I, G)=0$. Moreover, since $X$ is a curve, the natural map

$$
H^{1}(X(t), \underline{\operatorname{Hom}}(R(t), G)) \rightarrow H^{1}(X(t), \underline{\operatorname{Hom}}(I, G))
$$

is surjective. But $\underline{\operatorname{Hom}}(R(t), G)=G(m)^{\oplus h}$ has trivial first cohomology, since $G$ is $m$-regular. Thus $V=0$, implying that $S$ is smooth at $t$. 
The proof of the following lemma is based on the same ideas used in the proof of [12] Thm. 1.2, p. 514] (see also the proof of [26. Lemma 3.1, p. 166]). However, in our situation the proof is considerably shorter.

Lemma 9. Let $I$ be a torsion-free, rank-1 sheaf on $X$. Let $S$ be an integral $k$ scheme of finite type, and $\mathcal{F}$ an $S$-complete vector bundle on $X \times S$ such that $\chi(I(s) \otimes \mathcal{F}(s)) \leq 0$ and

$$
\chi(I(s) \otimes \mathcal{F}(s)) \leq \chi\left(I_{Y}(s) \otimes \mathcal{F}(s)\right)
$$

for every subcurve $Y \subseteq X$ and every $s \in S$. Then, there are a dense, open subset $U \subseteq S$ and a subcurve $Y \subseteq X$ such that, for every $s \in U$,

(1) $h^{0}(X(s), I(s) \otimes \mathcal{F}(s))=h^{0}\left(X(s), I_{Y}(s) \otimes \mathcal{F}(s)\right)=0$,

(2) all maps $I(s) \rightarrow \mathcal{F}^{*}(s) \otimes \omega(s)$ factor through $I_{Y}(s)$, and every other subcurve of $X$ with this property must contain $Y$.

Proof. By semicontinuity, replacing $S$ by an open dense subscheme, we may assume $h^{0}(X(s), I(s) \otimes \mathcal{F}(s))$ is constant for $s \in S$. For each $s \in S$, let $Y_{s} \subseteq X$ be the minimum subcurve with the property that all maps $I(s) \rightarrow \mathcal{F}^{*}(s) \otimes \boldsymbol{\omega}(s)$ factor through $I_{Y_{s}}(s)$. Replacing $S$ by an open dense subscheme, we may assume $Y_{s}$ is constant for $s \in S$. So, let $Y:=Y_{s}$ for some (and every) $s \in S$.

Note that

$$
\operatorname{Hom}\left(I(s), \mathcal{F}^{*}(s) \otimes \boldsymbol{\omega}(s)\right)=\operatorname{Hom}\left(I_{Y}(s), \mathcal{F}^{*}(s) \otimes \boldsymbol{\omega}(s)\right)
$$

for every $s \in S$. In particular, $h^{0}\left(X(s), I_{Y}(s) \otimes \mathcal{F}(s)\right)$ is constant for $s \in S$. Since $S$ is reduced, the formation of $p_{2 *}\left(p_{1}^{*} I_{Y} \otimes \mathcal{F}\right)$ commutes with base change, where $p_{1}, p_{2}$ are the projections of $X \times S$ onto $X$ and $S$, respectively.

Let $s \in S$. A tangent vector of $S$ at $s$ corresponds to a map $T \rightarrow S$ with image $s$, where $T:=\operatorname{Spec}\left(k(s)[\epsilon] /\left(\epsilon^{2}\right)\right)$. So, it induces a linear map

$$
H^{0}\left(X(s), I_{Y}(s) \otimes \mathcal{F}(s)\right) \rightarrow H^{1}\left(X(s), I_{Y}(s) \otimes \mathcal{F}(s)\right),
$$

obtained as the coboundary map in cohomology associated to the natural short exact sequence

$$
0 \rightarrow I_{Y}(s) \otimes \mathcal{F}(s) \rightarrow\left(p_{1}^{*} I_{Y} \otimes \mathcal{F}\right) \otimes \mathcal{O}_{T} \rightarrow I_{Y}(s) \otimes \mathcal{F}(s) \rightarrow 0 .
$$

Combining the above maps for all tangent vectors of $S$ at $s$, we get a bilinear map

$$
\eta_{s}: T_{S, s} \times H^{0}\left(X(s), I_{Y}(s) \otimes \mathcal{F}(s)\right) \rightarrow H^{1}\left(X(s), I_{Y}(s) \otimes \mathcal{F}(s)\right) .
$$

Since the formation of $p_{2 *}\left(p_{1}^{*} I_{Y} \otimes \mathcal{F}\right)$ commutes with base change, $\eta_{s}=0$. Since $I_{Y}(s)$ is the pullback of $I_{Y}$ for every $s \in S$, the natural diagram of maps

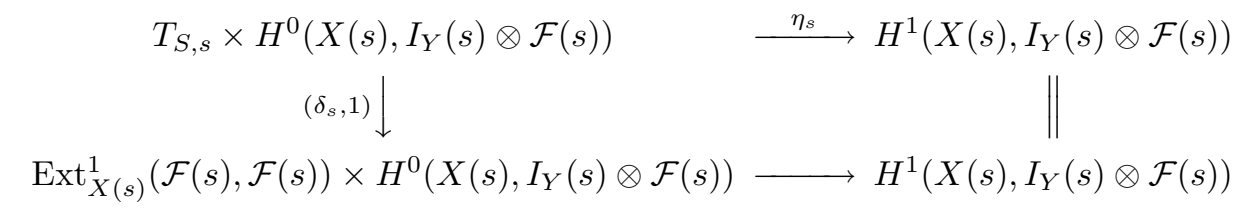

is commutative. Since $\delta_{s}$ is surjective and $\eta_{s}=0$, the bottom map is zero. By Serre duality, the corresponding bilinear map

$\operatorname{Hom}\left(\mathcal{F}(s)^{*}, I_{Y}(s)\right) \times \operatorname{Hom}\left(I_{Y}(s), \mathcal{F}(s)^{*} \otimes \boldsymbol{\omega}(s)\right) \rightarrow \operatorname{Hom}\left(\mathcal{F}(s)^{*}, \mathcal{F}(s)^{*} \otimes \omega(s)\right)$

is zero. 
Since $Y$ is minimum, there is an injection $I_{Y}(s) \hookrightarrow \mathcal{F}^{*}(s) \otimes \boldsymbol{\omega}(s)$. Since the above bilinear map is zero, we must have $\operatorname{Hom}\left(\mathcal{F}(s)^{*}, I_{Y}(s)\right)=0$. By $(9.1)$,

$$
\chi(I(s) \otimes \mathcal{F}(s)) \geq \chi\left(I_{Y}(s) \otimes \mathcal{F}(s)\right),
$$

with equality if and only if $h^{0}(X(s), I(s) \otimes \mathcal{F}(s))=0$. Since by hypothesis we have the reverse inequality, it follows that $h^{0}(X(s), I(s) \otimes \mathcal{F}(s))=0$.

Let $X_{1}, \ldots, X_{n}$ be the irreducible components of $X$. By assumption, the $X_{i}$ are geometrically integral. For every $n$-uple $\underline{d}=\left(d_{1}, \ldots, d_{n}\right)$ of integers $d_{i}$, let $P^{\underline{d}} \subseteq P$ denote the open subscheme parameterizing invertible sheaves $L$ on $X$ whose restrictions to $X_{i}$ have degree $d_{i}$ for $i=1, \ldots, n$. The subschemes $P^{\underline{d}}$ are geometrically integral, and are the connected components of $P$.

Lemma 10. Fix an integer $m \geq 2$. For $i=1, \ldots, m$, let $\underline{d}^{i}$ be an $n$-uple of integers and $U_{i} \subseteq P^{\underline{d}^{i}}$ a dense, open subset. Let $\underline{d}:=\sum_{i} \underline{d}^{i}$ and $L$ an invertible sheaf on $X$ represented in $P \underline{d}$. Then, there are a finite and separable field extension $k^{\prime} \supseteq k$, and invertible sheaves $L_{i}$ on $X\left(k^{\prime}\right)$ represented by $k^{\prime}$-points of $U_{i}$ for $i=1, \ldots, m$, such that $L\left(k^{\prime}\right) \cong L_{1} \otimes \cdots \otimes L_{m}$.

Proof. Analogous to that of [9, Lemma 4, p. 185].

The following theorem gives a cohomological characterization of semi-stability.

Theorem 11. Let $k_{1} \supseteq k$ be a field extension and $I$ a torsion-free, rank-1 sheaf on $X\left(k_{1}\right)$ with $\chi(I)=d$. Fix an integer $m \geq 2$. Then, $I$ is semi-stable with respect to $E\left(k_{1}\right)$ if and only if there are a field extension $k^{\prime} \supseteq k$ and a vector bundle $F$ on $X\left(k^{\prime}\right)$ of rank $m r$ and $\operatorname{det} F \cong\left(\operatorname{det} E\left(k^{\prime}\right)\right)^{\otimes m}$ such that

$$
h^{0}\left(X\left(k_{2}\right), I\left(k_{2}\right) \otimes F\left(k_{2}\right)\right)=h^{1}\left(X\left(k_{2}\right), I\left(k_{2}\right) \otimes F\left(k_{2}\right)\right)=0
$$

for every field extension $k_{2} \supseteq k$ containing $k_{1}$ and $k^{\prime}$. In fact, we may take $k^{\prime} \supseteq k$ to be finite and separable.

Proof. We prove first the "only if" part. Let $i=1, m-1$. Applying Lemma 8 to $E^{\oplus i}$ we obtain a connected, smooth $k$-scheme $S_{i}$, a $k$-point $x_{i} \in S_{i}$ and an $S_{i^{-}}$ complete vector bundle $\mathcal{F}_{i}$ on $X \times S_{i}$ with $\mathcal{F}_{i}\left(x_{i}\right) \cong E^{\oplus i}$. Since $I$ is semi-stable with respect to $E$,

$$
\chi\left(I_{Y}(t) \otimes \mathcal{F}_{i}(t)\right)=\chi\left(I_{Y} \otimes E\left(k_{1}\right)^{\oplus i}\right) \geq 0=\chi\left(I \otimes E\left(k_{1}\right)^{\oplus i}\right)=\chi\left(I(t) \otimes \mathcal{F}_{i}(t)\right)
$$

for every subcurve $Y \subseteq X\left(k_{1}\right)$ and every $t \in S_{i}\left(k_{1}\right)$. By Lemma 9 , there is a dense, open subset $W_{i} \subseteq S_{i}\left(k_{1}\right)$ such that $h^{0}\left(X(t), I(t) \otimes \mathcal{F}_{i}(t)\right)=0$ for every $t \in W_{i}$. Since $\chi\left(I(t) \otimes \mathcal{F}_{i}(t)\right)=0$, we have $h^{1}\left(X(t), I(t) \otimes \mathcal{F}_{i}(t)\right)=0$ as well. Let $U_{i}$ denote the image of $W_{i}$ in $S_{i}$.

Let $\pi_{\mathcal{F}_{i}}: S_{i} \rightarrow P$ be the determinant map of $\mathcal{F}_{i}$ for $i=1, m-1$. The image $V_{i}:=\pi_{\mathcal{F}_{i}}\left(U_{i}\right)$ is open in $P$ because $\pi_{\mathcal{F}_{i}}$ is smooth. By Lemma 10 , there are a finite and separable field extension $k^{\prime} \supseteq k$, and invertible sheaves $L_{1}$ and $L_{m-1}$ on $X\left(k^{\prime}\right)$ represented in $P\left(k^{\prime}\right)$ by points $t_{1} \in V_{1}\left(k^{\prime}\right)$ and $t_{m-1} \in V_{m-1}\left(k^{\prime}\right)$, respectively, such that $L_{1} \otimes L_{m-1} \cong\left(\operatorname{det} E\left(k^{\prime}\right)\right)^{\otimes m}$. Since $\pi_{\mathcal{F}_{1}}$ and $\pi_{\mathcal{F}_{m-1}}$ are smooth, up to replacing $k^{\prime}$ by a finite and separable field extension, we may assume $t_{1}$ and $t_{m-1}$ lift to points $s_{1} \in U_{1}\left(k^{\prime}\right)$ and $s_{m-1} \in U_{m-1}\left(k^{\prime}\right)$, respectively. So,

$$
\left(\operatorname{det} \mathcal{F}_{1}\left(s_{1}\right)\right) \otimes\left(\operatorname{det} \mathcal{F}_{m-1}\left(s_{m-1}\right)\right) \cong\left(\operatorname{det} E\left(k^{\prime}\right)\right)^{\otimes m} .
$$

Let $F:=\mathcal{F}_{1}\left(s_{1}\right) \oplus \mathcal{F}_{m-1}\left(s_{m-1}\right)$. Then, $k^{\prime}$ and $F$ are as stated in the theorem. 
We prove now the "if" part. We may assume $k=k^{\prime}=k_{1}=k_{2}$. We use Nori's simple argument in the proof of [26, Lemma 8.3, p. 195]. Assume by contradiction that there is a vector bundle $F$ on $X$ of $\operatorname{rank} m r$ and $\operatorname{det} F \cong(\operatorname{det} E)^{\otimes m}$ such that

$$
h^{0}(X, I \otimes F)=h^{1}(X, I \otimes F)=0,
$$

but $I$ is not semi-stable. By definition, there is a non-empty, proper subcurve $Y \varsubsetneqq X$ such that $\chi\left(I_{Y} \otimes E\right)<0$. Letting $J:=\operatorname{ker}\left(I \rightarrow I_{Y}\right)$, we have $\chi(J \otimes E)>0$. Since the rank of $F$ is $m r$ and $\operatorname{det} F \cong(\operatorname{det} E)^{\otimes m}$, we have

$$
\chi(J \otimes F)=m \chi(J \otimes E)>0 .
$$

Thus, $h^{0}(X, J \otimes F)>0$. Since $J \otimes F \subseteq I \otimes F$ and $H^{0}(X, I \otimes F)=0$, we have a contradiction.

Let $I$ be a semi-stable sheaf on $X$ with respect to $E$ and $W \subseteq X$ an irreducible component. Let $Y \subseteq X$ be a subcurve containing $W$ such that $I_{Y}$ is semi-stable. If $Y$ is minimal with the above property, then $Y$ is minimum. In fact, if $Z \subseteq X$ is a subcurve with the same property, then so is $Y \wedge Z$ by Lemma 3. If $Y$ is minimal, then $Y \subseteq Z$.

Lemma 12. Let $p \in X$ be a smooth $k$-point and $Y \subseteq X$ a subcurve. Let $k_{1} \supseteq k$ be a field extension and $I$ a semi-stable sheaf on $X\left(k_{1}\right)$ with respect to $E\left(k_{1}\right)$. Fix an integer $m \geq 2$. Then, $Y$ is the minimum subcurve such that $p \in Y$ and $I_{Y\left(k_{1}\right)}$ is semi-stable if and only if there are a field extension $k^{\prime} \supseteq k$ and a vector bundle $F$ on $X\left(k^{\prime}\right)$ of rank $m r$ and $\operatorname{det} F \cong\left(\operatorname{det} E\left(k^{\prime}\right)\right)^{\otimes m} \otimes \mathcal{O}_{X}(-p)\left(k^{\prime}\right)$ such that, for every field extension $k_{2} \supseteq k$ containing $k_{1}$ and $k^{\prime}$,

(1) $h^{0}\left(X\left(k_{2}\right), I\left(k_{2}\right) \otimes F\left(k_{2}\right)\right)=h^{0}\left(X\left(k_{2}\right), I\left(k_{2}\right)_{Y\left(k_{2}\right)} \otimes F\left(k_{2}\right)\right)=0$,

(2) $h^{1}\left(X\left(k_{2}\right), I\left(k_{2}\right) \otimes F\left(k_{2}\right)\right)=1$, and the image of the unique (modulo $k_{2}^{*}$ ) nonzero map $I\left(k_{2}\right) \rightarrow F^{*}\left(k_{2}\right) \otimes \omega\left(k_{2}\right)$ is isomorphic to $I\left(k_{2}\right)_{Y\left(k_{2}\right)}$.

In fact, we may take $k^{\prime} \supseteq k$ to be finite and separable.

Proof. We prove first the "only if" part. By Lemma 8, there are a connected, smooth $k$-scheme $S$, an $S$-complete vector bundle $\mathcal{F}$ on $X \times S$ and a $k$-point $x \in S$ such that

$$
\mathcal{F}(x) \cong E^{\oplus m-2} \oplus \mathcal{O}_{X}^{\oplus r-1} \oplus\left((\operatorname{det} E) \otimes \mathcal{O}_{X}(-p)\right)
$$

If $Z \subseteq X$ is a subcurve and $t \in S\left(k_{1}\right)$, then

$$
\chi\left(I(t)_{Z(t)} \otimes \mathcal{F}(t)\right)=(m-1) \chi\left(I_{Z\left(k_{1}\right)} \otimes E\left(k_{1}\right)\right)-\delta(Z, p),
$$

where $\delta(Z, p)=1$ if $p \in Z$, and $\delta(Z, p)=0$ otherwise. Since $I$ is semi-stable, we have $\chi\left(I(t)_{Z(t)} \otimes \mathcal{F}(t)\right) \geq \chi(I(t) \otimes \mathcal{F}(t))$. By Lemma 9 , there are an open, dense subset $W \subseteq S\left(k_{1}\right)$ and a subcurve $Z \subseteq X$ such that, for every $t \in W$,

$$
h^{0}(X(t), I(t) \otimes \mathcal{F}(t))=h^{0}\left(X(t), I(t)_{Z(t)} \otimes \mathcal{F}(t)\right)=0,
$$

and $Z$ is the minimum subcurve of $X$ such that all maps $I(t) \rightarrow \mathcal{F}^{*}(t) \otimes \omega(t)$ factor through $I(t)_{Z(t)}$. Since $\chi(I(t) \otimes \mathcal{F}(t))=-1$ by (12.1), it follows from (12.2) that $h^{1}(X(t), I(t) \otimes \mathcal{F}(t))=1$. So, there is a unique (modulo $k(t)^{*}$ ) non-zero map $\lambda_{t}: I(t) \rightarrow \mathcal{F}^{*}(t) \otimes \omega(t)$, and $I(t)_{Z(t)} \cong \operatorname{im}\left(\lambda_{t}\right)$. Thus, by (12.1) and (12.2),

$$
(m-1) \chi\left(I_{Z\left(k_{1}\right)} \otimes E\left(k_{1}\right)\right)=\delta(Z, p)-1 .
$$


Since $I$ is semi-stable and $m>1$, we have $p \in Z$ and $I_{Z\left(k_{1}\right)}$ is semi-stable. On the other hand, $h^{1}\left(X(t), I(t)_{Y(t)} \otimes \mathcal{F}(t)\right) \geq 1$, because $\chi\left(I(t)_{Y(t)} \otimes \mathcal{F}(t)\right)=-1$ by (12.1). Since $I(t)_{Z(t)} \cong \operatorname{im}\left(\lambda_{t}\right)$, we have $Y \supseteq Z$. Since $Y$ is minimum, $Y=Z$.

Let $S_{1}$ be the connected, smooth $k$-scheme and $\mathcal{F}_{1}$ the $S_{1}$-complete vector bundle on $X \times S_{1}$ obtained in the proof of Theorem 11. We saw that there is a dense, open subset $W_{1} \subseteq S_{1}\left(k_{1}\right)$ such that

$$
h^{0}\left(X(t), I(t) \otimes \mathcal{F}_{1}(t)\right)=h^{1}\left(X(t), I(t) \otimes \mathcal{F}_{1}(t)\right)=0
$$

for every $t \in W_{1}$. Let $U$ and $U_{1}$ denote the respective images of $W$ and $W_{1}$ in $S$. As in the proof of Theorem 11, there are a finite and separable field extension $k^{\prime} \supseteq k$ and $k^{\prime}$-points $s \in U\left(k^{\prime}\right)$ and $s_{1} \in U_{1}\left(k^{\prime}\right)$ such that

$$
(\operatorname{det} \mathcal{F}(s)) \otimes\left(\operatorname{det} \mathcal{F}_{1}\left(s_{1}\right)\right) \cong\left(\operatorname{det} E\left(k^{\prime}\right)\right)^{\otimes m} \otimes \mathcal{O}_{X}(-p)\left(k^{\prime}\right) .
$$

Let $F:=\mathcal{F}(s) \oplus \mathcal{F}_{1}\left(s_{1}\right)$. Then, $k^{\prime}$ and $F$ are as stated in the theorem.

We prove now the "if" part. We may assume $k=k^{\prime}=k_{1}=k_{2}$. By (1) and (2), we have $\chi\left(I_{Y} \otimes F\right)=-1$. Since $F$ has rank $m r$ and $\operatorname{det} F \cong(\operatorname{det} E)^{\otimes m} \otimes \mathcal{O}_{X}(-p)$, we have

$$
\chi\left(I_{Z} \otimes F\right)=m \chi\left(I_{Z} \otimes E\right)-\delta(Z, p)
$$

for every subcurve $Z \subseteq X$. Since $I$ is semi-stable, $p \in Y$ and $I_{Y}$ is semi-stable. On the other hand, if $p \in Z$ and $I_{Z}$ is semi-stable, then $\chi\left(I_{Z} \otimes F\right)=-1$. So, $h^{1}\left(Z, I_{Z} \otimes F\right) \geq 1$. By (2), we have $Z \supseteq Y$. So, $Y$ is the minimum subcurve such that $p \in Y$ and $I_{Y}$ is semi-stable.

For every coherent sheaf $I$ on $X$, let

$$
e(I):=\max _{p \in X} \operatorname{dim}_{k(p)} I(p) .
$$

Proposition 13. Let $W \subseteq X$ be an irreducible component. If $I$ is a simple, torsion-free, rank-1 sheaf on $X$, then there are a finite and separable field extension $k^{\prime} \supseteq k$ and a vector bundle $E$ on $X\left(k^{\prime}\right)$ of rank $r:=\max \left(e\left(\underline{\operatorname{Hom}}_{X}(I, \omega)\right), 2\right)$ such that $I\left(k^{\prime}\right)$ is $W\left(k^{\prime}\right)$-quasi-stable with respect to $E$.

Proof. Applying the same method used in the proof of [9] Prop. 1], we get a finite and separable field extension $k^{\prime} \supseteq k$ and a vector bundle $F$ on $X\left(k^{\prime}\right)$ with rank $r$ and degree $-r \chi(I)-1$ such that

$$
h^{0}\left(X\left(k^{\prime}\right), I\left(k^{\prime}\right) \otimes F\right)=0 \quad \text { and } \quad h^{0}\left(X\left(k^{\prime}\right), I\left(k^{\prime}\right) \otimes F\right)=1,
$$

and the unique (modulo $k^{\prime *}$ ) non-zero map $\lambda: I\left(k^{\prime}\right) \rightarrow F^{*} \otimes \omega\left(k^{\prime}\right)$ is injective. (Note: here we use that $I$ is simple.) Up to replacing $k^{\prime}$ by a finite and separable field extension, we may assume there is a smooth $k^{\prime}$-point $p \in W\left(k^{\prime}\right)$. Let

$$
E:=\operatorname{ker}\left(F^{*} \rightarrow F^{*}(p) \stackrel{q}{\rightarrow} k^{\prime}\right)^{*},
$$

where $q$ is a $k^{\prime}$-linear surjection such that $q \circ \lambda(p) \neq 0$. (We chose implicitly a trivialization of $\omega\left(k^{\prime}\right)$ at $p$.) It is clear that $E$ has rank $r$ and degree $-r \chi(I)$. In fact, $\operatorname{det} E \cong(\operatorname{det} F) \otimes \mathcal{O}_{X\left(k^{\prime}\right)}(p)$. In addition, by our choice of $q$,

$$
h^{0}\left(X\left(k^{\prime}\right), I\left(k^{\prime}\right) \otimes E\right)=h^{1}\left(X\left(k^{\prime}\right), I\left(k^{\prime}\right) \otimes E\right)=0 .
$$

By Theorem 11, the sheaf $I\left(k^{\prime}\right)$ is semi-stable with respect to $E$. Moreover, $I\left(k^{\prime}\right)$ is $W\left(k^{\prime}\right)$-quasi-stable with respect to $E$ by Lemma 12 . 
Remark 14. Assume $k$ is algebraically closed. Given an irreducible component $W$ of $X$, it seems that a simple, torsion-free, rank- 1 sheaf $I$ on $X$ is $W$-quasi-stable with respect to a line bundle. At least, this can be shown when either

(1) $X$ is Gorenstein and $I$ is invertible, or

(2) $X$ has only ordinary nodes as singularities, or

(3) $X$ has at most two irreducible components.

Since this statement is not central to the present work, I will omit the proof of it in the above three cases.

Corollary 15. Let I be a simple, torsion-free, rank-1 sheaf on $X$. Let $n$ be the number of irreducible components of $X$ and $r:=\max \left(e\left(\underline{\operatorname{Hom}}_{X}(I, \omega)\right), 2\right)$. Then, there are a finite and separable field extension $k^{\prime} \supseteq k$ and a vector bundle $E$ on $X\left(k^{\prime}\right)$ of rank $n r$ such that $I\left(k^{\prime}\right)$ is stable with respect to $E$.

Proof. Let $X_{1}, \ldots, X_{n}$ denote the irreducible components of $X$. By Proposition 13 there are a finite and separable field extension $k^{\prime} \supseteq k$, and vector bundles $E_{i}$ on $X\left(k^{\prime}\right)$ of rank $r$ for $i=1, \ldots, n$, such that $I\left(k^{\prime}\right)$ is $X_{i}\left(k^{\prime}\right)$-quasi-stable with respect to $E_{i}$. Then, $I\left(k^{\prime}\right)$ is stable with respect to the direct sum $E:=E_{1} \oplus \cdots \oplus E_{n}$.

Remark 16. Assume $k$ is algebraically closed. If the expectation stated in Remark 14 is confirmed, then it follows that every simple, torsion-free, rank-1 sheaf on $X$ is stable with respect to a rank- $n$ vector bundle. At any rate, $n$ is a lower bound on the rank of the polarization: we can easily construct an example of a curve $X$ with $n$ irreducible components, and a simple, torsion-free, rank-1 sheaf $I$ on $X$ such that $I$ is not stable with respect to any vector bundle of rank less than $n$.

2.2. Families. Let $f: X \rightarrow S$ be a flat, projective map whose geometric fibers are curves. Let $\omega$ be a relative dualizing sheaf for $f$. Recall that $\omega$ is simple, torsion-free and rank-1 on $X / S$. Fix an integer $d$. Let $\mathcal{E}$ be a vector bundle on $X$ of rank $r$ and $\operatorname{deg}(\mathcal{E} / S)=-r d$.

Lemma 17. Let $\mathcal{L}$ be a line bundle on $X$ and $s \in S$. The following statements hold.

(1) If $F$ is a vector bundle on $X(s)$ with $\operatorname{det} F \cong \mathcal{L}(s)$, then there are an étale map $S^{\prime} \rightarrow S$, a vector bundle $\mathcal{F}$ on $X \times S^{\prime}$ and $s^{\prime} \in S^{\prime}$ lying over $s$ such that $\mathcal{F}\left(s^{\prime}\right) \cong F\left(s^{\prime}\right)$ and $\operatorname{det} \mathcal{F} \cong \mathcal{L} \otimes \mathcal{O}_{S^{\prime}}$.

(2) Let $\mathcal{O}_{X}(1)$ be an ample sheaf on $X / S$. Let $m$ be a positive integer, and $\mathcal{F}_{1}, \ldots, \mathcal{F}_{n}$ vector bundles on $X$ of rank $m$ and determinant $\mathcal{L}$. Then, there is an integer $c_{0}$ such that, for all $c \geq c_{0}$, there are an étale map $q_{c}: S_{c} \rightarrow S$ with $s \in q_{c}\left(S_{c}\right)$, and extensions

$$
0 \rightarrow p_{c}^{*} \mathcal{O}_{X}(-c)^{\oplus m-1} \rightarrow p_{c}^{*} \mathcal{F}_{i} \rightarrow p_{c}^{*} \mathcal{L}((m-1) c) \rightarrow 0
$$

for $i=1, \ldots, n$, where $p_{c}: X \times S_{c} \rightarrow X$ is the projection.

Proof. Let $\mathcal{O}_{X}(1)$ be an ample sheaf on $X / S$. We prove (1). Let $c$ be an integer such that $F(c)$ is generated by global sections. Let $m$ be the rank of $F$. Let $G$ be the Grassmannian of subspaces of dimension $m-1$ in $H^{0}(X(s), F(c))$. For each $p \in X(s)$ the subset $G_{p} \subseteq G$ parameterizing subspaces $V \subseteq H^{0}(X(s), F(c))$ such that the evaluation map $V \otimes k(p) \rightarrow F(c)(p)$ is not injective has codimension 2 . Since $X$ is a complete curve, the union $\bigcup_{p \in X} G_{p}$ is closed and of codimension at 
least $1 \mathrm{in} G$. So, there are a finite and separable field extension $k^{\prime} \supseteq k(s)$ and an exact sequence

$$
0 \rightarrow \mathcal{O}_{X}(-c)\left(k^{\prime}\right)^{\oplus m-1} \rightarrow F\left(k^{\prime}\right) \rightarrow N_{c} \rightarrow 0,
$$

where $N_{c}$ is an invertible sheaf on $X\left(k^{\prime}\right)$. (If $k(s)$ is infinite, we may take $k^{\prime}=k(s)$.) Replacing $S$ by an $S$-étale scheme, we may assume $k^{\prime}=k(s)$. Since $\operatorname{det} F \cong \mathcal{L}(s)$, we have $N_{c} \cong \mathcal{L}((m-1) c)(s)$.

Since the fibers of $f$ are curves, the formation of

$$
\operatorname{Ext}_{f}^{1}\left(\mathcal{L}((m-1) c), \mathcal{O}_{X}(-c)^{\oplus m-1}\right)
$$

commutes with base change. Thus, replacing $S$ by an open neighborhood of $s$, we may assume there is an extension

$$
0 \rightarrow \mathcal{O}_{X}(-c)^{\oplus m-1} \rightarrow \mathcal{F} \rightarrow \mathcal{L}((m-1) c) \rightarrow 0
$$

lifting (17.1). So $\mathcal{F}(s) \cong F$ and $\operatorname{det} \mathcal{F} \cong \mathcal{L}$.

We prove (2). We may assume $S$ is Noetherian. So, there is an integer $c_{0}$ such that, for $c \geq c_{0}$ and $i=1, \ldots, n$, the formation of $f_{*} \mathcal{F}_{i}(c)$ commutes with base change, and $\mathcal{F}_{i}(c)(s)$ is generated by global sections. Fix $c \geq c_{0}$. As before, up to replacing $S$ by an étale neighborhood of $s$, there are exact sequences

$$
0 \rightarrow \mathcal{O}_{X}(-c)(s)^{\oplus m-1} \rightarrow \mathcal{F}_{i}(s) \rightarrow N_{c, i} \rightarrow 0,
$$

where $N_{c, i}$ is invertible on $X(s)$ for $i=1, \ldots, n$. Since the formation of the $f_{*} \mathcal{F}_{i}(c)$ commutes with base change, up to replacing $S$ by a neighborhood of $s$, we may assume (17.2) is the restriction of an exact sequence

$$
0 \rightarrow \mathcal{O}_{X}(-c)^{\oplus m-1} \rightarrow \mathcal{F}_{i} \rightarrow \mathcal{N}_{c, i} \rightarrow 0
$$

for $i=1, \ldots, n$. Since $\operatorname{det} \mathcal{F}_{i} \cong \operatorname{det} \mathcal{L}$, we have $\mathcal{N}_{c, i} \cong \mathcal{L}((m-1) c)$ for every $i$.

Lemma 18. Let $s_{0} \in S$ be a closed point. There are an étale map $S^{\prime} \rightarrow S$ containing $s_{0}$ in its image, and $S$-maps $\sigma_{1}, \ldots, \sigma_{n}: S^{\prime} \rightarrow X$ such that

(1) $\sigma_{i}$ factors through the $S$-smooth locus of $X$;

(2) for every $s \in S^{\prime}$, every irreducible component of $X(s)$ is geometrically integral and contains $\sigma_{i}(s)$ for some $i$.

Proof. Replacing $S$ by an $S$-étale scheme, we may assume the irreducible components $X_{1}, \ldots, X_{n}$ of $X\left(s_{0}\right)$ are geometrically integral. For $i=1, \ldots, n$, let $Y_{i}:=X \backslash\left(\bigcup_{j \neq i} X_{j}\right)$. By [14, IV-4, 17.16.3], for each $i=1, \ldots, n$ there are an étale map $S_{i} \rightarrow S$ containing $s_{0}$ in its image, and an $S$-map $\sigma_{i}: S_{i} \rightarrow X$ factoring through the $S$-smooth locus of $Y_{i}$. Replacing $S$ by the fibered product of the $S_{i}$ over $S$, we may assume $S=S_{1}=\cdots=S_{n}$. By construction, $\sigma_{i}\left(s_{0}\right) \in X_{i}$ for $i=1, \ldots, n$. Thus, $\sigma_{1}\left(s_{0}\right)+\cdots+\sigma_{n}\left(s_{0}\right)$ is ample on $X\left(s_{0}\right)$. Since ampleness is an open property [14, III-1, 4.7.1, p. 145], replacing $S$ by an open neighborhood of $s$, we may assume $\sigma_{1}(s)+\cdots+\sigma_{n}(s)$ is ample on $X(s)$ for every $s \in S$. Consequently, for every $s \in S$ every irreducible component of $X(s)$ is geometrically integral and contains $\sigma_{i}(s)$ for some $i$.

Let $T$ be an $S$-scheme and $t \in T$. We say that maps $U \rightarrow S$ and $V \rightarrow T \times U$ form a neighborhood of $t$ in $T / S$ if the induced map $V \rightarrow T$ contains $t$ in its image. For short, we say that $V / U$ is a neighborhood of $t$ in $T / S$. The neighborhood $V / U$ is called étale if $U \rightarrow S$ and $V \rightarrow T \times U$ are étale. 
Theorems 19, 20, 21 and 22 are the (relative) cohomological characterizations of semi-stability, $\sigma$-quasi-stability, quasi-stability and stability, respectively.

Theorem 19. Let $T$ be an $S$-scheme and $\mathcal{I}$ a torsion-free, rank-1 sheaf on $X_{T} / T$ with $\chi(\mathcal{I} / T)=d$. Let $t \in T$. Fix an integer $m \geq 2$. Then, $\mathcal{I}(t)$ is semi-stable with respect to $\mathcal{E}(t)$ if and only if there are a neighborhood $V / U$ of $t$ in $T / S$ and a vector bundle $\mathcal{F}$ on $X_{U}$ of rank $m r$ and $\operatorname{det} \mathcal{F} \cong\left(\operatorname{det} \mathcal{E}_{U}\right)^{\otimes m}$ such that $R^{i} f_{V *}\left(\mathcal{I}_{V} \otimes \mathcal{F}_{V}\right)=0$ for every $i$. In fact, we may take the neighborhood $V / U$ of $t$ in $T / S$ to be étale.

Proof. Let $s \in S$ lying under $t$. By Lemma 18, up to replacing $S$ by an étale neighborhood of $s$ and $T$ by its product with this neighborhood, we may assume that the irreducible components of $X(s)$ are geometrically integral. Then the "if" statement is clear from Theorem 11.

Let's prove the "only if" statement. Since $\mathcal{I}(t)$ is semi-stable with respect to $\mathcal{E}(t)$, by Theorem 11, there are a finite and separable field extension $k \supseteq k(s)$ and a vector bundle $F$ on $X(k)$ of rank $m r$ and $\operatorname{det} F \cong(\operatorname{det} \mathcal{E}(k))^{\otimes m}$ such that

$$
h^{0}\left(X\left(k^{\prime}\right), \mathcal{I}\left(k^{\prime}\right) \otimes F\left(k^{\prime}\right)\right)=h^{1}\left(X\left(k^{\prime}\right), \mathcal{I}\left(k^{\prime}\right) \otimes F\left(k^{\prime}\right)\right)=0
$$

for every field extension $k^{\prime} \supseteq k(s)$ containing $k$ and $k(t)$. Up to replacing $S$ by an étale neighborhood of $s$ and $T$ by its product with this neighborhood, we may assume that $k=k(s)$. By Lemma 17, there are an étale map $U \rightarrow S$, a vector bundle $\mathcal{F}$ on $X_{U}$ and a point $u \in U$ lying over $s$ such that $\operatorname{det} \mathcal{F} \cong\left(\operatorname{det} \mathcal{E}_{U}\right)^{\otimes m}$ and $\mathcal{F}(u) \cong F(u)$. Let $v$ be a point of $T \times U$ lying over $(t, u)$ and $k^{\prime}:=k(v)$. Since (19.1) holds, by semicontinuity, there is an open neighborhood $V \subseteq T \times U$ of $v$ such that $R^{i} f_{V *}\left(\mathcal{I}_{V} \otimes \mathcal{F}_{V}\right)=0$ for every $i$.

Theorem 20. Let $\sigma: S \rightarrow X$ be a section through the smooth locus of $X / S$. Let $T$ be an $S$-scheme and $\mathcal{I}$ a semi-stable sheaf on $X_{T} / T$ with respect to $\mathcal{E}_{T}$. Let $t \in T$. Fix an integer $m \geq 2$. Then, $\mathcal{I}(t)$ is $\sigma(t)$-quasi-stable if and only if there are a neighborhood $V / U$ of $t$ in $T / S$ and a vector bundle $\mathcal{F}$ on $X_{U}$ of rank $m r$ and $\operatorname{det} \mathcal{F} \cong\left(\operatorname{det} \mathcal{E}_{U}\right)^{\otimes m} \otimes \mathcal{O}_{X_{U}}(-\sigma(S) \times U)$ such that

(1) $f_{V *}\left(\mathcal{I}_{V} \otimes \mathcal{F}_{V}\right)=0$ and $R^{1} f_{V *}\left(\mathcal{I}_{V} \otimes \mathcal{F}_{V}\right)$ is invertible,

(2) the natural map

$$
\mathcal{I}_{V} \longrightarrow \mathcal{F}_{V}^{*} \otimes \omega_{V} \otimes f_{V}^{*} R^{1} f_{V *}\left(\mathcal{I}_{V} \otimes \mathcal{F}_{V}\right)
$$

is injective with $V$-flat cokernel.

In fact, we may take the neighborhood $V / U$ of $t$ in $T / S$ to be étale.

Proof. The theorem is proved from Lemma 12 in the same way as Theorem 19 was proved from Theorem 11.

Theorem 21. Let $T$ be an $S$-scheme and $\mathcal{I}$ a semi-stable sheaf on $X_{T} / T$ with respect to $\mathcal{E}_{T}$. Let $t \in T$. Fix an integer $m \geq 2$. Then, $\mathcal{I}(t)$ is quasi-stable if and only if there are a neighborhood $V / U$ of $t$ in $T / S$, a section $\sigma: U \rightarrow X_{U}$ through the smooth locus of $X_{U} / U$ and a vector bundle $\mathcal{F}$ on $X_{U}$ of rank $m r$ and $\operatorname{det} \mathcal{F} \cong\left(\operatorname{det} \mathcal{E}_{U}\right)^{\otimes m} \otimes \mathcal{O}_{X_{U}}(-\sigma(U))$ such that

(1) $f_{V *}\left(\mathcal{I}_{V} \otimes \mathcal{F}_{V}\right)=0$ and $R^{1} f_{V *}\left(\mathcal{I}_{V} \otimes \mathcal{F}_{V}\right)$ is invertible,

(2) the natural map

$$
\mathcal{I}_{V} \longrightarrow \mathcal{F}_{V}^{*} \otimes \omega_{V} \otimes f_{V}^{*} R^{1} f_{V *}\left(\mathcal{I}_{V} \otimes \mathcal{F}_{V}\right)
$$

is injective with $V$-flat cokernel. 
In fact, we may take the neighborhood $V / U$ of $t$ in $T / S$ to be étale.

Proof. Let $s \in S$ lying under $t$. By Lemma 18, up to replacing $S$ by an étale neighborhood of $s$ and $T$ by its product with this neighborhood, we may assume that there are sections $\sigma_{1}, \ldots, \sigma_{n}: S \rightarrow X$ through the smooth locus of $X / S$ such that, for every $s^{\prime} \in S$, every irreducible component of $X\left(s^{\prime}\right)$ is geometrically integral and contains $\sigma_{i}\left(s^{\prime}\right)$ for some $i$. Now, $\mathcal{I}(t)$ is quasi-stable if and only if $\mathcal{I}(t)$ is $\sigma_{i}(t)$-quasi-stable for some $i$. We may now apply Theorem 20 .

Theorem 22. Let $T$ be an $S$-scheme and $\mathcal{I}$ a semi-stable sheaf on $X_{T} / T$ with respect to $\mathcal{E}_{T}$. Let $t \in T$. Fix an integer $m \geq 2$. Then, $\mathcal{I}(t)$ is stable if and only if for every map $S^{\prime} \rightarrow S$, every section $\sigma: S^{\prime} \rightarrow X_{S^{\prime}}$ through the smooth locus of $X_{S^{\prime}} / S^{\prime}$ and every point $t^{\prime} \in T \times S^{\prime}$ lying over $t$ there are a neighborhood $V / U$ of $t^{\prime}$ in $T \times S^{\prime} / S^{\prime}$ and a vector bundle $\mathcal{F}$ on $X_{U}$ of rank $m r$ and determinant $\left(\operatorname{det} \mathcal{E}_{U}\right)^{\otimes m} \otimes \mathcal{O}_{X_{U}}\left(-\sigma\left(S^{\prime}\right) \times U\right)$ such that

(1) $f_{V *}\left(\mathcal{I}_{V} \otimes \mathcal{F}_{V}\right)=0$ and $R^{1} f_{V *}\left(\mathcal{I}_{V} \otimes \mathcal{F}_{V}\right)$ is invertible,

(2) the natural map

$$
\mathcal{I}_{V} \longrightarrow \mathcal{F}_{V}^{*} \otimes \omega_{V} \otimes f_{V}^{*} R^{1} f_{V *}\left(\mathcal{I}_{V} \otimes \mathcal{F}_{V}\right)
$$

is injective with $V$-flat cokernel.

In fact, we may take the neighborhood $V / U$ of $t^{\prime}$ in $T \times S^{\prime} / S^{\prime}$ to be étale.

Proof. As in the proof of Theorem 21, we may assume that there are sections $\sigma_{1}, \ldots, \sigma_{n}: S \rightarrow X$ through the smooth locus of $X / S$ such that, for every $s \in S$, every irreducible component of $X(s)$ is geometrically integral and contains $\sigma_{i}(s)$ for some $i$. Now, $\mathcal{I}(t)$ is stable if and only if $\mathcal{I}(t)$ is $\sigma_{i, T}(t)$-quasi-stable for every $i$. We may now apply Theorem 20.

\section{The Valuative CRITERIA}

Throughout Section 3 we denote by $S$ the spectrum of a discrete valuation ring $R$. Let $\pi$ be a generator of the maximal ideal of $R$. Let $s$ be the special point of $S$, and $\eta$ its generic point. Let $X / S$ be a family of curves. Assume throughout Section 3 that the irreducible components of the special fiber $X(s)$ are geometrically integral.

If $\mathcal{I}$ is a torsion-free, rank-1 sheaf on $X / S$, and $Y \subseteq X(s)$ is a subcurve, then we denote by $\mathcal{I}^{Y}$ the kernel of the canonical surjection $\mathcal{I} \rightarrow \mathcal{I}(s)_{Y}$. It is clear that the inclusion $\lambda: \mathcal{I}^{Y} \hookrightarrow \mathcal{I}$ is an isomorphism on $X \backslash Y$. In addition, it can be shown, by using an argument analogous to the one found in [21, Prop. 6, p. 100], that $\mathcal{I}^{Y}$ is torsion-free, rank-1 on $X / S$.

Lemma 23. Let $\mathcal{I}$ be a torsion-free, rank-1 sheaf on $X / S$. Let $Y \subseteq X(s)$ be a subcurve and $Z:=Y^{c}$. The following statements hold.

(1) $\left(\mathcal{I}^{Y}\right)^{Z}=\mathcal{I}^{Y} \cap \mathcal{I}^{Z}=\pi \mathcal{I}$.

(2) There are natural short exact sequences

$$
\begin{aligned}
& 0 \rightarrow \mathcal{I}^{Y}(s)_{Z} \rightarrow \mathcal{I}(s) \rightarrow \mathcal{I}(s)_{Y} \rightarrow 0 \\
& 0 \rightarrow \mathcal{I}(s)_{Y} \rightarrow \mathcal{I}^{Y}(s) \rightarrow \mathcal{I}^{Y}(s)_{Z} \rightarrow 0
\end{aligned}
$$


Proof. Since $\mathcal{I} / \mathcal{I}^{Y}$ is supported on $Y$, the natural map $\mathcal{I}^{Y}(s)_{Z} \rightarrow \mathcal{I}(s)_{Z}$ is injective. Thus $\left(\mathcal{I}^{Y}\right)^{Z}=\mathcal{I}^{Y} \cap \mathcal{I}^{Z}$. In addition, since the natural map $\mathcal{I}(s) \rightarrow \mathcal{I}(s)_{Y} \oplus \mathcal{I}(s)_{Z}$ is injective, $\mathcal{I}^{Y} \cap \mathcal{I}^{Z}=\pi \mathcal{I}$.

We prove (2). Since the natural map $\mathcal{I}(s) \rightarrow \mathcal{I}(s)_{Y} \oplus \mathcal{I}(s)_{Z}$ is an injection, the kernel $H$ of the map $\mathcal{I}(s) \rightarrow \mathcal{I}(s)_{Y}$ is contained in $\mathcal{I}(s)_{Z}$. Hence $H$ is torsionfree and rank-1 on $Z$. From the definition of $\mathcal{I}^{Y}$ we get that $H$ is the image of $\mathcal{I}^{Y}(s) \rightarrow \mathcal{I}(s)$. Hence $\mathcal{I}^{Y}(s)_{Z} \cong H$, showing the exactness of (23.1). Since $\mathcal{I} \cong\left(\mathcal{I}^{Y}\right)^{Z}$ by (1), the exactness of (23.2) is shown in exactly the same way.

The following existence lemma is the main technical tool of this section. It will be used in the proof of Theorem 32 .

Lemma 24. Let $Y \subseteq X(s)$ be a subcurve. Let

$$
\cdots \subseteq \mathcal{I}^{i} \subseteq \cdots \subseteq \mathcal{I}^{1} \subseteq \mathcal{I}^{0}:=\mathcal{I}
$$

be an infinite filtration of $\mathcal{I}$ with quotients

$$
\frac{\mathcal{I}^{i}}{\mathcal{I}^{i+1}}=\mathcal{I}^{i}(s)_{Y}
$$

for every $i \geq 0$. If $R$ is complete, and $\mathcal{I}^{i}(s)$ decomposes at $Y$ for every $i \geq 0$, then there is an $S$-flat quotient $\mathcal{F}$ of $\mathcal{I}$ on $X$ such that $\mathcal{F}(s)=\mathcal{I}(s)_{Y}$.

Proof. The proof is analogous to the proof of [21, Lemma 2, p. 106]. For completeness, we give it below. For every $i \geq 0$, let $S_{i}:=\operatorname{Spec}\left(R / \pi^{i+1}\right)$ and $X_{i}:=X \times{ }_{S} S_{i}$. For every coherent sheaf $\mathcal{H}$ on $X$, let $H_{i}:=\left.\mathcal{H}\right|_{X_{i}}$ for every $i \geq 0$. Let $Z:=Y^{c}$.

We claim first that $\operatorname{im}\left(I_{l}^{i} \rightarrow I_{l}^{k}\right)=\operatorname{im}\left(I_{l}^{j} \rightarrow I_{l}^{k}\right)$ if $i \geq j>k \geq 0$ and $l \geq 0$, as long as $j-k>l$. Indeed, we argue by induction on $l$. Since $\mathcal{I}^{i}(s)$ decomposes at $Y$ for every $i \geq 0$, the inclusion $\mathcal{I}^{i+1} \hookrightarrow \mathcal{I}^{i}$ induces an isomorphism $I^{i+1}(s)_{Z} \rightarrow I^{i}(s)_{Z}$ for every $i \geq 0$. Thus, our claim holds for $l=0$. Assume now our claim holds for $l$. Let $i, j, k$ be integers with $i \geq j>k \geq 0$ and $j-k>l+1$. For every $m \geq 0$, there is a natural and functorial isomorphism $H_{0} \cong \pi^{m} H_{m}$ for every coherent sheaf $\mathcal{H}$ on $X$. So, we get a natural commutative diagram

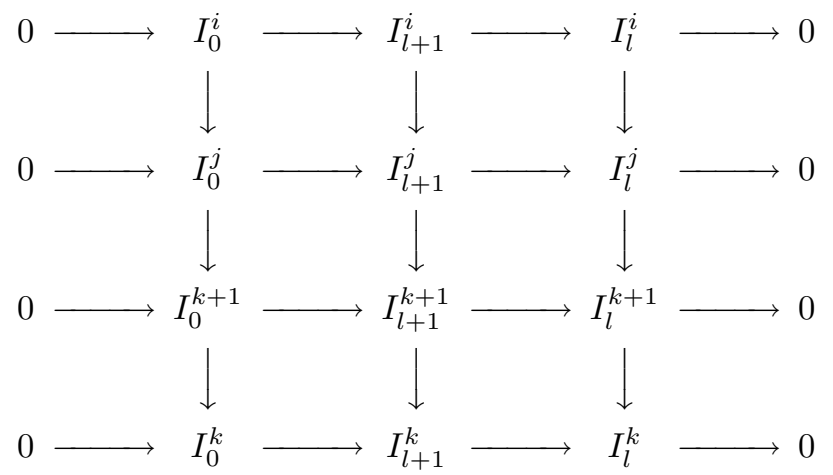

with exact rows. By induction hypothesis,

$$
\operatorname{im}\left(I_{l}^{i} \rightarrow I_{l}^{k+1}\right)=\operatorname{im}\left(I_{l}^{j} \rightarrow I_{l}^{k+1}\right) \quad \text { and } \quad \operatorname{im}\left(I_{0}^{i} \rightarrow I_{0}^{k}\right)=\operatorname{im}\left(I_{0}^{k+1} \rightarrow I_{0}^{k}\right) .
$$

Chasing diagram (24.1), we get that $\operatorname{im}\left(I_{l+1}^{i} \rightarrow I_{l+1}^{k}\right)=\operatorname{im}\left(I_{l+1}^{j} \rightarrow I_{l+1}^{k}\right)$. The proof of our first claim is complete. 
For every $i \geq 0$, let $F_{i}:=\operatorname{coker}\left(I_{i}^{i+1} \rightarrow I_{i}\right)$. We claim that $\left.F_{i}\right|_{X_{j}}=F_{j}$ if $i \geq j \geq 0$. Indeed, consider the natural commutative diagram

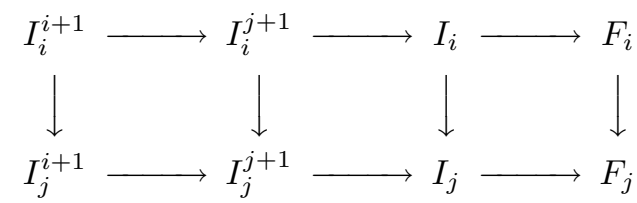

Of course, $F_{i} \rightarrow F_{j}$ is surjective. By our first claim, the images of $I_{j}^{i+1}$ and $I_{j}^{j+1}$ in $I_{j}$ are equal. Chasing diagram (24.2), we get that $\operatorname{ker}\left(I_{i} \rightarrow I_{j}\right) \rightarrow \operatorname{ker}\left(F_{i} \rightarrow F_{j}\right)$ is surjective. It follows that $\operatorname{ker}\left(F_{i} \rightarrow F_{j}\right)=\pi^{j+1} F_{i}$, proving our second claim.

For integers $i, j$ with $i, j \geq 0$, let $\mu_{i}^{j}: F_{i} \rightarrow F_{i}$ denote the multiplicationby- $\pi^{j}$ map. We claim now that $F_{i}$ is $S_{i}$-flat for every $i \geq 0$. Indeed, we argue by induction on $i$. If $i=0$, there is nothing to prove. Let $i, j$ be integers with $i \geq j \geq 1$. We need to show that $\operatorname{ker}\left(\mu_{i}^{j}\right)=\pi^{i+1-j} F_{i}$. Assume $F_{i-1}$ is $S_{i-1}$-flat. So, $\operatorname{ker}\left(\mu_{i-1}^{j}\right)=\pi^{i-j} F_{i-1}$. It follows that $\operatorname{ker}\left(\mu_{i}^{j}\right) \subseteq \pi^{i-j} F_{i}$. Thus, we need only show that $\operatorname{ker}\left(\mu_{i}^{i}\right)=\pi F_{i}$ for every $i \geq 0$.

Let $U \subseteq X$ be an affine open subset and $\tau \in \mathcal{I}(U)$. For every $i \geq 0$ let $\tau_{i} \in I_{i}(U)$ denote the restriction of $\tau$, and $\bar{\tau}_{i}$ be the class of $\tau_{i}$ in $F_{i}(U)$. Assume $\bar{\tau}_{i} \in \operatorname{ker}\left(\mu_{i}^{i}\right)$. So, there is $\mu \in \mathcal{I}^{i+1}(U)$ such that $\pi^{i} \tau-\mu \in \pi^{i+1} \mathcal{I}(U)$. Since $\pi^{i+1} \mathcal{I} \subseteq \mathcal{I}^{i+1}$, we have $\pi^{i} \tau \in \mathcal{I}^{i+1}(U)$. From (1) of Lemma 23 we get that

$$
\pi \mathcal{I}^{j} \cap \mathcal{I}^{j+2}=\pi \mathcal{I}^{j+1}
$$

for every $j \geq 0$. Since $\pi$ is a non-zero-divisor in $\mathcal{I}^{j}$ for every $j \geq 0$, applying (24.3) repeatedly, we get that $\pi^{i} \mathcal{I} \cap \mathcal{I}^{i+1}=\pi^{i} \mathcal{I}^{1}$. So $\tau \in \mathcal{I}^{1}(U)$. Since $\tau_{0} \in \operatorname{im}\left(I_{0}^{1} \rightarrow I_{0}\right)$, it follows from our first claim that $\tau_{0} \in \operatorname{im}\left(I_{0}^{i+1} \rightarrow I_{0}\right)$ as well. Thus there is $\gamma \in \mathcal{I}^{i+1}(U)$ such that $\tau-\gamma \in \pi \mathcal{I}$. So, $\bar{\tau}_{i} \in \pi F_{i}$, finishing the proof of our third claim.

By Grothendieck's existence theorem [14, III-1, 5.1.7], since $R$ is complete, there is a quotient $\mathcal{F}$ of $\mathcal{I}$ on $X$ such that $\mathcal{F}$ is the inverse limit of the $F_{i}$. Since each $F_{i}$ is $S_{i}$-flat, $\mathcal{F}$ is $S$-flat. Moreover, $\mathcal{F}(s)=F_{0}=\mathcal{I}(s)_{Y}$.

For the remainder of this section, fix an integer $d$ and a vector bundle $\mathcal{E}$ on $X$ of rank $r>0$ and relative degree $-r d$ over $S$. We consider $\mathcal{E}$ our relative polarization (see Subsection 1.4).

Lemma 25. Let $\mathcal{I}$ be a semi-stable sheaf on $X / S$. If $Y \subseteq X(s)$ is a subcurve, then $\mathcal{I}^{Y}$ is semi-stable on $X / S$ if and only if $\mathcal{I}(s)_{Y}$ is semi-stable with respect to $\left.\mathcal{E}(s)\right|_{Y}$. In this case, $\mathcal{I}^{Y}(s)$ is JH-equivalent to $\mathcal{I}(s)$.

Proof. Of course, $\mathcal{I}^{Y}$ is semi-stable over $S$ if and only if $\mathcal{I}^{Y}(s)$ is semi-stable. Let $Z:=Y^{c}$. If $\mathcal{I}^{Y}(s)$ is semi-stable, then so is $\mathcal{I}(s)_{Y}$, as the latter is the cokernel of the map $\mathcal{I}^{Y}(s) \rightarrow \mathcal{I}(s)$ of semi-stable sheaves by (23.1). On the other hand, if $\mathcal{I}(s)_{Y}$ is semi-stable, then so is $\mathcal{I}^{Y}(s)_{Z}$, again by (23.1). Since $\mathcal{I}^{Y}(s)$ is an extension of semi-stable sheaves by $(23.2)$, we conclude that $\mathcal{I}^{Y}(s)$ is semi-stable. In this case,

$$
\operatorname{Gr}\left(\mathcal{I}^{Y}(s)\right) \cong \operatorname{Gr}\left(\mathcal{I}(s)_{Y}\right) \oplus \operatorname{Gr}\left(\mathcal{I}^{Y}(s)_{Z}\right) \cong \operatorname{Gr}(\mathcal{I}(s)) .
$$

The following propositions will be used in Section 4 to show certain moduli spaces are separated over $S$ (see Theorem A). 
Proposition 26. Let $\mathcal{I}, \mathcal{J}$ be semi-stable sheaves on $X / S$ such that $\mathcal{I}(\eta) \cong \mathcal{J}(\eta)$. Then, $\mathcal{I}(s)$ and $\mathcal{J}(s)$ are JH-equivalent. In particular, $\mathcal{I}(s) \cong \mathcal{J}(s)$ if $\mathcal{I}(s)$ is stable.

Proof. As in the proof of [3 Lemma 7.8, p. 100], there is a map $\lambda: \mathcal{I} \rightarrow \mathcal{J}$ such that $\lambda(\eta)$ is an isomorphism, and $\lambda(s)$ is non-zero. Let $Y \subseteq X(s)$ be the subcurve such that $\mathcal{I}(s)_{Y} \cong \operatorname{im}(\lambda(s))$. Since $\lambda(s)$ is non-zero, $Y$ is non-empty. If $Y=X(s)$, then $\lambda(s)$ is an embedding. In this case, since $\lambda(\eta)$ is an isomorphism, so is $\lambda(s)$. We may thus assume $Y$ is a proper subcurve of $X$. Since $\mathcal{I}(s)$ and $\mathcal{J}(s)$ are semi-stable, so is $\mathcal{I}(s)_{Y}$. Since $\mathcal{I}(s)_{Y}$ and $\mathcal{J}(s)$ are semi-stable, so is $\mathcal{J}(s)_{Z}$, where $Z:=Y^{c}$. Let $\mathcal{J}_{1}:=\operatorname{ker}\left(\mathcal{J} \rightarrow \mathcal{J}(s)_{Z}\right)$. As $\mathcal{J}(s)_{Z}$ is semi-stable, by Lemma $25, \mathcal{J}_{1}$ is semi-stable on $X / S$, and $\mathcal{J}_{1}(s)$ is JH-equivalent to $\mathcal{J}(s)$. Moreover, $\lambda$ factors through $\mathcal{J}_{1}$ by construction. Applying the same procedure described above to the induced map $\lambda_{1}: \mathcal{I} \rightarrow \mathcal{J}_{1}$ in place of $\lambda$, and so on, we will eventually find a semi-stable subsheaf $\mathcal{J}_{m} \subseteq \mathcal{J}$ such that $\mathcal{J}_{m}(s)$ is JH-equivalent to $\mathcal{J}(s)$, and the induced map $\lambda_{m}: \mathcal{I} \rightarrow \mathcal{J}_{m}$ is an isomorphism.

Proposition 27. Let $\sigma: S \rightarrow X$ be a section through the smooth locus of $X / S$. Let $\mathcal{I}$ and $\mathcal{J}$ be $\sigma$-quasi-stable sheaves on $X / S$ with respect to $\mathcal{E}$. If $\mathcal{I}(\eta) \cong \mathcal{J}(\eta)$, then $\mathcal{I} \cong \mathcal{J}$.

Proof. As in the proof of [3, Lemma 7.8, p. 100], there are maps $\lambda: \mathcal{I} \rightarrow \mathcal{J}$ and $\mu: \mathcal{J} \rightarrow \mathcal{I}$ such that $\lambda(\eta)$ and $\mu(\eta)$ are isomorphisms, and $\lambda(s)$ and $\mu(s)$ are non-zero. Let $Y, Z \subseteq X(s)$ be subcurves such that $\mathcal{I}(s)_{Y} \cong \operatorname{im}(\lambda(s))$ and $\mathcal{J}(s)_{Z} \cong \operatorname{im}(\mu(s))$. Since $\lambda(s)$ and $\mu(s)$ are non-zero, $Y$ and $Z$ are non-empty. Since $\mathcal{I}(s)$ and $\mathcal{J}(s)$ are semi-stable, so are $\mathcal{I}(s)_{Z^{c}}$ and $\mathcal{J}(s)_{Y^{c}}$. Since $\mathcal{I}(s)$ and $\mathcal{J}(s)$ are $\sigma(s)$-quasi-stable, $\sigma(s) \notin Y^{c} \cup Z^{c}$. So, $\sigma(s) \in Y \wedge Z$. Since $Y \wedge Z \neq \emptyset$, the composition $\mu(s) \circ \lambda(s)$ is not zero. Since $\mathcal{I}(s)$ is simple, $\mu(s) \circ \lambda(s)$ is a homothety. Thus, $\lambda(s)$ is injective and, since $\lambda(\eta)$ is an isomorphism, $\lambda$ is an isomorphism.

The following four lemmas will be used in the proof of Theorem 32 .

Lemma 28. Let $\mathcal{I}$ be a torsion-free, rank-1 sheaf on $X / S$. Let $Y \subseteq X(s)$ be a subcurve such that $\mathcal{I}(s)$ decomposes at $Y$. Then, for any subcurve $Z \subseteq X$, if $\mathcal{I}^{Y}(s)$ decomposes at $Z$, so does $\mathcal{I}(s)$.

Proof. Restricting (23.2) to $Z$, and removing torsion, we get a natural, commutative diagram

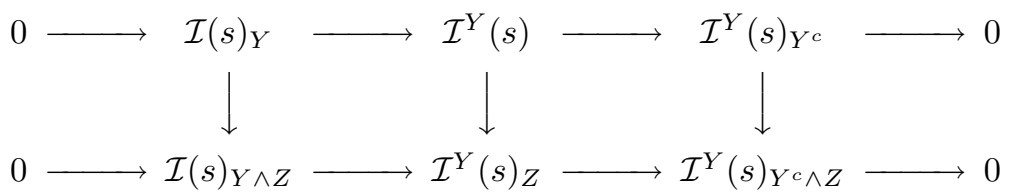

where the second row is exact but possibly at the middle. Combining the above diagram with that of $Z^{c}$, we get a natural, commutative diagram

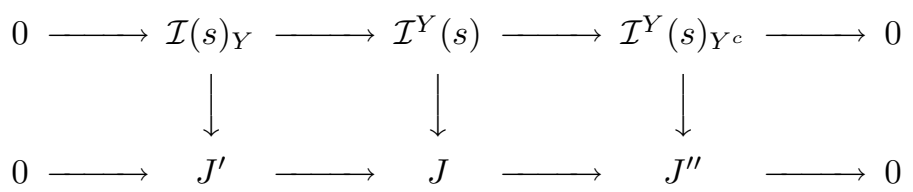

where $J:=\mathcal{I}^{Y}(s)_{Z} \oplus \mathcal{I}^{Y}(s)_{Z^{c}}$,

$$
J^{\prime}:=\mathcal{I}(s)_{Y \wedge Z} \oplus \mathcal{I}(s)_{Y \wedge Z^{c}} \quad \text { and } \quad J^{\prime \prime}:=\mathcal{I}^{Y}(s)_{Y^{c} \wedge Z} \oplus \mathcal{I}^{Y}(s)_{Y^{c} \wedge Z^{c}} .
$$


Assume $\mathcal{I}^{Y}(s)$ decomposes at $Z$. It follows from the snake lemma applied to (28.1) that $\mathcal{I}(s)_{Y}$ decomposes at $Y \wedge Z$ and $\mathcal{I}^{Y}(s)_{Y^{c}}$ decomposes at $Y^{c} \wedge Z$.

Since $\mathcal{I}(s)$ decomposes at $Y$, sequence (23.1) splits. So, the natural, commutative diagram

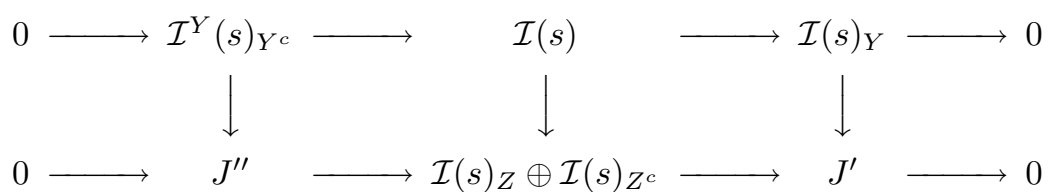

has exact rows. Since $\mathcal{I}(s)_{Y}=J^{\prime}$ and $\mathcal{I}^{Y}(s)_{Y^{c}}=J^{\prime \prime}$, it follows that $\mathcal{I}(s)$ decomposes at $Z$.

Let $I$ be a torsion-free, rank-1 sheaf on $X(s)$. If $Y, Z \subseteq X(s)$ are subcurves with $Y \wedge Z=\emptyset$, let

$$
\delta_{I}(Y, Z):=\chi\left(I_{Y}\right)+\chi\left(I_{Z}\right)-\chi\left(I_{Y \cup Z}\right) .
$$

If $Z^{\prime} \subseteq Z$ is a subcurve, then $\delta_{I}\left(Y, Z^{\prime}\right) \leq \delta_{I}(Y, Z)$ by Lemma 3. In particular, $\delta_{I}(Y, Z) \geq 0$, with equality if and only if $I_{Y \cup Z}=I_{Y} \oplus I_{Z}$.

Lemma 29. Let $\mathcal{I}$ be a torsion-free, rank-1 sheaf on $X / S$ with $\chi(\mathcal{I} / S)=d$. Let $Y, Z \subseteq X$ be subcurves. Then,

$$
\beta_{\mathcal{I}^{Y}(s)}(Z)+\beta_{\mathcal{I}(s)}(Y) \geq \beta_{\mathcal{I}(s)}(Y \wedge Z)+\beta_{\mathcal{I}(s)}(Y \cup Z)
$$

with equality if and only if $\delta_{\mathcal{I}^{Y}(s)}\left(Y \wedge Z, Y^{c}\right)=\delta_{\mathcal{I}^{Y}(s)}\left(Y \wedge Z, Y^{c} \wedge Z\right)$.

Proof. Restricting (23.1) and (23.2) to $Y \cup Z$ and $Y^{c} \cup Z$ respectively, and removing torsion, we get exact sequences

$$
\begin{aligned}
& 0 \rightarrow \mathcal{I}^{Y}(s)_{Y^{c} \wedge Z} \rightarrow \mathcal{I}(s)_{Y \cup Z} \rightarrow \mathcal{I}(s)_{Y} \rightarrow 0, \\
& 0 \rightarrow \mathcal{I}(s)_{Y \wedge Z} \rightarrow \mathcal{I}^{Y}(s)_{Y^{c} \cup Z} \rightarrow \mathcal{I}^{Y}(s)_{Y^{c}} \rightarrow 0 .
\end{aligned}
$$

It follows from the above exact sequences that

$$
\begin{aligned}
\beta_{\mathcal{I}^{Y}(s)}\left(Y^{c} \wedge Z\right) & =\beta_{\mathcal{I}(s)}\left(Y^{c} \wedge Z\right)-\delta_{\mathcal{I}(s)}\left(Y^{c} \wedge Z, Y\right), \\
\beta_{\mathcal{I}^{Y}(s)}(Y \wedge Z) & =\beta_{\mathcal{I}(s)}(Y \wedge Z)+\delta_{\mathcal{I}^{Y}(s)}\left(Y \wedge Z, Y^{c}\right) .
\end{aligned}
$$

Hence,

$$
\begin{aligned}
\beta_{\mathcal{I}^{Y}(s)}(Z) & =\beta_{\mathcal{I}^{Y}(s)}(Y \wedge Z)+\beta_{\mathcal{I}^{Y}(s)}\left(Y^{c} \wedge Z\right)-\delta_{\mathcal{I}^{Y}(s)}\left(Y \wedge Z, Y^{c} \wedge Z\right) \\
& =\beta_{\mathcal{I}(s)}(Y \wedge Z)+\beta_{\mathcal{I}(s)}\left(Y^{c} \wedge Z\right)-\delta_{\mathcal{I}^{Y}(s)}\left(Y \wedge Z, Y^{c} \wedge Z\right) \\
& +\delta_{\mathcal{I}^{Y}(s)}\left(Y \wedge Z, Y^{c}\right)-\delta_{\mathcal{I}^{(s)}}\left(Y^{c} \wedge Z, Y\right) \\
& \geq \beta_{\mathcal{I}(s)}(Y \wedge Z)+\beta_{\mathcal{I}(s)}\left(Y^{c} \wedge Z\right)-\delta_{\mathcal{I}(s)}\left(Y^{c} \wedge Z, Y\right) \\
& =\beta_{\mathcal{I}(s)}(Y \wedge Z)-\beta_{\mathcal{I}(s)}(Y)+\beta_{\mathcal{I}(s)}(Y \cup Z),
\end{aligned}
$$

with equality if and only if $\delta_{\mathcal{I}^{Y}(s)}\left(Y \wedge Z, Y^{c}\right)=\delta_{\mathcal{I}^{Y}(s)}\left(Y \wedge Z, Y^{c} \wedge Z\right)$.

Let $I$ be a torsion-free, rank-1 sheaf on $X(s)$ with $\chi(I)=d$. It follows easily from Lemma 3 that there is a maximum subcurve $Y \subseteq X(s)$ among the subcurves $W \subseteq X(s)$ with minimum $\beta_{I}(W)$. It is clear that $I$ is semi-stable if and only if $Y=X(s)$. 
Lemma 30. Let $\mathcal{I}$ be a torsion-free, rank-1 sheaf on $X / S$ with $\chi(\mathcal{I} / S)=d$. Let $Y \subseteq X(s)$ be the maximum subcurve among the subcurves $W \subseteq X(s)$ with minimum $\beta_{\mathcal{I}(s)}(W)$. Then, $\beta_{\mathcal{I}^{Y}(s)}(Z) \geq \beta_{\mathcal{I}(s)}(Y)$ for every subcurve $Z \subseteq X(s)$, with equality only if $Z \subseteq Y$. Moreover, $\beta_{\mathcal{I}^{Y}(s)}(Y)=\beta_{\mathcal{I}(s)}(Y)$ if and only if $\mathcal{I}^{Y}(s)$ decomposes at $Y$.

Proof. Let $Z \subseteq X(s)$ be a subcurve. Since $\beta_{\mathcal{I}(s)}(Y)$ is minimum, by Lemma 29,

$$
\beta_{\mathcal{I}^{Y}(s)}(Z) \geq \beta_{\mathcal{I}(s)}(Y \wedge Z)-\beta_{\mathcal{I}(s)}(Y)+\beta_{\mathcal{I}(s)}(Y \cup Z) \geq \beta_{\mathcal{I}(s)}(Y),
$$

with equality if and only if

$$
\begin{aligned}
\delta_{\mathcal{I}^{Y}(s)}\left(Y \wedge Z, Y^{c}\right) & =\delta_{\mathcal{I}^{Y}(s)}\left(Y \wedge Z, Y^{c} \wedge Z\right), \\
\beta_{\mathcal{I}(s)}(Y \cup Z) & =\beta_{\mathcal{I}(s)}(Y), \\
\beta_{\mathcal{I}(s)}(Y \wedge Z) & =\beta_{\mathcal{I}(s)}(Y) .
\end{aligned}
$$

Since $Y$ is maximum among the subcurves $W \subseteq X(s)$ with minimum $\beta_{\mathcal{I}(s)}(W)$, the middle equality above occurs if and only if $Z \subseteq Y$. The first statement of the lemma is proved. If $Z=Y$, then the last two equalities above are obviously satisfied, whereas the first equality is satisfied if and only if $\delta_{\mathcal{I}^{Y}(s)}\left(Y, Y^{c}\right)=0$.

Lemma 31. Let $\mathcal{I}$ be a semi-stable sheaf on $X / S$ with respect to $\mathcal{E}$. Fix an irreducible component $W \subseteq X(s)$, and let $Y \subseteq X(s)$ be the minimum subcurve containing $W$ such that $\beta_{\mathcal{I}(s)}(Y)=0$. Then, $\mathcal{I}^{\bar{Y}}$ is also semi-stable on $X / S$ with respect to $\mathcal{E}$. Moreover, if $Z \subseteq X(s)$ is the minimum subcurve containing $W$ such that $\beta_{\mathcal{I}^{Y}(s)}(Z)=0$, then $Z \supseteq Y$, with equality $Z=Y$ if and only if $\mathcal{I}^{Y}(s)$ decomposes at $Y$.

Proof. The first statement follows directly from Lemma 25. As for the second one, since $\mathcal{I}(s)$ is semi-stable, $\beta_{\mathcal{I}(s)}(Y)=0$ and $\beta_{\mathcal{I}^{Y}(s)}(Z)=0$, by Lemma 29 ,

$$
0=\beta_{\mathcal{I}^{Y}(s)}(Z) \geq \beta_{\mathcal{I}(s)}(Y \wedge Z)+\beta_{\mathcal{I}(s)}(Y \cup Z) \geq 0 .
$$

So, $\beta_{\mathcal{I}(s)}(Y \wedge Z)=0$ and, by Lemma 29 again,

$$
\delta_{\mathcal{I}^{Y}(s)}\left(Y \wedge Z, Y^{c}\right)=\delta_{\mathcal{I}^{Y}(s)}\left(Y \wedge Z, Y^{c} \wedge Z\right) .
$$

Since $Z \supseteq W$, and $Y$ is the minimum subcurve containing $W$ with $\beta_{\mathcal{I}(s)}(Y)=0$, it follows that $Z \supseteq Y$. The rest of the second statement follows now from (31.1).

Let $I_{\eta}$ be a torsion-free, rank-1 sheaf on $X(\eta)$. We say that a torsion-free, rank-1 sheaf $\mathcal{I}$ on $X / S$ is an extension of $I_{\eta}$ if $\mathcal{I}(\eta) \cong I_{\eta}$.

The following theorem will be used in Section 4 to show certain moduli spaces are universally closed over $S$ (see Theorem A).

Theorem 32. Let $I_{\eta}$ be a torsion-free, rank-1 sheaf on $X(\eta)$. Then, the following statements hold.

(1) There is an extension $\mathcal{I}$ of $I_{\eta}$.

(2) If $I_{\eta}$ is simple, then there is an extension $\mathcal{I}$ of $I_{\eta}$ that is simple over $S$.

(3) If $I_{\eta}$ is (simple and) semi-stable with respect to $\mathcal{E}(\eta)$, then there is an extension $\mathcal{I}$ of $I_{\eta}$ that is (simple and) semi-stable over $S$ with respect to $\mathcal{E}$. 
(4) Let $\sigma: S \rightarrow X$ be a section through the smooth locus of $X / S$. If $I_{\eta}$ is $\sigma(\eta)$ quasi-stable with respect to $\mathcal{E}(\eta)$, then there is an extension $\mathcal{I}$ of $I_{\eta}$ that is $\sigma$-quasi-stable over $S$ with respect to $\mathcal{E}$.

(5) If $I_{\eta}$ is quasi-stable with respect to $\mathcal{E}(\eta)$, then there is an extension $\mathcal{I}$ of $I_{\eta}$ that is quasi-stable over $S$ with respect to $\mathcal{E}$.

Proof. Statement (1) follows from [3, Lemma 7.8 (i), p. 100].

We prove (2) now. By (1), we may pick an extension $\mathcal{I}$ of $I_{\eta}$. If $\mathcal{I}(s)$ is simple, then we are done. If not, by Proposition 1, there is a non-empty, proper subcurve $Y \varsubsetneqq X(s)$ such that $\mathcal{I}(s)$ decomposes at $Y$. In this case, let

$$
\mathcal{I}^{1}:=\operatorname{ker}\left(\mathcal{I} \rightarrow \mathcal{I}(s)_{Y}\right) \quad \text { and } \quad \mathcal{I}^{-1}:=\operatorname{ker}\left(\mathcal{I} \rightarrow \mathcal{I}(s)_{Y^{c}}\right) .
$$

By Lemma 28, the set $C^{1}$ (resp. $C^{-1}$ ) of subcurves $Z \subseteq X(s)$ such that $\mathcal{I}^{1}(s)$ (resp. $\mathcal{I}^{-1}(s)$ ) decomposes at $Z$ is contained in the set $C$ of subcurves $Z \subseteq X(s)$ such that $\mathcal{I}(s)$ decomposes at $Z$. If $C^{1}$ (or $C^{-1}$ ) is strictly contained in $C$, then replace $\mathcal{I}$ by $\mathcal{I}^{1}$ (or $\mathcal{I}^{-1}$ ) and start the above procedure again, but now with a "better" extension. If not, then both $\mathcal{I}^{1}(s)$ and $\mathcal{I}^{-1}(s)$ decompose at $Y$. In this case, let

$$
\mathcal{I}^{2}:=\operatorname{ker}\left(\mathcal{I}^{1} \rightarrow \mathcal{I}^{1}(s)_{Y}\right) \text { and } \mathcal{I}^{-2}:=\operatorname{ker}\left(\mathcal{I}^{-1} \rightarrow \mathcal{I}_{Y^{c}}^{-1}\right),
$$

and apply the argument used above for $\mathcal{I}^{1}$ and $\mathcal{I}^{-1}$ to both $\mathcal{I}^{2}$ and $\mathcal{I}^{-2}$. Applying the above procedure repeatedly, we either obtain an extension $\mathcal{I}$ of $I_{\eta}$ that is simple over $S$, or we end up with two infinite filtrations of a certain extension $\mathcal{I}$,

$$
\begin{array}{r}
\cdots \subseteq \mathcal{I}^{i} \subseteq \cdots \subseteq \mathcal{I}^{1} \subseteq \mathcal{I}^{0}:=\mathcal{I}, \\
\cdots \subseteq \mathcal{I}^{-i} \subseteq \cdots \subseteq \mathcal{I}^{-1} \subseteq \mathcal{I}^{0}:=\mathcal{I},
\end{array}
$$

with quotients

$$
\frac{\mathcal{I}^{i}}{\mathcal{I}^{i+1}}=\mathcal{I}^{i}(s)_{Y} \text { and } \quad \frac{\mathcal{I}^{-i}}{\mathcal{I}^{-i-1}}=\mathcal{I}^{-i}(s)_{Y^{c}}
$$

for $i \geq 0$, where $Y \varsubsetneqq X(s)$ is a non-empty, proper subcurve such that $\mathcal{I}^{i}(s)$ decomposes at $Y$ for every integer $i$. We will show by contradiction that the latter situation is not possible. We may assume $R$ is complete. (If not, just extend the sheaves $\mathcal{I}^{i}$ over the completion of $R$.) By Lemma 24, there are $S$-flat quotients $\mathcal{F}$ and $\mathcal{G}$ of $\mathcal{I}$ such that $\mathcal{F}(s)=\mathcal{I}(s)_{Y}$ and $\mathcal{G}(s)=\mathcal{I}(s)_{Y^{c}}$. Consider the induced map $\phi: \mathcal{I} \rightarrow \mathcal{F} \oplus \mathcal{G}$. By assumption, $\phi(s)$ is an isomorphism. Since being an isomorphism is an open property, $\phi$ is an isomorphism. Thus, $I_{\eta} \cong \mathcal{I}(\eta)$ is not simple, a contradiction. The proof of (2) is complete.

We prove (3) now. By (1), we may pick an extension $\mathcal{I}$ of $I_{\eta}$. Consider the infinite filtration

$$
\cdots \subseteq \mathcal{I}^{i} \subseteq \cdots \subseteq \mathcal{I}^{1} \subseteq \mathcal{I}^{0}:=\mathcal{I}
$$

with quotients

$$
\frac{\mathcal{I}^{i}}{\mathcal{I}^{i+1}}=\mathcal{I}^{i}(s)_{Y_{i}},
$$

where $Y_{i} \subseteq X(s)$ is the maximum subcurve among the subcurves $W \subseteq X(s)$ with minimum $\beta_{\mathcal{I}^{i}(s)}(W)$, for each $i \geq 0$. We claim that $\mathcal{I}^{i}(s)$ is semi-stable with respect to $\mathcal{E}(s)$ for some $i \geq 0$. Suppose by contradiction that our claim is false. We may assume $R$ is complete. (If not, just extend $\mathcal{E}$ and the sheaves $\mathcal{I}^{i}$ over the completion 
of $R$.) Since $\mathcal{I}^{i}(s)$ is not semi-stable, $Y_{i}$ is a non-empty, proper subcurve of $X(s)$ with $\beta_{\mathcal{I}^{i}(s)}\left(Y_{i}\right)<0$, for every $i \geq 0$. By Lemma 30, up to replacing $\mathcal{I}$ by $\mathcal{I}^{j}$ for a certain $j$, we may assume that both $Y_{i}$ and $\beta_{\mathcal{I}^{i}(s)}\left(Y_{i}\right)$ do not depend on $i$, and $\mathcal{I}^{i}(s)$ decomposes at $Y_{i}$ for every $i \geq 0$. Let $Y:=Y_{i}$ and $\beta:=\beta_{\mathcal{I}^{i}(s)}\left(Y_{i}\right)$ for every $i \geq 0$. By Lemma 24, there is an $S$-flat quotient $\mathcal{F}$ of $\mathcal{I}$ such that $\mathcal{F}(s)=\mathcal{I}(s)_{Y}$. Since $\mathcal{F}$ is $S$-flat and $\chi(\mathcal{F}(s) \otimes \mathcal{E}(s))=r \beta<0$, also $\chi(\mathcal{F}(\eta) \otimes \mathcal{E}(\eta))<0$. Thus, $I_{\eta} \cong \mathcal{I}(\eta)$ is not semi-stable with respect to $\mathcal{E}(\eta)$, a contradiction. So, there is an extension $\mathcal{I}$ of $I_{\eta}$ that is semi-stable over $S$ with respect to $\mathcal{E}$. Suppose now that $I_{\eta}$ is simple. It is not necessarily true that $\mathcal{I}$ is simple over $S$. Nevertheless, we can apply the construction in the proof of (2) to $\mathcal{I}$, and get a simple sheaf on $X / S$ that will also be semi-stable with respect to $\mathcal{E}$ by Lemma 25 . The proof of (3) is complete.

We prove (4) now. By (3), there is a semi-stable sheaf $\mathcal{I}$ on $X / S$ with respect to $\mathcal{E}$ such that $\mathcal{I}(\eta) \cong I_{\eta}$. Consider the infinite filtration

$$
\cdots \subseteq \mathcal{I}^{i} \subseteq \cdots \subseteq \mathcal{I}^{1} \subseteq \mathcal{I}^{0}:=\mathcal{I}
$$

with quotients

$$
\frac{\mathcal{I}^{i}}{\mathcal{I}^{i+1}}=\mathcal{I}^{i}(s)_{Y_{i}},
$$

where $Y_{i} \subseteq X(s)$ is the minimum subcurve of $X(s)$ containing $\sigma(s)$ such that $\beta_{\mathcal{I}^{i}(s)}\left(Y_{i}\right)=0$, for $i \geq 0$. We claim that $\mathcal{I}^{i}(s)$ is $\sigma(s)$-quasi-stable with respect to $\mathcal{E}(s)$ for some $i \geq 0$. In fact, by Lemma 31 ,

$$
Y_{0} \subseteq Y_{1} \subseteq \cdots \subseteq Y_{i} \subseteq \cdots
$$

Thus, up to replacing $\mathcal{I}$ by $\mathcal{I}^{j}$ for a certain $j$, we may assume $Y_{i}$ does not depend on $i$. Let $Y:=Y_{i}$ for every $i \geq 0$. We will show that $Y=X(s)$. It follows from Lemma 31 that $\mathcal{I}^{i}(s)$ decomposes at $Y$ for every $i \geq 0$. We may now assume $R$ is complete. (If not, just extend $\sigma, \mathcal{E}$ and the sheaves $\mathcal{I}^{i}$ over the completion of $R$.) By Lemma 24, there is an $S$-flat quotient $\mathcal{F}$ of $\mathcal{I}$ such that $\mathcal{F}(s)=\mathcal{I}(s)_{Y}$. Since $\beta_{\mathcal{I}(s)}(Y)=0$, we have $\chi(\mathcal{F}(s) \otimes \mathcal{E}(s))=0$. Since $\mathcal{F}$ is $S$-flat, $\chi(\mathcal{F}(\eta) \otimes \mathcal{E}(\eta))=0$ as well. Since $\chi(\mathcal{F}(\eta) \otimes \mathcal{E}(\eta))=0$ and $\mathcal{I}(\eta)$ is semi-stable, $\mathcal{F}(\eta)$ is semi-stable (on a subcurve of $X(\eta)$ ). So, $\mathcal{F}$ is torsion-free on $X / S$. Since $\sigma$ factors through the $S$ smooth locus of $X$, the sheaf $\sigma^{*} \mathcal{F}$ is free. Since $\sigma^{*} \mathcal{F}(s) \neq 0$, also $\sigma^{*} \mathcal{F}(\eta) \neq 0$. Since $\mathcal{I}(\eta)$ is $\sigma(\eta)$-quasi-stable, $\mathcal{F}(\eta)=\mathcal{I}(\eta)$. By flatness, $\mathcal{F}(s)=\mathcal{I}(s)$. So, $Y=X(s)$, and thus $\mathcal{I}(s)$ is $\sigma(s)$-quasi-stable. The proof of (4) is complete.

The proof of (5) will be left to the reader. Roughly speaking, the proof consists of applying $n$ times the argument in the proof of (4), where $n$ is the number of irreducible components of $X(s)$.

Remark 33. Theorem 32 is not just an existence proof. In fact, we have established a method to produce an extension of $I_{\eta}$ with the same "good" properties (semistability, quasi-stability, etc.) as $I_{\eta}$, given any extension $\mathcal{I}$. We just construct a filtration

$$
\cdots \subseteq \mathcal{I}^{i} \subseteq \cdots \subseteq \mathcal{I}^{1} \subseteq \mathcal{I}^{0}=\mathcal{I}
$$

of $\mathcal{I}$ with quotients of the form

$$
\frac{\mathcal{I}^{i}}{\mathcal{I}^{i+1}}=\mathcal{I}^{i}(s)_{Y_{i}}
$$


where $Y_{i} \subseteq X(s)$ is a suitably chosen subcurve for each $i \geq 0$, as described in the proof of Theorem 32. Then, $\mathcal{I}^{i}$ will be a "good" extension of $I_{\eta}$ for some $i \geq 0$. Note, however, that the minimum such $i$ depends on the original extension $\mathcal{I}$.

\section{The FIne MOdULi SPACES}

Let $f: X \rightarrow S$ be a flat, projective map whose geometric fibers are curves. Let $\omega$ be a relative dualizing sheaf for $f$. Let $\mathbf{J}^{*}$ denote the contravariant functor from the category of locally Noetherian $S$-schemes to sets, defined on an $S$-scheme $T$ by

$$
\mathbf{J}^{*}(T):=\{\text { simple, torsion-free, rank-1 sheaves on } X \times T / T\} / \sim,
$$

where $\mathcal{I}_{1} \sim \mathcal{I}_{2}$ if there is an invertible sheaf $M$ on $T$ such that $\mathcal{I}_{1} \cong \mathcal{I}_{2} \otimes p^{*} M$, for $p: X \times T \rightarrow T$ the projection. Let $\mathbf{J}$ be the étale sheaf associated to $\mathbf{J}^{*}$. By [3. Thm. 7.4, p. 99], the functor $\mathbf{J}$ is represented by an algebraic space $J$, locally of finite type over $S$. Note that the formation of $J$ commutes with base change.

For every integer $d$, let $J_{d} \subseteq J$ be the subspace parameterizing simple, torsionfree, rank-1 sheaves $\mathcal{I}$ on $X / S$ with $\chi(\mathcal{I} / S)=d$. It is clear that $J_{d}$ is an open subspace of $J$, and that $J$ is the disjoint union of the $J_{d}$, for $d$ ranging through all the integers. The formation of $J_{d}$ commutes also with base change.

Fix an integer $d$. Fix a vector bundle $\mathcal{E}$ on $X$ of $\operatorname{rank} r$ and $\operatorname{deg}(\mathcal{E} / S)=-r d$. We consider $\mathcal{E}$ our polarization on $X / S$. Let $J_{\mathcal{E}}^{s}\left(\right.$ resp. $J_{\mathcal{E}}^{s s}$, resp. $J_{\mathcal{E}}^{q s}$ ) denote the subspace of $J_{d}$ parameterizing simple, torsion-free, rank-1 sheaves on $X / S$ that are stable (resp. semi-stable, resp. quasi-stable) with respect to $\mathcal{E}$. If $\sigma: S \rightarrow X$ is a section of $f$ through the $S$-smooth locus of $X$, let $J_{\mathcal{E}}^{\sigma}$ denote the subspace of $J_{d}$ parameterizing simple, torsion-free, rank- 1 sheaves on $X / S$ that are $\sigma$-quasi-stable with respect to $\mathcal{E}$. It is clear from the definitions in Section 1 that

$$
J_{\mathcal{E}}^{s} \subseteq J_{\mathcal{E}}^{\sigma} \subseteq J_{\mathcal{E}}^{q s} \subseteq J_{\mathcal{E}}^{s s} \subseteq J_{d}
$$

The formations of all the above spaces commute with base change.

Proposition 34. The subspaces $J_{\mathcal{E}}^{s}, J_{\mathcal{E}}^{\sigma}, J_{\mathcal{E}}^{q s}, J_{\mathcal{E}}^{s s} \subseteq J_{d}$ are open.

Proof. Let $T$ be an $S$-scheme and $\mathcal{I}$ a torsion-free, rank-1 sheaf on $X_{T} / T$ with $\chi(\mathcal{I} / T)=d$. Suppose there is $t \in T$ such that $\mathcal{I}(t)$ is semi-stable with respect to $\mathcal{E}(t)$. By Theorem 19, there are an étale map $h: V \rightarrow T$ containing $t$ in its image and a vector bundle $\mathcal{F}$ on $X_{V}$ of rank $2 r$ and $\operatorname{det} \mathcal{F} \cong\left(\operatorname{det} \mathcal{E}_{V}\right)^{\otimes 2}$ such that $R^{1} f_{V *}\left(\mathcal{I}_{V} \otimes \mathcal{F}\right)=0$. Let $U:=h(V)$. Since $h$ is étale, $U \subseteq T$ is open. By Theorem $19, \mathcal{I}_{U}$ is semi-stable on $X_{U} / U$ with respect to $\mathcal{E}_{U}$. So $J_{\mathcal{E}}^{s s} \subseteq J_{d}$ is open.

In the same way, we may use Theorems 20,21 and 22 to show that the remaining subspaces in the statement are open.

Proof of Theorem A. We show first that $J_{\mathcal{E}}^{s s}$ is of finite type over $S$. We may assume $S$ is Noetherian. Fix an ample sheaf $\mathcal{O}_{X}(1)$ on $X / S$. For every integer $m$, let $\Sigma_{m} \subseteq J_{\mathcal{E}}^{s s}$ denote the open subspace parameterizing $m$-regular sheaves on $X / S$ with respect to $\mathcal{O}_{X}(1)$. The subspaces $\Sigma_{m}$ cover $\bar{J}_{\mathcal{E}}^{s s}$ and are of finite type over $S$ [3, Lemma 7.3 and Thm. 7.4 , pp. $98-99]$. So, we need only show that $\Sigma_{m}=\bar{J}_{\mathcal{E}}^{s s}$ for some $m$.

Since $S$ is Noetherian, there is an integer $M$ such that $\chi\left(\omega(k)_{Y}\right) \leq M$ and $\left.\operatorname{deg} \mathcal{E}(k)\right|_{Y} \leq M$ for every $s \in S$, every field extension $k \supseteq k(s)$ and every subcurve $Y \subseteq X(k)$. Let $s \in S$, and consider a field extension $k \supseteq k(s)$ such that $C:=X(k)$ 
has geometrically integral irreducible components. Let $I$ be a semi-stable sheaf on $C$ with respect to $E:=\mathcal{E}(k)$. For every integer $m$ and every subcurve $Y \subseteq C$,

$$
\chi\left(I(m)_{Y}\right)=\chi\left(I_{Y}\right)+\left.m \operatorname{deg} \mathcal{O}_{C}(1)\right|_{Y} \geq-\left(\left.\operatorname{deg} E\right|_{Y}\right) / r+m \geq-M / r+m .
$$

If $m>M(r+1) / r$, then $\chi\left(I(m)_{Y}\right)>\chi\left(\omega(k)_{Y}\right)$ for every subcurve $Y \subseteq C$. By duality, $h^{1}(C, I(m))=0$, and hence $I$ is $m$-regular with respect to $\mathcal{O}_{C}(1)$. Therefore, $\Sigma_{m}=\bar{J}_{\mathcal{E}}^{s s}$ for every $m>M(r+1) / r$.

Since $J_{\mathcal{E}}^{s s}$ is of finite type over $S$, statement (1) follows from Theorem 32 , statement (2) follows from Proposition 26, and statement (3) follows from Proposition 27 and Theorem 32 .

Lemma 35. Assume there are sections $\sigma_{1}, \ldots, \sigma_{n}: S \rightarrow X$ of $f$ such that

(1) $\sigma_{i}$ factors through the $S$-smooth locus of $X$ for $i=1, \ldots, n$;

(2) for every $s \in S$, every irreducible component of $X(s)$ is geometrically integral and contains $\sigma_{i}(s)$ for some $i$.

Then, there are polarizations $\mathcal{E}_{j}$ on $X / S$ such that $J=\bigcup_{j} J_{\mathcal{E}_{j}}^{s}$.

Proof. For each $n$-uple of integers $\underline{e}:=\left(e_{1}, \ldots, e_{n}\right)$ and each integer $r>0$ such that $n r \mid\left(e_{1}+\cdots+e_{n}\right)$, let

$$
\mathcal{E}_{(\underline{e}, r)}:=\mathcal{O}_{X}\left(-e_{1} \sigma_{1}(S)\right) \oplus \cdots \oplus \mathcal{O}_{X}\left(-e_{n} \sigma_{n}(S)\right) \oplus \mathcal{O}_{X}^{\oplus n(r-1)} .
$$

For every $s \in S$, varying $\underline{e}$ and $r$, the multi-slopes of $\mathcal{E}_{(\underline{e}, r)}(s)$ cover all $n_{s}$-uples of rational numbers, where $n_{s}$ is the number of irreducible components of $X(s)$. By Corollary 15,

$$
J=\bigcup_{(\underline{e}, r)} J_{\mathcal{E}_{(\underline{e}, r)}}^{s} .
$$

Lemma 36. Let $\Sigma \subseteq J$ be an open subspace of finite type over $S$. Then, there is an étale surjection $U \rightarrow S$ such that $\Sigma \times U$ is a scheme.

Proof. We may assume $S$ is Noetherian. By Lemmas 18 and 35, replacing $S$ by an étale covering, we may assume there are a section $\sigma: S \rightarrow X$ of $f$ through the $S$-smooth locus of $X$ and polarizations $\mathcal{E}_{j}$ on $X / S$ such that $J=\bigcup_{j} J_{\mathcal{E}_{j}}^{\sigma}$. Since $\Sigma$ is of finite type over $S$, hence Noetherian, we need only show that for every polarization $\mathcal{E}$ on $X / S$ and every $s \in S$ there is an étale map $U \rightarrow S$ containing $s$ in its image, and such that $J_{\mathcal{E}}^{\sigma} \times U$ is a scheme.

Fix a polarization $\mathcal{E}$ on $X / S$ and a point $s \in S$. Let $r$ be the rank of $\mathcal{E}$. Let $T \rightarrow J_{\mathcal{E}}^{\sigma}$ be an étale surjection such that $T$ is a scheme and $X \times T$ admits a universal torsion-free, rank-1 sheaf $\mathcal{I}$ over $T$. Now, $T(s)$ is Noetherian since $J_{\mathcal{E}}^{\sigma}$ is of finite type over $S$ by Theorem A. It follows from Theorem 20 that there are étale maps $U \rightarrow S$ and $V \rightarrow T \times U$, a point $u \in U$ lying over $s$ and vector bundles $\mathcal{F}_{1}, \ldots, \mathcal{F}_{n}$ on $X_{U}$ of rank $2 r$ and $\operatorname{det} \mathcal{F}_{i} \cong\left(\operatorname{det} \mathcal{E}_{U}\right)^{\otimes 2} \otimes \mathcal{O}_{X_{U}}(-\sigma(S) \times U)$ such that

(1) the image of $V \rightarrow T \times U$ contains $T(u)$;

(2) $f_{V *}\left(\mathcal{I}_{V} \otimes \mathcal{F}_{i, V}\right)=0$ and $R^{1} f_{V *}\left(\mathcal{I}_{V} \otimes \mathcal{F}_{i, V}\right)$ is invertible for each $i$;

(3) the natural map

$$
\mathcal{I}_{V} \longrightarrow \mathcal{F}_{i, V}^{*} \otimes \omega_{V} \otimes f_{V}^{*} R^{1} f_{V *}\left(\mathcal{I}_{V} \otimes \mathcal{F}_{i, V}\right)
$$

is injective with $V$-flat cokernel for each $i$. 
For each $i=1, \ldots, n$ let $W_{i} \subseteq \operatorname{Quot}_{\mathcal{F}_{i}^{*} \otimes \omega_{U}\left|X_{U}\right| U}$ be the open subscheme whose $B$ points, for each $U$-scheme $B$, correspond to quotients $q: \mathcal{F}_{i, B}^{*} \otimes \omega_{B} \rightarrow \mathcal{Q}$ such that $\mathcal{J}_{q}:=\operatorname{ker}(q)$ is semi-stable, $f_{B *}\left(\mathcal{J}_{q} \otimes \mathcal{F}_{i, B}\right)=0$ and $R^{1} f_{B *}\left(\mathcal{J}_{q} \otimes \mathcal{F}_{i, B}\right)$ is invertible. (In fact, if $q$ corresponds to a $B$-point of $W_{i}$ then $R^{1} f_{B *}\left(\mathcal{J}_{q} \otimes \mathcal{F}_{i, B}\right) \cong \mathcal{O}_{B}$.) There's a natural map $W_{i} \rightarrow J_{\mathcal{E}}^{s s} \times U$, defined by sending $q$ to $\mathcal{J}_{q}$. By Theorem 20 , this map factors through $J_{\mathcal{E}}^{\sigma} \times U$. Moreover, this map is clearly an open embedding.

Now, the $W_{i}$ cover $J_{\mathcal{E}}^{s s}(u)$ because of (1)-(3) above. Since $J_{\mathcal{E}}^{\sigma}$ is proper over $S$ by Theorem A, up to replacing $U$ by an open neighborhood of $u$, we may assume that $J_{\mathcal{E}}^{\sigma} \times U=\bigcup_{i} W_{i}$. Since $W_{1}, \ldots, W_{n}$ are schemes, so is $J_{\mathcal{E}}^{\sigma} \times U$.

In Subsection 7.1 we will drop the hypothesis that $\Sigma$ is of finite type over $S$. More precisely, Corollary 52 asserts that Lemma 36 holds for $J$ in place of $\Sigma$.

\section{Certain special Cases}

Let $X$ be a connected curve over an algebraically closed field $k$. Let $g$ denote the arithmetic genus of $X$.

Let $J$ denote the algebraic space parameterizing simple, torsion-free, rank-1 sheaves on $X$. By Lemma 35, there are enough polarizations $E_{j}$ on $X$ such that $J=\bigcup_{j} J_{E_{j}}^{s}$. By Theorem A, the $J_{E_{j}}^{s}$ are of finite type over $k$. By Lemma 36, the $J_{E_{j}}^{s}$ are schemes. So, $J$ is a scheme locally of finite type over $k$.

Example 37 (Joining two curves). Assume there are subcurves $Y, Z \subseteq X$ covering $X$ such that $Y$ and $Z$ intersect transversally at a unique point, and this point is smooth on $Y$ and on $Z$. Let $J_{X}$ (resp. $J_{Y}$, resp. $J_{Z}$ ) denote the scheme parameterizing simple, torsion-free, rank-1 sheaves on $X$ (resp. $Y$, resp. $Z$ ). Since every simple, torsion-free, rank-1 sheaf on $X$ must be invertible along $Y \cap Z$, we have a map $J_{X} \rightarrow J_{Y} \times J_{Z}$, defined by restriction of sheaves on $X$ to $Y$ and $Z$. It can be shown that the above map is an isomorphism.

Example 38 (Abel maps). Let

$$
\delta_{X}:=\min _{Y \varsubsetneqq X} \chi\left(\mathcal{O}_{Y \cap Y^{c}}\right),
$$

where $Y$ runs through all non-empty, proper subcurves of $X$. (If $X$ is irreducible, let $\delta_{X}:=\infty$.) Since $X$ is connected, $\delta_{X}>0$.

Let $I \subseteq \mathcal{O}_{X}$ be the ideal sheaf of a subscheme $D \subset X$ of finite length $m$. For each non-empty, proper subcurve $Y \varsubsetneqq X$, the natural commutative diagram

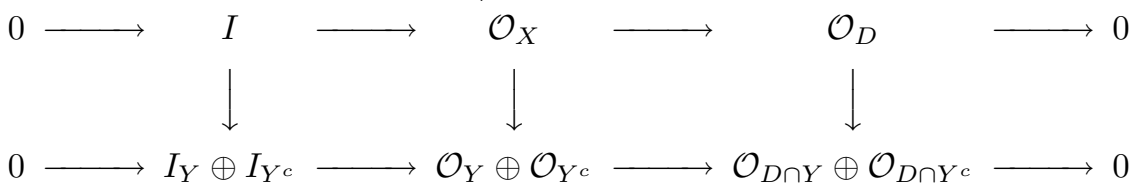

has exact horizontal sequences. By the snake lemma,

$$
\chi\left(I_{Y}\right)+\chi\left(I_{Y^{c}}\right)-\chi(I) \geq \chi\left(\mathcal{O}_{Y \cap Y^{c}}\right)-m,
$$

with equality if and only if $D \subseteq Y \cap Y^{c}$. Therefore, if $m<\delta_{X}$, then $I$ is simple. On the other hand, if $D=Y \cap Y^{c}$ for a non-empty, proper subcurve $Y \varsubsetneqq X$, then $I$ is not simple. To summarize, there are subschemes $D \subset X$ of length $\delta_{X}$ whose ideal sheaves are not simple, and $\delta_{X}$ is the minimum length where this occurs.

Let $m$ be any integer with $0 \leq m<\delta_{X}$. Let $H_{m}$ denote the Hilbert scheme of $X$, parameterizing length- $m$ subschemes of $X$. Of course, $H_{1}=X$. Let $M$ be an 
invertible sheaf on $X$. For every subscheme $D \subseteq X$, let $\mathcal{I}_{D} \subseteq \mathcal{O}_{X}$ denote its ideal sheaf. Since $m<\delta_{X}$, we have a well-defined map

$$
\begin{aligned}
\alpha_{M}^{m}: H_{m} & \rightarrow \quad J_{d} \\
{[D] } & \mapsto\left[\mathcal{I}_{D} \otimes M\right]
\end{aligned}
$$

where $d:=\chi(M)-m$. The map $\alpha_{M}^{m}$ is called an Abel map in degree $m$ of $X$. If $\delta_{X}>1$, let $\alpha_{M}:=\alpha_{M}^{1}$. If $X$ is irreducible, then $\alpha_{M}$ is a closed embedding [3] Thm. 8.8, p. 108]. In addition, if $g=1$, then $\alpha_{M}$ is an isomorphism [3, Ex. 8.9, p. 109]. In general, I do not know whether $\alpha_{M}$ is a closed embedding, but the case $g=1$ will be treated below.

Example 39 (Curves with genus 1). Use the notation in Example 38. Assume that $g=1$ and $\delta_{X}>1$. We claim first that every non-empty, connected, proper subcurve of $X$ has arithmetic genus 0 . In fact, if $Y \subseteq X$ is a subcurve, then $h^{1}\left(Y, \mathcal{O}_{Y}\right) \leq 1$. Assume equality. Assume as well that $Y$ is connected. Let $Z:=Y^{c}$. Then $h^{1}\left(Z, \mathcal{O}_{Z}\right)=0$ and $Z$ has exactly $\chi\left(\mathcal{O}_{Y \cap Z}\right)$ connected components. Since $X$ is connected, $\chi\left(\mathcal{O}_{W \cap W^{c}}\right)=\chi\left(\mathcal{O}_{W \cap Y}\right)=1$ for each connected component $W \subseteq Z$. Since $\delta_{X}>1$, we have $Y=X$, proving our claim.

We claim second that $\mathcal{O}_{X}$ is the dualizing sheaf on $X$. In fact, since $g=1$, there is a non-zero map $h: \mathcal{O}_{X} \rightarrow \omega$, where $\omega$ is the dualizing sheaf on $X$. Let $Y \subseteq X$ be the non-empty subcurve such that $\mathcal{O}_{Y} \cong \operatorname{im}(h)$ and put $Z:=Y^{c}$. Since the map $\mathcal{O}_{Z} \rightarrow \omega_{Z}$ induced by $h$ is zero, $h$ factors through $\Omega \subseteq \omega$, where $\Omega$ is the dualizing sheaf on $Y$. So, $h^{1}\left(Y, \mathcal{O}_{Y}\right) \geq 1$. Hence there is a connected component of $Y$ with arithmetic genus 1. By our first claim, $Y=X$. Since $\chi(\omega)=\chi\left(\mathcal{O}_{X}\right)=0$ and $h$ is injective, $h$ is an isomorphism, proving our second claim.

Let $d$ be an integer and $M$ an invertible sheaf on $X$ of degree $d+1$. Let $J_{M} \subseteq J_{d}$ denote the subset parameterizing simple, torsion-free, rank-1 sheaves $I$ on $X$ such that $\chi\left(I_{Y}\right) \geq\left.\operatorname{deg} M\right|_{Y}$ for every non-empty, proper subcurve $Y \varsubsetneqq X$.

We claim that $J_{M}$ is a complete, open subscheme of $J_{d}$, and $\alpha_{M}$ factors through $J_{M}$. Indeed, let $p \in X$ be any non-singular point, and put $E:=M^{*} \otimes \mathcal{O}_{X}(p)$. By definition, a torsion-free, rank-1 sheaf $I$ on $X$ with $\chi(I)=d$ is $p$-quasi-stable with respect to $E$ if and only if $\chi\left(I_{Y}\right) \geq\left.\operatorname{deg} M\right|_{Y}$ for every non-empty, proper subcurve $Y \varsubsetneqq X$. Thus, $J_{M}=J_{E}^{p}$. It follows from Theorem A and Proposition 34 that $J_{M}$ is a complete, open subscheme of $J_{d}$.

In addition, let $q \in X$ and $Y \varsubsetneqq X$ be a connected, proper subcurve. Since the arithmetic genus of $Y$ is 0 , we have $\chi\left(\mathcal{I}_{q, Y}\right)=1$ if $q \notin Y$, and $\chi\left(\mathcal{M}_{q, Y}\right)=0$ otherwise. At any rate, we have $\chi\left(I_{Y}\right) \geq\left.\operatorname{deg} M\right|_{Y}$, where $I:=\mathcal{I}_{q} \otimes M$. So, $\alpha_{M}$ factors through $J_{M}$, proving our third claim.

We will show now that $\alpha_{M}$ is an isomorphism onto $J_{M}$. Indeed, we will construct the inverse map $\beta_{M}: J_{M} \rightarrow X$ as follows. Let $I$ be a simple, torsion-free, rank-1 sheaf on $X$ such that $\chi(I)=d$ and

$$
\chi\left(I_{Y}\right) \geq\left.\operatorname{deg} M\right|_{Y}
$$

for every non-empty, proper subcurve $Y \varsubsetneqq X$. We claim that

$$
h^{0}\left(X, I \otimes M^{*}\right)=0,
$$


and the unique (modulo $k^{*}$ ) non-zero map $\lambda: I \otimes M^{*} \rightarrow \mathcal{O}_{X}$ is an isomorphism onto the ideal sheaf $\mathcal{I}_{q}$ of a point $q \in X$. If so, let $q$ be the image under $\beta_{M}$ of the point of $J_{M}$ represented by $I$. If defined, $\beta_{M}$ is clearly the inverse to $\alpha_{M}$.

To show (39.2), let $\mu: \mathcal{O}_{X} \rightarrow I \otimes M^{*}$ be a map. Let $Y \subseteq X$ be the subcurve such that $\mathcal{O}_{Y} \cong \operatorname{im}(\mu)$. Then, $\mu$ factors through $J \otimes M^{*}$, for $J:=\operatorname{ker}\left(I \rightarrow I_{Y^{c}}\right)$. Suppose that $Y$ is non-empty. Since $\chi\left(I \otimes M^{*}\right)=-1$, it follows from (39.1) that $\chi\left(J \otimes M^{*}\right) \leq-1$. Since $\mu$ induces an injection $\mathcal{O}_{Y} \hookrightarrow J \otimes M^{*}$, we have $\chi\left(\mathcal{O}_{Y}\right) \leq-1$ as well. On the other hand, $h^{1}\left(Y, \mathcal{O}_{Y}\right) \leq 1$ because $g=1$, and hence $\chi\left(\mathcal{O}_{Y}\right) \geq 0$, reaching a contradiction. Thus $\mu=0$, proving (39.2).

Since $\mathcal{O}_{X}$ is the dualizing sheaf on $X$ by our second claim, and $\chi\left(I \otimes M^{*}\right)=-1$, it follows from (39.2) and duality that there is a unique (modulo $k^{*}$ ) non-zero map $\lambda: I \otimes M^{*} \rightarrow \mathcal{O}_{X}$. Let $Y \subseteq X$ be the subcurve such that $I_{Y} \otimes M^{*} \cong \operatorname{im}(\lambda)$. Since $Y$ is non-empty, it follows from our first claim that $h^{1}\left(Y^{c}, \mathcal{O}_{Y^{c}}\right)=0$. So, the ideal sheaf $\mathcal{I}_{Y^{c}}$ of $Y^{c}$ satisfies $\chi\left(\mathcal{I}_{Y^{c}}\right) \leq 0$, with equality only if $Y^{c}=\emptyset$. In addition, by (39.1), $\chi\left(I_{Y} \otimes M^{*}\right) \geq-1$ with equality only if $Y=X$. On the other hand, since there is an injection $I_{Y} \otimes M^{*} \hookrightarrow \mathcal{I}_{Y^{c}}$, we have $\chi\left(\mathcal{I}_{Y^{c}}\right) \geq \chi\left(I_{Y} \otimes M^{*}\right)$. Hence $Y=X$ or, in other words, $\lambda$ is injective. Since $\chi\left(I \otimes M^{*}\right)=-1$, the image of $\lambda$ is the ideal sheaf of a point, finishing the proof of our last claim.

Observe that we have naively defined $\beta_{M}$ as a map of sets, but it is clearly possible to apply the above argument to a family of torsion-free, rank-1 sheaves on $X$, and thus define $\beta_{M}$ as a map of schemes.

Example 40 (Locally planar curves). Assume $X$ is locally planar. In other words, assume $X$ can be embedded into a smooth surface. Then, Altman, Kleiman and Iarrobino [2, Cor. 7, p. 7] showed that the Hilbert scheme $H_{m}$ of $X$, parameterizing length- $m$ subschemes of $X$, is $m$-dimensional, reduced and a local complete intersection. In addition, the proof of 2 , Thm. 9, p. 8] can be easily adapted to show that $J$ is reduced, a local complete intersection, and has pure dimension $g$ at every point. Finally, it follows from [2, Thm. 5, p. 5] that the invertible sheaves form a dense, open subscheme of $J$.

Example 41 (Two-component curves). Assume $X$ has only two irreducible components, $X_{1}$ and $X_{2}$. Let $\delta$ denote the length of $X_{1} \cap X_{2}$. Let $P$ denote the Jacobian of $X$. Let $E$ be a polarization on $X$ of rank $r$ and degree $-r d$, for an integer $d$. For $i=1,2$, let $e_{i}:=-\left.\operatorname{deg} E\right|_{X_{i}}$ and $J_{E}^{i}$ denote the moduli space of $X_{i}$-quasi-stable sheaves on $X$ with respect to $E$. There are two cases.

(1) $r \nmid e_{1}$ : In this case, $J_{E}^{s}=J_{E}^{s s}$, and $J_{E}^{s}$ is complete. A torsion-free, rank-1 sheaf $I$ on $X$ is stable only if $-e_{i} / r<\chi\left(I_{X_{i}}\right)<-e_{i} / r+\delta$ for $i=1,2$. If $I$ is invertible, then the converse holds. Since there are $\delta$ integers in the interval $\left[-e_{i} / r,-e_{i} / r+\delta\right]$, there are exactly $\delta$ connected components of $P$ contained in $J_{\mathcal{E}}^{s}$. If $X$ is locally planar, then $P$ is dense in $J$, and thus $J_{E}^{s}$ has exactly $\delta$ irreducible components.

(2) $r \mid e_{1}$ : In this case, $J_{E}^{s} \varsubsetneqq J_{E}^{1}, J_{E}^{2} \varsubsetneqq J_{E}^{s s}$. Reasoning as in Case 1 , if $X$ is locally planar, then $J_{E}^{s s}$ has $\delta+1$ irreducible components, whereas $J_{E}^{1}$ and $J_{E}^{2}$ have $\delta$ components each, and $J_{E}^{s}$ has $\delta-1$ components. As we observed in Example 5 , we have $J_{E}^{q s}=J_{E}^{s s}$.

Case 1 corresponds to Caporaso's general case [7] 7.3, p. 646], whereas Case 2 corresponds to her special case. 
Assume now that $X_{1}$ and $X_{2}$ are smooth, and intersect at two ordinary nodes. So, $\delta=2$ and $X$ is locally planar. Let $P_{d+1} \subset P$ be the open subscheme parameterizing invertible sheaves of Euler characteristic $d+1$, and consider the (well-defined) map:

$$
\begin{aligned}
\alpha: X \times P_{d+1} & \rightarrow \quad J_{d} \\
(q, M) & \mapsto\left[\mathcal{I}_{q} \otimes M\right]
\end{aligned}
$$

One can show that $\alpha$ is surjective, and smooth with relative dimension 1 (cf. [11). In Case 1, we have that $J_{E}^{s}$ is the image under $\alpha$ of a connected component of $X \times P_{d+1}$. In Case 2, we have that $J_{E}^{s s}=J_{E}^{1} \cup J_{E}^{2}$, and both $J_{E}^{1}$ and $J_{E}^{2}$ are images under $\alpha$ of different connected components of $X \times P_{d+1}$. In fact, $J_{E}^{i}=\alpha\left(X \times P_{E}^{i}\right)$, where $P_{E}^{i}$ is the connected component of $P_{d+1}$ parameterizing invertible sheaves on $X$ with Euler characteristic $e_{i} / r+2$ on $X_{i}$, for $i=1,2$. The patching of $J_{E}^{1}$ and $J_{E}^{2}$ to produce $J_{E}^{s s}$ occurs on $J_{E}^{s}$, which is the image under $\alpha$ of both $\left(X_{1} \backslash X_{2}\right) \times P_{E}^{1}$ and $\left(X_{2} \backslash X_{1}\right) \times P_{E}^{2}$.

We observe the difference between our description and Caporaso's in loc. cit. As we are dealing with fine moduli spaces, without making identifications to guarantee separatedness, our moduli spaces $J_{E}^{s s}$ in Case 2 have more components than Caporaso's. In fact, to reach Caporaso's spaces, we have to identify and collapse the two non-stable components of $J_{E}^{s s}$ through the JH-equivalence into a locus of positive codimension. Such identification will be carried out in Section 7 by means of theta functions introduced in the next section.

\section{Theta FunCtions}

6.1. The determinant of cohomology. Let $f: X \rightarrow S$ be a flat, projective map whose geometric fibers are curves.

Construction 42. Let $\mathcal{E}$ be an $S$-flat, coherent sheaf on $X$. Assume there are complexes

$$
0 \rightarrow F^{0} \stackrel{\lambda}{\rightarrow} F^{1} \rightarrow 0 \text { and } 0 \rightarrow G^{0} \stackrel{\mu}{\rightarrow} G^{1} \rightarrow 0
$$

of free sheaves on $S$ of finite rank, and quasi-isomorphisms $p: F^{\cdot} \rightarrow R f_{*} \mathcal{E}$ and $q: G \cdot \rightarrow R f_{*} \mathcal{E}$. By [20] Prop. 4, p. 39], there is a unique quasi-isomorphism $g: F^{\cdot} \rightarrow G$ such that $p=q g$ (up to homotopy). Consider the commutative diagram of maps

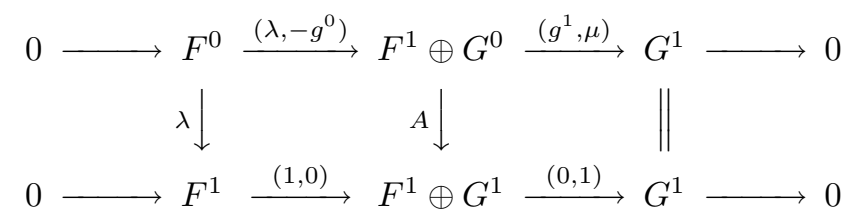

where

$$
A:=\left[\begin{array}{cc}
1 & 0 \\
g^{1} & \mu
\end{array}\right] .
$$

Since $g$ is a quasi-isomorphism, the upper sequence is exact. Thus, taking its determinant, we get an isomorphism $d(g): \operatorname{det} F \rightarrow \operatorname{det} G$, where

$$
\operatorname{det} F^{\cdot}:=\operatorname{det} F^{1} \otimes\left(\operatorname{det} F^{0}\right)^{-1} \text { and } \operatorname{det} G^{\cdot}:=\operatorname{det} G^{1} \otimes\left(\operatorname{det} G^{0}\right)^{-1} \text {. }
$$


(Here and wherever needed we use implicitly the unadjusted interchanging isomorphism $L \otimes M \cong M \otimes L$, sending $\sigma \otimes \tau$ to $\tau \otimes \sigma$.)

If $g_{1}: F^{\cdot} \rightarrow G^{\cdot}$ is another quasi-isomorphism with $p=q g_{1}$, then $g_{1}$ is homotopic to $g$. In other words, there is a map $\iota: F^{1} \rightarrow G^{0}$ such that $g_{1}^{0}-g^{0}=\iota \lambda$ and $g_{1}^{1}-g^{1}=\mu \iota$. The homotopy gives rise to a commutative diagram of maps

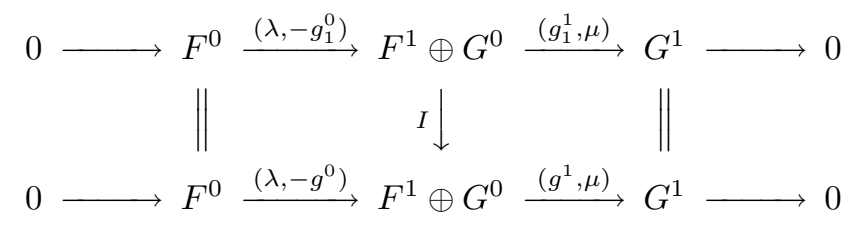

where

$$
I:=\left[\begin{array}{ll}
1 & 0 \\
\iota & 1
\end{array}\right]
$$

Since $I$ has determinant 1 , the determinant of the first row is equal to that of the second row. So, $d(g)$ depends only on the homotopy class of $g$. Let $d(p, q):=d(g)$.

Let

$$
0 \rightarrow H^{0} \stackrel{\nu}{\rightarrow} H^{1} \rightarrow 0
$$

be another complex of free sheaves on $S$ of finite rank, and $r: H^{\cdot} \rightarrow R f_{*} \mathcal{E}$ a quasi-isomorphism. Let $h: G^{\cdot} \rightarrow H^{\cdot}$ be a quasi-isomorphism such that $q=r h$ (up to homotopy). Consider the commutative diagram of exact sequences

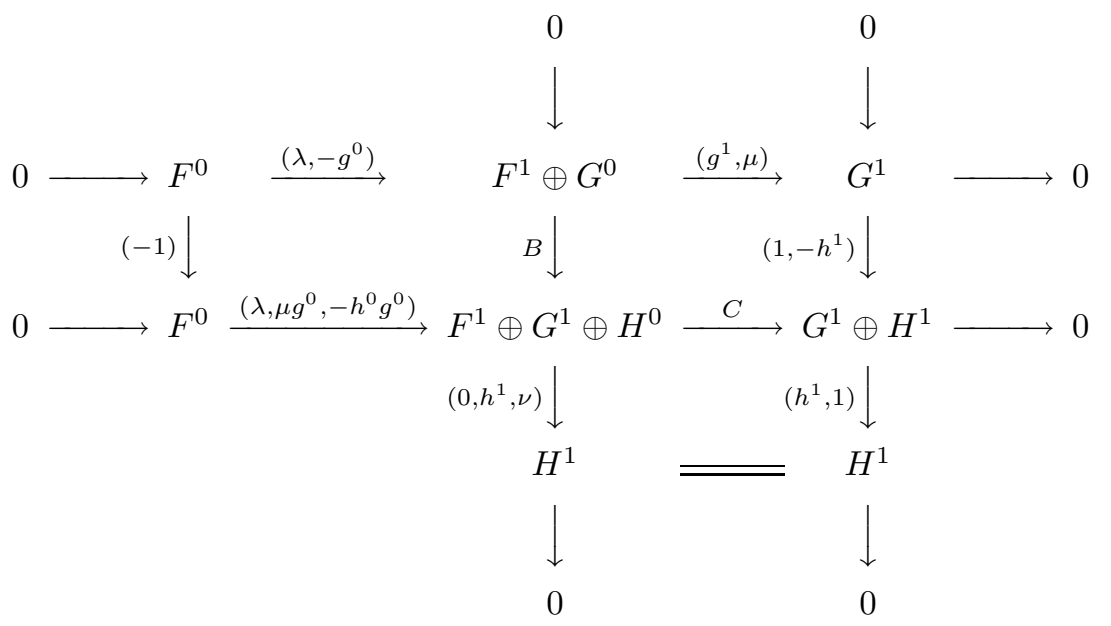

where

$$
B:=\left[\begin{array}{cc}
-1 & 0 \\
0 & \mu \\
0 & -h^{0}
\end{array}\right] \quad \text { and } \quad C:=\left[\begin{array}{ccc}
-g^{1} & 1 & 0 \\
h^{1} g^{1} & 0 & \nu
\end{array}\right]
$$

Taking determinants, and using that

$$
\operatorname{rk}\left(F^{0}\right)-\operatorname{rk}\left(F^{1}\right)=\operatorname{rk}\left(G^{0}\right)-\operatorname{rk}\left(G^{1}\right)=\operatorname{rk}\left(H^{0}\right)-\operatorname{rk}\left(H^{1}\right)=\chi(\mathcal{E} / S),
$$

we have $d(h g)=(-1)^{\operatorname{rk}\left(G^{0}\right)\left(\operatorname{rk}\left(G^{1}\right)+1\right)} d(h) d(g)$.

If $\chi(\mathcal{E} / S)=0$, then $\operatorname{rk}\left(F^{0}\right)=\operatorname{rk}\left(F^{1}\right)$ and $\operatorname{rk}\left(G^{0}\right)=\operatorname{rk}\left(G^{1}\right)$. Taking determinants in $(42.1)$, we get $\operatorname{det} \mu=d(g) \operatorname{det} \lambda$. In addition, $d(h g)=d(h) d(g)$. 
We will employ the above construction in a patching construction of the determinant of cohomology, after the next observation.

Observation 43. By [18, Exp. 3, Prop. 4.8, p. 257], the complex $R \cdot f_{*} \mathcal{E}$ is perfect, i.e., there is an open covering $S=\bigcup_{\alpha} U_{\alpha}$ such that $\left.R \cdot f_{*} \mathcal{E}\right|_{U_{\alpha}}$ is quasi-isomorphic to a complex of free sheaves for every $\alpha$. Alternatively, we may prove $R f_{*} \mathcal{E}$ is perfect as follows. Let $\mathcal{O}_{X}(1)$ be an ample sheaf on $X / S$. Let $s \in S$. If $m>>0$, there is a section $h_{s} \in H^{0}\left(X(s), \mathcal{O}_{X}(m)(s)\right)$ which is nowhere a zero-divisor of $\mathcal{E}(s)$. In addition, up to replacing $S$ by a neighborhood of $s$, we may assume $R^{1} f_{*} \mathcal{E}(m)=0$, and that $h_{s}$ extends to a section $h \in H^{0}\left(X, \mathcal{O}_{X}(m)\right)$ such that the induced map $\mathcal{E} \stackrel{\cdot h}{\longrightarrow} \mathcal{E}(m)$ is injective with $S$-flat cokernel $\mathcal{C}$. Since the fibers of $\mathcal{C}$ have finite length, $R^{1} f_{*} \mathcal{C}=0$. Since $\mathcal{E}(m)$ and $\mathcal{C}$ are acyclic for $f_{*}$,

$$
0 \rightarrow f_{*} \mathcal{E}(m) \rightarrow f_{*} \mathcal{C} \rightarrow 0
$$

is a complex of locally free sheaves on $S$ that is quasi-isomorphic to $R f_{*} \mathcal{E}$.

Consider the collection $\Xi:=\Xi(\mathcal{E})$ of all triples $\alpha:=\left(U_{\alpha}, F_{\alpha}, p_{\alpha}\right)$, where $U \subseteq S$ is an open subscheme,

$$
0 \rightarrow F_{\alpha}^{0} \stackrel{\lambda_{\alpha}}{\longrightarrow} F_{\alpha}^{1} \rightarrow 0
$$

is a complex of free sheaves on $U_{\alpha}$ of finite rank, and $p_{\alpha}:\left.F_{\alpha}^{\cdot} \rightarrow R f_{*} \mathcal{E}\right|_{U_{\alpha}}$ is a quasi-isomorphism. By Construction 42 , if $\alpha, \beta \in \Xi$, the quasi-isomorphisms $\left.p_{\alpha}\right|_{U_{\alpha, \beta}}$ and $\left.p_{\beta}\right|_{U_{\alpha, \beta}}$ induce an isomorphism

$$
d_{\alpha, \beta}:=(-1)^{\mathrm{rk}\left(F_{\alpha}^{0}\right)\left(\operatorname{rk}\left(F_{\alpha}^{1}\right)+1\right)} d\left(\left.p_{\alpha}\right|_{U_{\alpha, \beta}},\left.p_{\beta}\right|_{U_{\alpha, \beta}}\right),
$$

where $U_{\alpha, \beta}:=U_{\alpha} \cap U_{\beta}$. By Construction 42 , if $\alpha, \beta, \gamma \in \Xi$,

$$
\left.d_{\alpha, \gamma}\right|_{U_{\alpha, \beta, \gamma}}=\left.\left.d_{\beta, \gamma}\right|_{U_{\alpha, \beta, \gamma}} d_{\alpha, \beta}\right|_{U_{\alpha, \beta, \gamma}}
$$

where $U_{\alpha, \beta, \gamma}:=U_{\alpha} \cap U_{\beta} \cap U_{\gamma}$. Since $S=\bigcup_{\alpha} U_{\alpha}$ by Observation 43, and the cocycle condition is met, there are an invertible sheaf $\mathcal{D}_{f}(\mathcal{E})$ on $S$ and isomorphisms $\zeta_{\alpha}:\left.\mathcal{D}_{f}(\mathcal{E})\right|_{U_{\alpha}} \cong \operatorname{det} F_{\alpha}$ for all $\alpha \in \Xi$, such that $\left.\zeta_{\beta}\right|_{U_{\alpha, \beta}}=\left.d_{\alpha, \beta} \zeta_{\alpha}\right|_{U_{\alpha, \beta}}$ for all $\alpha, \beta \in \Xi$.

If $\chi(\mathcal{E} / S)=0$, then $\left.\operatorname{det} \lambda_{\beta}\right|_{U_{\alpha, \beta}}=\left.d_{\alpha, \beta} \operatorname{det} \lambda_{\alpha}\right|_{U_{\alpha, \beta}}$ for all $\alpha, \beta \in \Xi$. So, there is a global section $\sigma_{\mathcal{E}}$ of $\mathcal{D}_{f}(\mathcal{E})$ such that $\operatorname{det} \lambda_{\alpha}=\left.\zeta_{\alpha} \sigma_{\mathcal{E}}\right|_{U_{\alpha}}$ for every $\alpha \in \Xi$.

We say that $\mathcal{D}_{f}(\mathcal{E})$ (equipped with $\zeta_{\alpha}$ for $\alpha \in \Xi(\mathcal{E})$ ) is the determinant of cohomology of $\mathcal{E}$ with respect to $f$. If $\chi(\mathcal{E} / S)=0$, we say that $\sigma_{\mathcal{E}}$ is the associated section. The zero-scheme of $\sigma_{\mathcal{E}}$ parameterizes the points $s \in S$ such that

$$
h^{0}(X(s), \mathcal{E}(s))=h^{1}(X(s), \mathcal{E}(s)) \neq 0 .
$$

(Another way of viewing $\sigma_{\mathcal{E}}$ is as a generator of the 0 -th Fitting ideal of $R^{1} f_{*} \mathcal{E}$.)

Proposition 44. The determinant of cohomology and its associated section satisfy the following properties. 
(1) (Base-change property) For every $S$-flat coherent sheaf $\mathcal{E}$ on $X$ and every Cartesian diagram

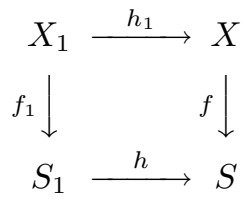

there is an induced isomorphism $\mathcal{D}_{f}(\mathcal{E}, h): h^{*} \mathcal{D}_{f}(\mathcal{E}) \cong \mathcal{D}_{f_{1}}\left(h_{1}^{*} \mathcal{E}\right)$. In addition, $\sigma_{h_{1}^{*} \mathcal{E}}=\mathcal{D}_{f}(\mathcal{E}, h) h^{*} \sigma_{\mathcal{E}}$ if $\chi(\mathcal{E} / S)=0$.

(2) (Functorial property) For every isomorphism $\phi: \mathcal{E} \cong \mathcal{F}$ of $S$-flat coherent sheaves on $X$, there is an induced isomorphism $\mathcal{D}_{f}(\phi): \mathcal{D}_{f}(\mathcal{E}) \cong \mathcal{D}_{f}(\mathcal{F})$. If $c \in H^{0}\left(S, \mathcal{O}_{S}\right)^{*}$, then $\mathcal{D}_{f}(c \phi)=c^{\chi(\mathcal{E} / S)} \mathcal{D}_{f}(\phi)$. If there is another isomorphism $\psi: \mathcal{F} \cong \mathcal{G}$, then $\mathcal{D}_{f}(\psi \phi)=\mathcal{D}_{f}(\psi) \mathcal{D}_{f}(\phi)$. If $\chi(\mathcal{E} / S)=0$, then $\sigma_{\mathcal{F}}=\mathcal{D}_{f}(\phi) \sigma_{\mathcal{E}}$. If there is a base-change diagram as in (1), then

$$
\mathcal{D}_{f}(\mathcal{F}, h) h^{*} \mathcal{D}_{f}(\phi)=\mathcal{D}_{f_{1}}\left(h_{1}^{*} \phi\right) \mathcal{D}_{f}(\mathcal{E}, h) .
$$

(3) (Projection property) For every $S$-flat coherent sheaf $\mathcal{E}$ on $X$ and every invertible sheaf $L$ on $S$, there is an induced isomorphism

$$
\mathcal{D}_{f}(\mathcal{E}, L): \mathcal{D}_{f}\left(\mathcal{E} \otimes f^{*} L\right) \cong \mathcal{D}_{f}(\mathcal{E}) \otimes L^{\otimes \chi(\mathcal{E} / S)} .
$$

If $M$ is another invertible sheaf on $S$, then

$$
\mathcal{D}_{f}(\mathcal{E}, L \otimes M)=\left(\mathcal{D}_{f}(\mathcal{E}, L) \otimes M^{\otimes \chi(\mathcal{E} / S)}\right) \mathcal{D}_{f}\left(\mathcal{E} \otimes f^{*} L, M\right) .
$$

If $\chi(\mathcal{E} / S)=0$, then $\sigma_{\mathcal{E}}=\mathcal{D}_{f}(\mathcal{E}, L) \sigma_{\mathcal{E} \otimes f^{*} L}$. If there is a base-change diagram as in (1), then

$$
\left(\mathcal{D}_{f}(\mathcal{E}, h) \otimes h^{*} L^{\otimes \chi(\mathcal{E} / S)}\right) h^{*} \mathcal{D}_{f}(\mathcal{E}, L)=\mathcal{D}_{f_{1}}\left(h_{1}^{*} \mathcal{E}, h^{*} L\right) \mathcal{D}_{f}\left(\mathcal{E} \otimes f^{*} L, h\right) .
$$

If there are isomorphisms $\phi: \mathcal{E} \cong \mathcal{F}$ and $\psi: L \cong M$, then

$$
\mathcal{D}_{f}(\mathcal{F}, M) \mathcal{D}_{f}\left(\phi \otimes f^{*} \psi\right)=\left(\mathcal{D}_{f}(\phi) \otimes \psi^{\otimes \chi(\mathcal{E} / S)}\right) \mathcal{D}_{f}(\mathcal{E}, L) .
$$

(4) (Additive property) For every short exact sequence

$$
\alpha: 0 \rightarrow \mathcal{E}_{1} \rightarrow \mathcal{E}_{2} \rightarrow \mathcal{E}_{3} \rightarrow 0
$$

of $S$-flat coherent sheaves on $X$, there is an induced isomorphism

$$
\mathcal{D}_{f}(\alpha): \mathcal{D}_{f}\left(\mathcal{E}_{2}\right) \cong \mathcal{D}_{f}\left(\mathcal{E}_{1}\right) \otimes \mathcal{D}_{f}\left(\mathcal{E}_{3}\right) \text {. }
$$

If $\chi\left(\mathcal{E}_{i} / S\right)=0$ for $i=1,2,3$, then $\sigma_{\mathcal{E}_{1}} \otimes \sigma_{\mathcal{E}_{3}}=\mathcal{D}_{f}(\alpha) \sigma_{\mathcal{E}_{2}}$. If there is a base-change diagram as in (1), then

$$
\left(\mathcal{D}_{f}\left(\mathcal{E}_{1}, h\right) \otimes \mathcal{D}_{f}\left(\mathcal{E}_{3}, h\right)\right) h^{*} \mathcal{D}_{f}(\alpha)=\mathcal{D}_{f_{1}}\left(h_{1}^{*} \alpha\right) \mathcal{D}_{f}\left(\mathcal{E}_{2}, h\right) .
$$

If there is an isomorphism of exact sequences

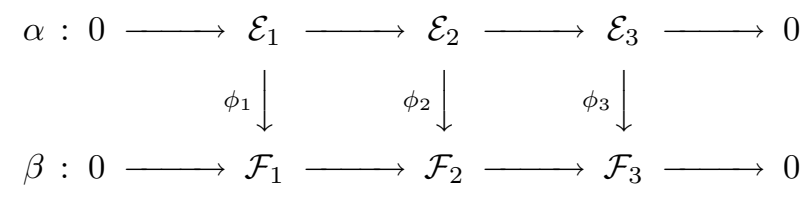

then

$$
\mathcal{D}_{f}(\beta) \mathcal{D}_{f}\left(\phi_{2}\right)=\left(\mathcal{D}_{f}\left(\phi_{1}\right) \otimes \mathcal{D}_{f}\left(\phi_{3}\right)\right) \mathcal{D}_{f}(\alpha) .
$$


If $L$ is an invertible sheaf on $S$, then

$$
\left(\mathcal{D}_{f}(\alpha) \otimes L^{\otimes \chi\left(\mathcal{E}_{2} / S\right)}\right) \mathcal{D}_{f}\left(\mathcal{E}_{2}, L\right)=\left(\mathcal{D}_{f}\left(\mathcal{E}_{1}, L\right) \otimes \mathcal{D}_{f}\left(\mathcal{E}_{3}, L\right)\right) \mathcal{D}_{f}\left(\alpha \otimes f^{*} L\right)
$$

Proof. We check (1). Since the formation of $R \cdot f_{*} \mathcal{E}$ commutes with base change, for every $\alpha \in \Xi$ we have $h^{*} \alpha:=\left(h^{-1}\left(U_{\alpha}\right), h^{*} F_{\alpha}, h^{*} p_{\alpha}\right) \in \Xi\left(h_{1}^{*} \mathcal{E}\right)$. In addition, $\lambda_{h^{*} \alpha}=h^{*} h_{\alpha}$ and $d_{h^{*} \alpha, h^{*} \beta}=\left.h\right|_{U_{h^{*} \alpha, h^{*} \beta}} ^{*} d_{\alpha, \beta}$ for all $\alpha, \beta \in \Xi$. It follows that there is an isomorphism $\mathcal{D}_{f}(\mathcal{E}, h): h^{*} \mathcal{D}_{f}(\mathcal{E}) \cong \mathcal{D}_{f_{1}}\left(h_{1}^{*} \mathcal{E}\right)$ such that

$$
\left.h\right|_{U_{h^{*} \alpha}} ^{*} \zeta_{\alpha}=\left.\zeta_{h^{*} \alpha} \mathcal{D}_{f}(\mathcal{E}, h)\right|_{U_{h^{*} \alpha}}
$$

for every $\alpha \in \Xi$. The last statement in (1) follows easily.

We check (2). If $\alpha \in \Xi$, then $\phi_{*} \alpha:=\left(U_{\alpha}, F_{\alpha}^{\cdot},\left.R f_{*} \phi\right|_{U_{\alpha}} p_{\alpha}\right) \in \Xi(\mathcal{F})$. In addition, $\lambda_{\phi_{*} \alpha}=\lambda_{\alpha}$ and $d_{\phi_{*} \alpha, \phi_{*} \beta}=d_{\alpha, \beta}$ for all $\alpha, \beta \in \Xi$. It follows that there is an isomorphism $\mathcal{D}_{f}(\phi): \mathcal{D}_{f}(\mathcal{E}) \cong \mathcal{D}_{f}(\mathcal{F})$ such that $\zeta_{\alpha}=\left.\zeta_{\phi_{*} \alpha} \mathcal{D}_{f}(\phi)\right|_{U_{\alpha}}$ for every $\alpha \in \Xi$. The remaining statements in (2) can be verified now.

We check (3). By the projection formula, $R \cdot f_{*} \mathcal{E} \otimes L=R f_{*}\left(\mathcal{E} \otimes f^{*} L\right)$. If $\alpha \in \Xi$ is such that $L$ is trivial on $U_{\alpha}$, then $\alpha \otimes L:=\left(U_{\alpha}, F_{\alpha}^{*} \otimes L, p_{\alpha} \otimes L\right) \in \Xi\left(\mathcal{E} \otimes f^{*} L\right)$. So, $\lambda_{\alpha \otimes L}=\lambda_{\alpha} \otimes L$ and $d_{\alpha \otimes L, \beta \otimes L}=d_{\alpha, \beta} \otimes L^{\otimes \chi(\mathcal{E} / S)}$ for all $\alpha, \beta \in \Xi$. It follows that there is an isomorphism $\mathcal{D}(\mathcal{E}, L): \mathcal{D}_{f}\left(\mathcal{E} \otimes f^{*} L\right) \cong \mathcal{D}_{f}(\mathcal{E}) \otimes L^{\otimes \chi}(\mathcal{E} / S)$ such that $\zeta_{\alpha \otimes L}=\left.\left(\left.\zeta_{\alpha} \otimes L\right|_{U_{\alpha}} ^{\otimes \chi(\mathcal{E} / S)}\right) \mathcal{D}_{f}(\mathcal{E}, L)\right|_{U_{\alpha}}$ for every $\alpha \in \Xi$ such that $L$ is trivial on $U_{\alpha}$. It is an exercise to check the remaining statements.

We check (4). Let $\phi_{i}: \mathcal{E}_{i} \rightarrow \mathcal{E}_{i+1}$ denote the maps given by $\alpha$, for $i=1,2$. Consider the collection $\Xi_{\alpha}$ formed by all quadruples $\beta:=\left(\beta_{1}, \beta_{2}, \beta_{3}, \Delta_{\beta}\right)$, where $\beta_{i} \in \Xi\left(\mathcal{E}_{i}\right)$ for $i=1,2,3$ are such that $U_{\beta_{1}}=U_{\beta_{2}}=U_{\beta_{3}}$, and

$$
\Delta_{\beta}: 0 \rightarrow F_{\beta_{1}}^{\cdot} \stackrel{h_{\beta, 1}}{\longrightarrow} F_{\beta_{2}}^{\cdot} \stackrel{h_{\beta, 2}}{\longrightarrow} F_{\beta_{3}} \rightarrow 0
$$

is a short exact sequence of complexes such that $p_{\beta_{i+1}} h_{\beta, i}=\left.R f_{*} \phi_{i}\right|_{U_{\beta_{1}}} p_{\beta_{i}}$ (up to homotopy) for $i=1,2$. Let $U_{\beta}:=U_{\beta_{1}}$. By the same argument used in Observation 43 , we can show that the $U_{\beta}$ cover $S$. If $\beta \in \Xi_{\alpha}$, by taking determinants in $\Delta_{\beta}$ we get a map

$$
\delta_{\beta}: \operatorname{det} F_{\beta_{2}} \rightarrow \operatorname{det} F_{\dot{\beta}_{1}} \otimes \operatorname{det} F_{\beta_{3}}
$$

For $\beta, \gamma \in \Xi_{\alpha}$ and $i=1,2,3$, let $U_{\beta, \gamma}:=U_{\beta} \cap U_{\gamma}$, and $F_{\beta_{i}, \gamma}, \lambda_{\beta_{i}, \gamma}$ and $h_{\beta, \gamma, i}$ denote the restrictions of $F_{\beta_{i}}, \lambda_{\beta_{i}}$ and $h_{\beta, i}$ to $U_{\beta, \gamma}$, respectively. In addition, let $g_{\beta_{i}, \gamma_{i}}: F_{\beta_{i}, \gamma}^{\cdot} \cong F_{\gamma_{i}, \beta}$ be quasi-isomorphisms such that $\left.p_{\beta_{i}}\right|_{U_{\beta, \gamma}}=\left.p_{\gamma_{i}}\right|_{U_{\beta, \gamma}} g_{\beta_{i}, \gamma_{i}}$ (up to homotopy). It follows that $g_{\beta_{i+1}, \gamma_{i+1}} h_{\beta, \gamma, i}=h_{\gamma, \beta, i} g_{\beta_{i}, \gamma_{i}}$ up to homotopy for $i=1,2$. In other words, for $i=1,2$ there is a map $\iota_{i}: F_{\beta_{i}, \gamma}^{1} \rightarrow F_{\gamma_{i+1}, \beta}^{0}$ such that

$$
\begin{aligned}
& h_{\gamma, \beta, i}^{0} g_{\beta_{i}, \gamma_{i}}^{0}-g_{\beta_{i+1}, \gamma_{i+1}}^{0} h_{\beta, \gamma, i}^{0}=\iota_{i} \lambda_{\beta_{i}, \gamma}, \\
& h_{\gamma, \beta, i}^{1} g_{\beta_{i}, \gamma_{i}}^{1}-g_{\beta_{i+1}, \gamma_{i+1}}^{1} h_{\beta, \gamma, i}^{1}=\lambda_{\gamma_{i+1}, \beta} \iota_{i} .
\end{aligned}
$$


For $\beta, \gamma \in \Xi_{\alpha}$ we can form the commutative diagram of exact sequences

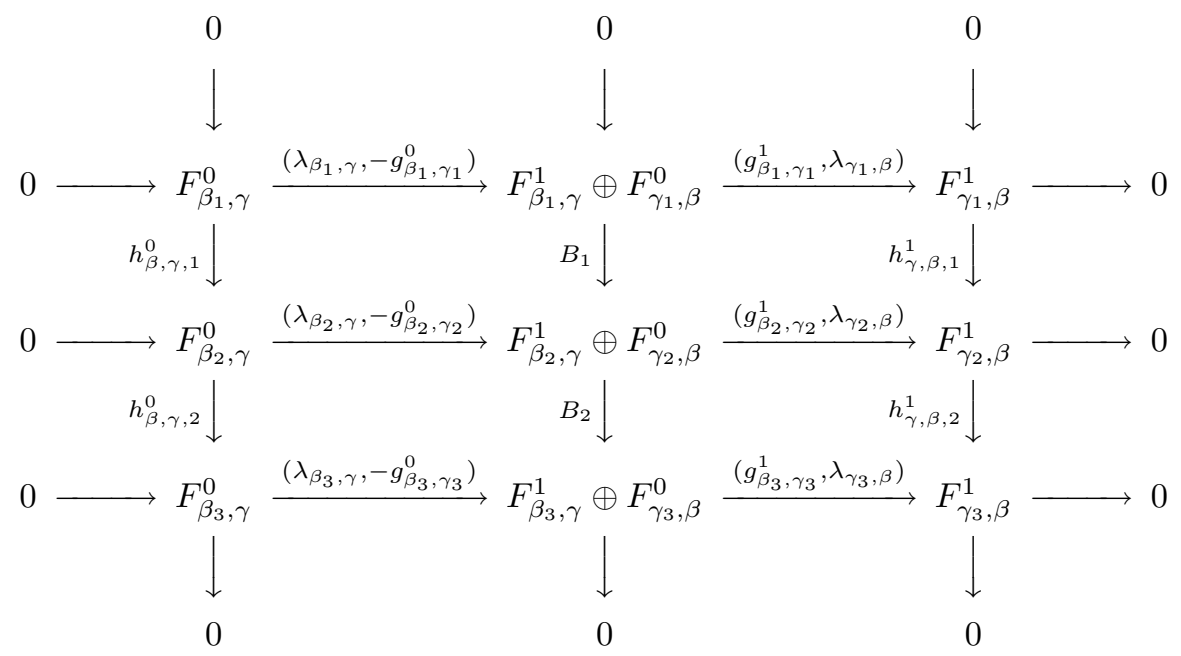

where

$$
B_{i}:=\left[\begin{array}{cc}
h_{\beta, \gamma, i}^{1} & 0 \\
\iota_{i} & h_{\gamma, \beta, i}^{0}
\end{array}\right]
$$

for $i=1,2$. Taking determinants, we get

$$
\left.\delta_{\gamma}\right|_{U_{\beta, \gamma}} d_{g_{\beta_{2}, \gamma_{2}}}=\left.(-1)^{\mathrm{rk}\left(F_{\gamma_{1}}^{0}\right) \operatorname{rk}\left(F_{\beta_{3}}^{1}\right)+\operatorname{rk}\left(F_{\gamma_{1}}^{1}\right) \operatorname{rk}\left(F_{\beta_{3}}^{0}\right)}\left(d_{g_{\beta_{1}, \gamma_{1}}} \otimes d_{g_{\beta_{3}, \gamma_{3}}}\right) \delta_{\beta}\right|_{U_{\beta, \gamma}} .
$$

Letting $d_{\beta}:=(-1)^{\mathrm{rk}\left(F_{\beta_{1}}^{0}\right)\left(\operatorname{rk}\left(F_{\beta_{3}}^{0}\right)+\operatorname{rk}\left(F_{\beta_{3}}^{1}\right)\right)} \delta_{\beta}$ for every $\beta \in \Xi_{\alpha}$, we have

$$
\left.d_{\gamma}\right|_{U_{\beta, \gamma}} d_{\beta_{2}, \gamma_{2}}=\left.\left(d_{\beta_{1}, \gamma_{1}} \otimes d_{\beta_{3}, \gamma_{3}}\right) d_{\beta}\right|_{U_{\beta, \gamma}} .
$$

It follows that there is an isomorphism $\mathcal{D}_{f}(\alpha): \mathcal{D}_{f}\left(\mathcal{E}_{2}\right) \cong \mathcal{D}_{f}\left(\mathcal{E}_{1}\right) \otimes \mathcal{D}_{f}\left(\mathcal{E}_{3}\right)$ such that $d_{\beta} \zeta_{\beta_{2}}=\left.\left(\zeta_{\beta_{1}} \otimes \zeta_{\beta_{3}}\right) \mathcal{D}_{f}(\alpha)\right|_{U_{\beta}}$ for every $\beta \in \Xi_{\alpha}$. We will leave it to the reader to verify the remaining statements.

For a more systematic development of the general theory of determinants and divisors, see [20]. It is also possible to adopt a more concrete approach to define the determinant of cohomology, like the one used in [4. Ch. IV, §3].

6.2. The theta line bundle and theta functions. Let $f: X \rightarrow S$ be a flat, projective map whose geometric fibers are connected curves. Fix an integer $d$, and let $\mathcal{E}$ be a vector bundle on $X$ of rank $r$ and $\operatorname{deg}(\mathcal{E} / S)=-r d$.

Construction 45 . Let $\mathcal{F}$ be a vector bundle on $X$ with rank $m$ and relative degree $-m d$ over $S$. Let $\Sigma \rightarrow J_{d}$ be an étale surjection such that $\Sigma$ is a scheme, and $X \times \Sigma$ admits a universal torsion-free, rank- 1 sheaf $\mathcal{I}_{1}$ over $\Sigma$. Let $p_{1}, p_{2}$ denote the projections from $X \times \Sigma$ onto the indicated factors. Let $\mathcal{I}_{2}$ be another universal sheaf on $X \times \Sigma / \Sigma$. Since $\mathcal{I}_{1}$ and $\mathcal{I}_{2}$ are simple, the sheaf $N_{1,2}:=p_{2 * \underline{H o m}_{X \times \Sigma}}\left(\mathcal{I}_{1}, \mathcal{I}_{2}\right)$ is invertible, and the canonical map $\nu_{1,2}: \mathcal{I}_{1} \otimes p_{2}^{*} N_{1,2} \rightarrow \mathcal{I}_{2}$ is an isomorphism by [3, Lemma 4.3, p. 79]. In addition, the formation of $N_{1,2}$ and $\nu_{1,2}$ commutes with base change. We obtain a canonical isomorphism

$h_{1,2}:=\mathcal{D}_{p_{2}}\left(\nu_{1,2} \otimes p_{1}^{*} \mathcal{F}\right) \mathcal{D}_{p_{2}}\left(\mathcal{I}_{1} \otimes p_{1}^{*} \mathcal{F}, N_{1,2}\right)^{-1}: \mathcal{D}_{p_{2}}\left(\mathcal{I}_{1} \otimes p_{1}^{*} \mathcal{F}\right) \cong \mathcal{D}_{p_{2}}\left(\mathcal{I}_{2} \otimes p_{1}^{*} \mathcal{F}\right)$ 
such that $\sigma_{\mathcal{I}_{2} \otimes p_{1}^{*} \mathcal{F}}=h_{1,2} \sigma_{\mathcal{I}_{1} \otimes p_{1}^{*} \mathcal{F}}$. The formation of $h_{1,2}$ commutes with base change. Let $\mathcal{I}_{3}$ be a third universal sheaf on $X \times \Sigma / \Sigma$. As before, let

$$
\begin{array}{ll}
N_{1,3}:=p_{2 *} \underline{\operatorname{Hom}}_{X \times \Sigma}\left(\mathcal{I}_{1}, \mathcal{I}_{3}\right), & \nu_{1,3}: \mathcal{I}_{1} \otimes p_{2}^{*} N_{1,3} \rightarrow \mathcal{I}_{3}, \\
N_{2,3}:=p_{2 *} \underline{\operatorname{Hom}}_{X \times \Sigma}\left(\mathcal{I}_{2}, \mathcal{I}_{3}\right), & \nu_{2,3}: \mathcal{I}_{2} \otimes p_{2}^{*} N_{2,3} \rightarrow \mathcal{I}_{3}
\end{array}
$$

where $\nu_{1,3}, \nu_{2,3}$ are canonical. Let $h: N_{1,2} \otimes N_{2,3} \rightarrow N_{1,3}$ be the natural map. Then, $h$ is an isomorphism and the diagram of maps

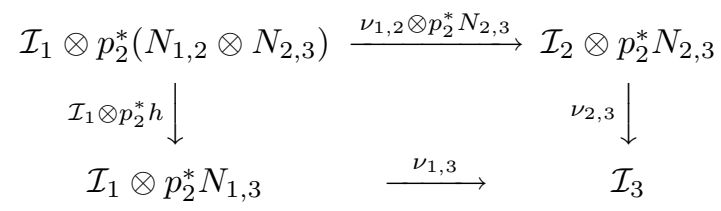

is commutative. Applying the functorial and projection properties of the determinant of cohomology to the above diagram, we get $h_{1,3}=h_{2,3} h_{1,2}$. We will apply the above construction to get sections of line bundles on $J_{d}$ below.

Let $\Xi$ denote the collection of all pairs $\alpha:=\left(\pi_{\alpha}, \mathcal{I}_{\alpha}\right)$, where $\pi_{\alpha}: \Sigma_{\alpha} \rightarrow J_{d}$ is an étale surjection from a scheme $\Sigma_{\alpha}$, and $\mathcal{I}_{\alpha}$ is a universal torsion-free, rank-1 sheaf on $X \times \Sigma_{\alpha} / \Sigma_{\alpha}$. Let $\mathcal{F}$ be a vector bundle on $X$ of $\operatorname{rank} m$ and $\operatorname{deg}(\mathcal{F} / S)=-m d$. Let $\mathcal{L}_{\mathcal{F}, \alpha}:=\mathcal{D}_{p_{\alpha, 2}}\left(\mathcal{I}_{\alpha} \otimes p_{\alpha, 1}^{*} \mathcal{F}\right)$ and $\theta_{\mathcal{F}, \alpha}:=\sigma_{\mathcal{I}_{\alpha} \otimes p_{\alpha, 1}^{*}} \mathcal{F}$, where $p_{\alpha, 1}, p_{\alpha, 2}$ are the projections from $X \times \Sigma_{\alpha}$ onto the indicated factors.

If $\alpha, \beta \in \Xi$, then $\left(1_{X}, \pi_{\alpha \cdot \beta, \alpha}\right)^{*} \mathcal{I}_{\alpha}$ and $\left(1_{X}, \pi_{\alpha \cdot \beta, \beta}\right)^{*} \mathcal{I}_{\beta}$ are universal torsion-free, rank-1 sheaves on $X \times \Sigma_{\alpha \cdot \beta}$ over the fibered product $\Sigma_{\alpha \cdot \beta}$ of $\pi_{\alpha}$ and $\pi_{\beta}$, where $\pi_{\alpha \cdot \beta, \alpha}$ and $\pi_{\alpha \cdot \beta, \beta}$ are the projections from $\Sigma_{\alpha \cdot \beta}$ onto $\Sigma_{\alpha}$ and $\Sigma_{\beta}$, respectively. By Construction 45 , we have an isomorphism $h_{\mathcal{F}, \alpha, \beta}: \pi_{\alpha \cdot \beta, \alpha}^{*} \mathcal{L}_{\mathcal{F}, \alpha} \cong \pi_{\alpha \cdot \beta, \beta}^{*} \mathcal{L}_{\mathcal{F}, \beta}$ such that $\pi_{\alpha \cdot \beta, \beta}^{*} \theta_{\mathcal{F}, \beta}=h_{\mathcal{F}, \alpha, \beta} \pi_{\alpha \cdot \beta, \alpha}^{*} \theta_{\mathcal{F}, \alpha}$. The formation of $h_{\mathcal{F}, \alpha, \beta}$ commutes with base change.

If $\alpha, \beta, \gamma \in \Xi$, then it follows from Construction 45 that

$$
q_{\alpha, \gamma}^{*} h_{\mathcal{F}, \alpha, \gamma}=q_{\beta, \gamma}^{*} h_{\mathcal{F}, \beta, \gamma} q_{\alpha, \beta}^{*} h_{\mathcal{F}, \alpha, \beta},
$$

where $q_{\alpha, \beta}, q_{\alpha, \gamma}, q_{\beta, \gamma}$ are the projections from $\Sigma_{\alpha} \times J_{d} \Sigma_{\beta} \times J_{d} \Sigma_{\gamma}$ onto the indicated factors. Since the cocycle condition is met, there are an invertible sheaf $\mathcal{L}_{\mathcal{F}}$ on $J_{d}$, a section $\theta_{\mathcal{F}} \in H^{0}\left(J_{d}, \mathcal{L}_{\mathcal{F}}\right)$ and isomorphisms $\zeta_{\mathcal{F}, \alpha}: \pi_{\alpha}^{*} \mathcal{L}_{\mathcal{F}} \cong \mathcal{L}_{\mathcal{F}, \alpha}$ for every $\alpha \in \Xi$ such that $\theta_{\mathcal{F}, \alpha}=\zeta_{\mathcal{F}, \alpha} \pi_{\alpha}^{*} \theta_{\mathcal{F}}$ and $\pi_{\alpha \cdot \beta, \beta}^{*} \zeta_{\mathcal{F}, \beta}=h_{\mathcal{F}, \alpha, \beta} \pi_{\alpha \cdot \beta, \alpha}^{*} \zeta_{\mathcal{F}, \alpha}$ for all $\alpha, \beta \in \Xi$. We say that $\mathcal{L}_{\mathcal{F}}$ is the theta line bundle on $J_{d}$ with respect to $\mathcal{F}$, and $\theta_{\mathcal{F}}$ is its associated theta function.

There is a natural way to produce sections of tensor powers of $\mathcal{L}_{\mathcal{E}}$, as we will describe after the next lemma.

Lemma 46. Let $\mathcal{F}_{1}$ and $\mathcal{F}_{2}$ be vector bundles on $X$ with the same rank, and let $\phi: \operatorname{det} \mathcal{F}_{1} \cong \operatorname{det} \mathcal{F}_{2}$ be an isomorphism. Then, there are an étale, surjective map $q: S^{\prime} \rightarrow S$, vector bundles $\mathcal{G}$ and $\mathcal{N}$ on $X \times S^{\prime}$, and extensions

$$
\alpha: 0 \rightarrow \mathcal{G} \rightarrow p_{1}^{*} \mathcal{F}_{1} \rightarrow \mathcal{N} \rightarrow 0 \quad \text { and } \quad \beta: 0 \rightarrow \mathcal{G} \rightarrow p_{1}^{*} \mathcal{F}_{2} \rightarrow \mathcal{N} \rightarrow 0
$$

whose induced isomorphisms

$$
\operatorname{det}(\alpha): p_{1}^{*} \operatorname{det} \mathcal{F}_{1} \rightarrow \operatorname{det} \mathcal{G} \otimes \operatorname{det} \mathcal{N} \quad \text { and } \quad \operatorname{det}(\beta): p_{1}^{*} \operatorname{det} \mathcal{F}_{2} \rightarrow \operatorname{det} \mathcal{G} \otimes \operatorname{det} \mathcal{N}
$$


satisfy $\operatorname{det}(\alpha)=\operatorname{det}(\beta) p_{1}^{*} \phi$, where $p_{1}, p_{2}$ are the projections from $X \times S^{\prime}$ onto the indicated factors. If $\mathcal{I}$ is a torsion-free, rank-1 sheaf on $X / S$, then the isomorphism

$$
\left(\mathcal{D}_{p_{2}}\left(p_{1}^{*} \mathcal{I} \otimes \beta\right)^{-1} \mathcal{D}_{p_{2}}\left(p_{1}^{*} \mathcal{I} \otimes \alpha\right)\right) \otimes\left(\mathcal{D}_{p_{2}}(\alpha)^{-1} \mathcal{D}_{p_{2}}(\beta)\right)
$$

descends to an isomorphism

$$
\tau:=\tau\left(\mathcal{I}, \mathcal{F}_{1}, \mathcal{F}_{2}, \phi\right): \mathcal{D}_{f}\left(\mathcal{I} \otimes \mathcal{F}_{1}\right) \otimes \mathcal{D}_{f}\left(\mathcal{F}_{2}\right) \rightarrow \mathcal{D}_{f}\left(\mathcal{I} \otimes \mathcal{F}_{2}\right) \otimes \mathcal{D}_{f}\left(\mathcal{F}_{1}\right) .
$$

The following properties are satisfied by $\tau$.

(1) (Independence) The map $\tau$ is independent of the choices of $q, \alpha$ and $\beta$. If $c \in H^{0}\left(S, \mathcal{O}_{S}\right)^{*}$, then $\tau\left(\mathcal{I}, \mathcal{F}_{1}, \mathcal{F}_{2}, c \phi\right)=c^{\chi\left(\mathcal{O}_{X} / S\right)-\chi(\mathcal{I} / S)} \tau$.

(2) (Base-change property) If

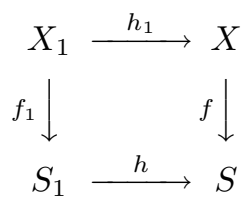

is a Cartesian diagram, then $h^{*} \tau=\tau\left(h_{1}^{*} \mathcal{I}, h_{1}^{*} \mathcal{F}_{1}, h_{1}^{*} \mathcal{F}_{2}, h_{1}^{*} \phi\right)$.

(3) (Functorial property) If $\psi: \mathcal{I} \rightarrow \mathcal{J}$ is an isomorphism, then

$$
\left(\mathcal{D}_{f}\left(\psi \otimes \mathcal{F}_{2}\right) \otimes \mathcal{D}_{f}\left(\mathcal{F}_{1}\right)\right) \tau=\tau\left(\mathcal{J}, \mathcal{F}_{1}, \mathcal{F}_{2}, \phi\right)\left(\mathcal{D}_{f}\left(\psi \otimes \mathcal{F}_{1}\right) \otimes \mathcal{D}_{f}\left(\mathcal{F}_{2}\right)\right) .
$$

(4) (Projection property) If $L$ is an invertible sheaf on $S$, then

$$
\begin{aligned}
& \begin{aligned}
\left(\mathcal{D}_{f}\left(\mathcal{I} \otimes \mathcal{F}_{2}, L\right) \otimes\right. & \left.\mathcal{D}_{f}\left(\mathcal{F}_{1}\right)\right) \tau\left(\mathcal{I} \otimes L, \mathcal{F}_{1}, \mathcal{F}_{2}, \phi\right) \\
& =\left(\tau \otimes L^{\otimes \chi}\right)\left(\mathcal{D}_{f}\left(\mathcal{I} \otimes \mathcal{F}_{1}, L\right) \otimes \mathcal{D}_{f}\left(\mathcal{F}_{2}\right)\right), \\
\text { where } \chi & :=\chi\left(\mathcal{I} \otimes \mathcal{F}_{1} / S\right)=\chi\left(\mathcal{I} \otimes \mathcal{F}_{2} / S\right) .
\end{aligned}
\end{aligned}
$$

Proof. The first statement was essentially proved in Lemma 17. As for the second statement, if $\mathcal{I}$ is torsion-free, rank- 1 on $X / S$, then $\mathcal{I}$ and $\mathcal{O}_{X}$ coincide generically in $K$-theory [12, p. 509]. Thus, the second statement and the property of independence follow from [12] Thm. I.1, p. 509]. The last properties follow from Proposition 44 and the expression for $q^{*} \tau$ given in the lemma.

Let $\mathcal{F}$ be a vector bundle on $X$ of rank $m r$ for some $m>0$. Assume there are isomorphisms $\phi: \operatorname{det} \mathcal{F} \cong(\operatorname{det} \mathcal{E})^{\otimes m}$ and $\psi: \mathcal{D}_{f}(\mathcal{E})^{\otimes m} \cong \mathcal{D}_{f}(\mathcal{F})$. Since the fibers of $X / S$ are geometrically reduced and connected, $\phi$ and $\psi$ are unique modulo $H^{0}\left(S, \mathcal{O}_{S}\right)^{*}$. Let $\alpha \in \Xi$ and $\rho_{\alpha}: \Sigma_{\alpha} \rightarrow S$ be the structure map. Let

$$
\tau_{\alpha}:=\tau\left(\mathcal{I}_{\alpha}, p_{\alpha, 1}^{*} \mathcal{F}, p_{\alpha, 1}^{*} \mathcal{E}^{\oplus m}, p_{\alpha, 1}^{*} \phi\right): \mathcal{L}_{\mathcal{F}, \alpha} \otimes \rho_{\alpha}^{*} \mathcal{D}_{f}(\mathcal{E})^{\otimes m} \cong \mathcal{L}_{\mathcal{E}, \alpha}^{\otimes m} \otimes \rho_{\alpha}^{*} \mathcal{D}_{f}(\mathcal{F}) .
$$

Let $\rho: J_{d} \rightarrow S$ be the structure map. It follows from Construction 45 and properties (1)-(4) of Lemma 46 that

$$
\left(h_{\mathcal{E}, \alpha, \beta}^{\otimes m} \otimes \rho_{\alpha \cdot \beta}^{*} \mathcal{D}_{f}(\mathcal{F})\right) \pi_{\alpha \cdot \beta, \alpha}^{*} \tau_{\alpha}=\pi_{\alpha \cdot \beta, \beta}^{*} \tau_{\beta}\left(h_{\mathcal{F}, \alpha, \beta} \otimes \rho_{\alpha \cdot \beta}^{*} \mathcal{D}_{f}(\mathcal{E})^{\otimes m}\right)
$$

for all $\alpha, \beta \in \Xi$, where $\rho_{\alpha \cdot \beta}: \Sigma_{\alpha \cdot \beta} \rightarrow S$ is the structure map. Thus, there is an isomorphism

$$
\tau: \mathcal{L}_{\mathcal{F}} \otimes \rho^{*} \mathcal{D}_{f}(\mathcal{E})^{\otimes m} \cong \mathcal{L}_{\mathcal{E}}^{\otimes m} \otimes \rho^{*} \mathcal{D}_{f}(\mathcal{F})
$$

such that $\left(\zeta_{\mathcal{E}, \alpha}^{\otimes m} \otimes 1\right) \pi_{\alpha}^{*} \tau=\tau_{\alpha}\left(\zeta_{\mathcal{F}, \alpha} \otimes 1\right)$ for every $\alpha \in \Xi$. Using $\psi$, we get an isomorphism $\mathcal{L}_{\mathcal{F}} \cong \mathcal{L}_{\mathcal{E}}^{\otimes m}$, by means of which we may regard $\theta_{\mathcal{F}}$ as a section of 
$\mathcal{L}_{\mathcal{E}}^{\otimes m}$. As such, $\theta_{\mathcal{F}}$ is well defined modulo our choices of $\phi$ and $\psi$, hence modulo $H^{0}\left(S, \mathcal{O}_{S}\right)^{*}$.

The theta functions allow us to produce separated realizations of pieces of $J_{d}$, as we will show in the next section.

\section{Separated Realizations}

7.1. By theta line bundles. Let $f: X \rightarrow S$ be a flat, projective map whose geometric fibers are connected curves. Let $d$ be an integer and $\Sigma \subseteq J_{d}$ an open subscheme with structure map $\pi: \Sigma \rightarrow S$ of finite type. Let $\mathcal{E}$ be a vector bundle on $X$ of $\operatorname{rank} r$ and $\operatorname{deg}(\mathcal{E} / S)=-r d$, and put $\mathcal{L}_{\mathcal{E} \mid \Sigma}:=\left.\mathcal{L}_{\mathcal{E}}\right|_{\Sigma}$. We say that $\mathcal{L}_{\mathcal{E} \mid \Sigma}$ is the theta line bundle on $\Sigma$.

Since $S$ is locally Noetherian and $\pi$ is of finite type, $\pi_{*} \mathcal{L}_{\mathcal{E} \mid \Sigma}^{\otimes m}$ is quasi-coherent for every $m \geq 0$. Let

$$
\Gamma_{\mathcal{E} \mid \Sigma}:=\bigoplus_{m \geq 0} \pi_{*} \mathcal{L}_{\mathcal{E} \mid \Sigma}^{\otimes m},
$$

and put $\tilde{\Sigma}_{\mathcal{E}}:=\operatorname{Proj}\left(\Gamma_{\mathcal{E} \mid \Sigma}\right)$. Since $\Gamma_{\mathcal{E} \mid \Sigma}$ is quasi-coherent, $\tilde{\Sigma}_{\mathcal{E}}$ is separated over $S$. Let $\psi: \Sigma \rightarrow \tilde{\Sigma}_{\mathcal{E}}$ denote the canonical rational map associated with $\Gamma_{\mathcal{E} \mid \Sigma}$. The scheme $\tilde{\Sigma}_{\mathcal{E}}$ can be regarded as a separated realization of $\Sigma$.

Example 47. We go back to Example 39, and use the notation therein. We showed there that $J_{M}=J_{E}^{p}$ for any non-singular point $p \in X$, where $E:=M^{*} \otimes \mathcal{O}_{X}(p)$. Even though the scheme $J_{M}$ does not depend on the choice of $p$, the polarization $E$ does. Therefore, we can expect the rational map $\psi: J_{M} \rightarrow \tilde{J}_{M, E}$ to depend on $p$. In fact, let $L_{E}$ denote the theta line bundle on $J_{M}$ with respect to $E$. It follows from the base-change property of the determinant of cohomology that $\alpha_{M}^{*}\left(L_{E}\right) \cong \mathcal{O}_{X}(p)$. So, under the identification between $X$ and $J_{M}$ given by $\alpha_{M}$, we see that $\tilde{J}_{M, E} \cong \tilde{X}_{p}$, where $X_{p}$ is the irreducible component of $X$ containing $p$, and $\tilde{X}_{p}$ is the quotient of $X_{p}$ obtained by identifying all points lying in the same connected component of $X_{p}^{c}$, for every such connected component.

Theorem 48. Let $\Sigma \subseteq J_{\mathcal{E}}^{s s}$ be an open subspace. Let $\psi: \Sigma \rightarrow \tilde{\Sigma}_{\mathcal{E}}$ denote the natural rational map. Then, the following statements hold.

(1) The map $\psi$ is defined on $\Sigma$ and is scheme-theoretically dominant.

(2) Every fiber of $\psi(s)$ is contained in a JH-equivalence class of $\Sigma(s)$ for every $s \in S$.

(3) If $\Sigma$ contains $J_{\mathcal{E}}^{s}$ and is universally closed over $S$, then the restriction $\left.\psi\right|_{J_{\mathcal{E}}^{s}}$ is an open embedding.

Proof. We show (1) first. Since $\pi: \Sigma \rightarrow S$ is of finite type and $S$ is locally Noetherian, the formation of $\Gamma_{\mathcal{E} \mid \Sigma}$ commutes with flat base change. By Lemma 36, we may assume $\Sigma$ is a scheme. Let $s \in S$ and $t \in \Sigma(s)$. There are a finite and separable field extension $k^{\prime} \supseteq k(t)$ and a semi-stable sheaf $I$ on $X\left(k^{\prime}\right)$ with respect to $\mathcal{E}\left(k^{\prime}\right)$ representing $t$. By Theorem 19, up to replacing $S$ by an étale neighborhood of $s$ and $k^{\prime}$ by a finite and separable field extension, we may assume that there is a vector bundle $\mathcal{F}$ on $X$ such that $\operatorname{det} \mathcal{F} \cong(\operatorname{det} \mathcal{E})^{\otimes 2}$ and

$$
h^{0}\left(X\left(k^{\prime}\right), I \otimes \mathcal{F}\left(k^{\prime}\right)\right)=h^{1}\left(X\left(k^{\prime}\right), I \otimes \mathcal{F}\left(k^{\prime}\right)\right)=0 .
$$


By Lemma 46, we may also assume $\mathcal{D}_{f}(\mathcal{E})^{\otimes 2} \cong \mathcal{D}_{f}(\mathcal{F})$. Let $\theta_{\mathcal{F}} \in H^{0}\left(J_{d}, \mathcal{L}_{\mathcal{E}}^{\otimes 2}\right)$ denote the corresponding theta function. It follows from $(48.1)$ that $\theta_{\mathcal{F}}(t) \neq 0$. Thus, $\psi$ is defined at $t$. It is clear that $\psi$ is scheme-theoretically dominant.

We prove (2). As before, we may assume $\Sigma$ is a scheme. Let $s \in S$. Since the formation of $\Gamma_{\mathcal{E}, \Sigma}$ commutes with localization, we may assume $S:=\operatorname{Spec}\left(\mathcal{O}_{S, s}\right)$. Let $t_{1}, t_{2} \in \Sigma(s)$. For $i=1,2$ there are a finite and separable field extension $k_{i} \supseteq k\left(t_{i}\right)$ and a semi-stable sheaf $I_{i}$ on $X\left(k_{i}\right)$ with respect to $\mathcal{E}\left(k_{i}\right)$ representing $t_{i}$. Let $k_{3} \supseteq k(s)$ be an algebraically closed field extension containing $k_{1}$ and $k_{2}$. Assume $\operatorname{Gr}\left(I_{1}\left(k_{3}\right)\right) \neq \operatorname{Gr}\left(I_{2}\left(k_{3}\right)\right)$. By [10, Lemma 10], there are an integer $m$ and a vector bundle $F$ on $X\left(k_{3}\right)$ of rank $m r$ and $\operatorname{det} F \cong\left(\operatorname{det} \mathcal{E}\left(k_{3}\right)\right)^{\otimes m}$ such that

$$
\begin{aligned}
& h^{0}\left(X\left(k_{3}\right), I_{1}\left(k_{3}\right) \otimes F\right)=h^{1}\left(X\left(k_{3}\right), I_{2}\left(k_{3}\right) \otimes F\right) \neq 0, \\
& h^{0}\left(X\left(k_{3}\right), I_{2}\left(k_{3}\right) \otimes F\right)=h^{1}\left(X\left(k_{3}\right), I_{2}\left(k_{3}\right) \otimes F\right)=0 .
\end{aligned}
$$

By [14, III-1, 10.3.1, p. 20], there is a local, Noetherian, flat $\mathcal{O}_{S, s}$-algebra $A$ with residue field $k_{3}$. Replacing $\mathcal{O}_{S, s}$ by $A$, we may assume $k_{3}=k(s)$. By Lemma 17 , up to replacing $S$ by an étale neighborhood of $s$, we may assume there is a vector bundle $\mathcal{F}$ on $X$ such that $\operatorname{det} \mathcal{F} \cong(\operatorname{det} \mathcal{E})^{\otimes m}$ and $\mathcal{F}(s) \cong F$. By Lemma 46 , we may also assume $\mathcal{D}_{f}(\mathcal{E})^{\otimes m} \cong \mathcal{D}_{f}(\mathcal{F})$. Let $\theta_{\mathcal{F}} \in H^{0}\left(J_{d}, \mathcal{L}_{\mathcal{E}}^{\otimes m}\right)$ denote the corresponding theta function. It follows from (48.2) that $\theta_{\mathcal{F}}\left(t_{1}\right)=0$ but $\theta_{\mathcal{F}}\left(t_{2}\right) \neq 0$. Thus, $\psi\left(t_{1}\right) \neq \psi\left(t_{2}\right)$.

We prove (3). As before, we may assume $\Sigma$ is a scheme. In addition, we may assume $X \times \Sigma$ admits a universal torsion-free, rank-1 sheaf $\mathbf{I}$ over $\Sigma$. Since $\Sigma$ is universally closed over $S$ and $\psi$ is dominant, $\psi$ is surjective and closed. It follows from (2) that $\psi\left(J_{\mathcal{E}}^{s}\right)$ and $\psi\left(\Sigma \backslash J_{\mathcal{E}}^{s}\right)$ are disjoint. Hence, $\tilde{J}_{\mathcal{E}}^{s}:=\psi\left(J_{\mathcal{E}}^{s}\right)$ is open in $\tilde{\Sigma}_{\mathcal{E}}$ and $J_{\mathcal{E}}^{s}=\psi^{-1}\left(\tilde{J}_{\mathcal{E}}^{s}\right)$. In addition, it follows from (2) that the restriction

$$
\phi:=\left.\psi\right|_{J_{\mathcal{E}}^{s}}: J_{\mathcal{E}}^{s} \rightarrow \tilde{J}_{\mathcal{E}}^{s}
$$

is bijective. Since $\pi: \Sigma \rightarrow S$ is universally closed, so is $\phi$. Since $J_{\mathcal{E}}^{s}$ is separated over $S$, it follows that $\phi$ is proper. Since $\phi$ is bijective and proper, $\phi$ is finite. We need only show that $\phi$ separates tangent vectors.

Let $s \in S$ and $t \in J_{\mathcal{E}}^{S}(s)$. Let $R:=k(t)[\epsilon] /\left(\epsilon^{2}\right)$ and $B:=\operatorname{Spec}(R)$. Let $b \in B$. Let $\nu: B \rightarrow J_{\mathcal{E}}^{s}$ be a non-constant map with $\nu(b)=t$ and $\pi \nu(B)=s$. We claim that $\phi \nu$ is non-constant as well. First, we show the claim is local in the flat topology. Indeed, if $S^{\prime} \rightarrow S$ is a flat map and $s^{\prime} \in S^{\prime}$ lies above $s$, let $t^{\prime} \in J_{\mathcal{E}}^{s}\left(s^{\prime}\right)$ lie above $t$, and put $R^{\prime}:=k\left(t^{\prime}\right)[\epsilon] /\left(\epsilon^{2}\right)$ and $B^{\prime}:=\operatorname{Spec}\left(R^{\prime}\right)$. Let $b^{\prime} \in B^{\prime}$. Since $\nu(B)=s$, the map $\nu$ lifts over $S^{\prime}$ to a map $\nu^{\prime}: B^{\prime} \rightarrow J_{\mathcal{E}}^{s} \times S^{\prime}$ with $\left(\pi \times 1_{S^{\prime}}\right) \nu^{\prime}(B)=s^{\prime}$. Since $\nu$ is not constant, neither is $\nu^{\prime}$. Since $\left(\pi \times 1_{S^{\prime}}\right) \nu^{\prime}(B)=s^{\prime}$, if we show that $\left(\phi \times 1_{S^{\prime}}\right) \nu^{\prime}$ is non-constant, then $\phi \nu$ is non-constant.

As shown above, we may assume $S:=\operatorname{Spec}\left(\mathcal{O}_{S, s}\right)$. Up to replacing $\mathcal{O}_{S, s}$ by a local, Noetherian, flat $\mathcal{O}_{S, s^{-}}$-algebra, we may also assume $k(t)=k(s)$, and $k(s)$ is algebraically closed [14, III-1, 10.3.1, p. 20]. Let $\mathcal{I}$ be the pullback of $\mathbf{I}$ to $X \times B$. Since $\nu$ is non-trivial, and is represented by $\mathcal{I}$, it follows that $\mathcal{I}$ is a non-trivial deformation of $\mathcal{I}(b)$. By [10, Lemma 12], there are an integer $m$ and a vector bundle $F$ on $X(s)$ of rank $m r$ and $\operatorname{det} F \cong(\operatorname{det} \mathcal{E}(s))^{\otimes m}$ such that the zero scheme of $\sigma_{\mathcal{I} \otimes\left(F \otimes \mathcal{O}_{B}\right)}$ is (reduced and) equal to $b$. By Lemma 17, up to replacing $S$ by an étale neighborhood of $s$, we may assume there is a vector bundle $\mathcal{F}$ on $X$ such that $\operatorname{det} \mathcal{F} \cong(\operatorname{det} \mathcal{E})^{\otimes m}$ and $\mathcal{F}(s) \cong F$. By Lemma 46 , up to replacing $S$ by an étale covering, we may also assume $\mathcal{D}_{f}(\mathcal{E})^{\otimes m} \cong \mathcal{D}_{f}(\mathcal{F})$. Let $\theta_{\mathcal{F}} \in H^{0}\left(J_{d}, \mathcal{L}_{\mathcal{E}}^{\otimes m}\right)$ denote 
the corresponding theta function. By the base-change property of the determinant of cohomology, the zero scheme of $\nu^{*} \theta_{\mathcal{F}}$ is equal to that of $\sigma_{\mathcal{I} \otimes\left(F \otimes \mathcal{O}_{B}\right)}$. So, $\phi \nu$ is non-constant, and hence $\phi$ separates tangent vectors.

Remark 49. Let $e(X / S)$ be the maximum number of generators at all points of $X(s)$ of all torsion-free, rank-1 sheaves on $X(s)$ for all $s \in S$. If the fibers of $X / S$ have only double points for singularities, then $e(X / S)=2$ (see [11]). In any case, we can show that $e(X / S) \leq g+1$, where $g$ is the (locally constant) arithmetic genus of the curves in the family $X / S$ (cf. [9, Rmk. 8, p. 189]). For every integer $m \geq 0$, let $\Gamma_{\mathcal{E} \mid \Sigma}(m) \subseteq \Gamma_{\mathcal{E} \mid \Sigma}$ be the subsheaf of graded $\mathcal{O}_{S}$-subalgebras generated by the homogeneous pieces $\pi_{*} \mathcal{L}_{\mathcal{E} \mid \Sigma}^{\otimes i}$ with $0 \leq i \leq m$. Let $\tilde{\Sigma}_{\mathcal{E}}(m):=\operatorname{Proj}\left(\Gamma_{\mathcal{E} \mid \Sigma}(m)\right)$ and $\psi_{m}: \Sigma \rightarrow \tilde{\Sigma}_{\mathcal{E}}(m)$ denote the associated rational map. We can show that the properties of Theorem 48 hold already for $\psi_{m}$ if $m>\max (e(X / S), 2)$ (cf. [9. Thm. 7, p. 187]). We omit the proof as it would require a certain amount of preliminary work to establish effective separation properties for theta functions. In addition, it is not known whether $\max (e(X / S), 2)$ is a sharp bound or not.

Corollary 50. $J_{\mathcal{E}}^{s}$ is a scheme.

Proof. Apply statement (3) of Theorem 48 to $\Sigma:=J_{\mathcal{E}}^{s s}$.

Proof of Theorem B. By Lemma 35, there are polarizations $\mathcal{E}_{j}$ on $X / S$ such that $J=\bigcup_{j} J_{\mathcal{E}_{j}}^{s}$. By Corollary 50 , the space $J_{\mathcal{E}_{j}}^{s}$ is a scheme for every $j$. So, $J$ is a scheme.

Remark 51. I conjecture that $J$ is a scheme if the irreducible components of every fiber of $f$ are geometrically integral. I can prove my conjecture when $S$ is (the spectrum of) a field, by showing that there are enough polarizations on $X$. In this case, the argument for constructing polarizations is just an adaptation of the one used in the proof of Lemma 35. If one could prove the conjecture in general, then one would obtain a true generalization of Mumford's result [6, p. 210].

Corollary 52. There is an étale surjection $S^{\prime} \rightarrow S$ such that $J \times{ }_{S} S^{\prime}$ is a scheme.

Proof. The proof is immediate from Lemma 18 and Theorem B.

Remark 53. If $\pi: \Sigma \rightarrow S$ is proper, then $\pi_{*} \mathcal{L}_{\mathcal{E} \mid \Sigma}^{\otimes m}$ is coherent for all $m \geq 0$. By Theorem A, this is the case when $\Sigma=J_{\mathcal{E}}^{\sigma}$. It follows that $\pi_{*} \mathcal{L}_{\mathcal{E} \mid \Sigma}^{\otimes m}$ is coherent as well for every $m \geq 0$ when $\Sigma=J_{\mathcal{E}}^{q s}$. In fact, by Lemma 18, up to replacing $S$ by an étale covering, we may assume there are sections $\sigma_{1}, \ldots, \sigma_{n}: S \rightarrow X$ of $f$ through the $S$-smooth locus of $X$ such that $J_{\mathcal{E}}^{q s}=J_{\mathcal{E}}^{\sigma_{1}} \cup \cdots \cup J_{\mathcal{E}}^{\sigma_{n}}$. For $i=1, \ldots, n$, let $\pi_{i}: J_{\mathcal{E}}^{\sigma_{i}} \rightarrow S$ denote the structure map. Since

$$
\pi_{*} \mathcal{L}_{\mathcal{E} \mid J_{\mathcal{E}}^{q s}}^{\otimes m} \subseteq \bigoplus_{i=1}^{n} \pi_{i *} \mathcal{L}_{\mathcal{E} \mid J_{\mathcal{E}}^{\sigma_{i}}}^{\otimes m}
$$

and the $\pi_{i *} \mathcal{L}_{\mathcal{E} \mid J_{\mathcal{E}}^{\sigma_{i}}}^{\otimes m}$ are coherent, so is $\pi_{*} \mathcal{L}_{\mathcal{E} \mid J_{\mathcal{E}}^{q s}}^{\otimes m}$.

In the proof of Theorem 48 we used only theta functions. We shall consider realizations defined only by theta functions in the next subsection. 
7.2. By theta functions. Let $f: X \rightarrow S$ be a flat, projective map whose geometric fibers are connected curves. Let $d$ be an integer and $\Sigma \subseteq J_{d}$ an open subscheme with structure map $\pi: \Sigma \rightarrow S$ of finite type. Let $\mathcal{E}$ be a vector bundle on $X$ of rank $r$ and $\operatorname{deg}(\mathcal{E} / S)=-r d$.

For each integer $m \geq 0$ and each open subscheme $U \subseteq S$, let $\tilde{A}_{\mathcal{E} \mid \Sigma}^{m}(U)$ be the $H^{0}\left(U, \mathcal{O}_{S}\right)$-submodule of $H^{0}\left(\Sigma \times U, \mathcal{L}_{\mathcal{E} \mid \Sigma}^{\otimes m}\right)$ generated by the restrictions of theta functions $\theta_{\mathcal{F}} \in H^{0}\left(J_{d} \times U, \mathcal{L}_{\mathcal{E}}^{\otimes m}\right)$ associated to vector bundles $\mathcal{F}$ on $X \times U$ of rank $m r$ and isomorphisms det $\mathcal{F} \cong(\operatorname{det} \mathcal{E})^{\otimes m} \otimes \mathcal{O}_{U}$ and $\left.\mathcal{D}_{f}(\mathcal{E})^{\otimes m}\right|_{U} \cong \mathcal{D}_{f_{U}}(\mathcal{F})$, where $f_{U}: X \times U \rightarrow U$ is the projection. Since the above isomorphisms are unique modulo $H^{0}\left(U, \mathcal{O}_{S}\right)^{*}$, the submodule $\tilde{A}_{\mathcal{E} \mid \Sigma}^{m}(U)$ is well-defined (see Subsection 6.2). By the base-change property of the determinant of cohomology, $\tilde{A}_{\mathcal{E} \mid \Sigma}^{m}$ is a presheaf. Let $A_{\mathcal{E} \mid \Sigma}^{m}$ denote the associated sheaf in the étale topology. We say that $A_{\mathcal{E} \mid \Sigma}^{m}$ is the sheaf of theta functions of degree $m$ associated to $\mathcal{E}$ and $\Sigma$.

The proof of the following lemma was communicated to me by M. Homma [16].

Lemma 54. Let $A$ be a Noetherian ring, $h$ and $t$ positive integers. There are a faithfully flat, étale $A$-algebra $B$, a $B$-basis $p_{1}, \ldots, p_{u}$ of the vector space of degree$h$ homogeneous polynomials of $B\left[Y_{1}, \ldots, Y_{t}\right]$, and linear $q_{1}, \ldots, q_{u} \in B\left[Y_{1}, \ldots, Y_{t}\right]$, such that, for every $t$-uple $\left(M_{1}, \ldots, M_{t}\right)$ of square matrices of size $h$ and entries in a B-algebra $C$, and every $\left(y_{1}, \ldots, y_{t}\right) \in C^{\oplus t}$,

$$
\operatorname{det}\left(y_{1} M_{1}+\cdots+y_{t} M_{t}\right)=\sum_{i=1}^{u} p_{i}\left(y_{1}, \ldots, y_{t}\right) \operatorname{det} q_{i}\left(M_{1}, \ldots, M_{t}\right) .
$$

If $A$ contains an infinite field, we may take $B=A$.

Proof. Let $C$ be an $A$-algebra, and $M_{1}, \ldots, M_{t}$ square matrices of size $h$ and entries in $C$. For $i=1, \ldots, t$ and $j=1, \ldots, h$ let $v_{i, j} \in C^{\oplus h}$ be the $j$-th row vector of $M_{i}$. For every $h$-uple $\underline{d}:=\left(d_{1}, \ldots, d_{h}\right)$ of positive integers $d_{j}$ with $1 \leq d_{j} \leq t$, let $M_{\underline{d}}$ be the matrix whose $j$-th row is $v_{d_{j}, j}$ for $j=1, \ldots, h$. For each $t$-uple $\underline{e}:=\left(e_{1}, \ldots, e_{t}\right)$ of non-negative integers with $e_{1}+\cdots+e_{t}=h$, let $g_{\underline{e}}:=\sum \operatorname{det} M_{\underline{d}}$, with the sum ranging over all $h$-uples $\underline{d}$ where $i$ appears exactly $e_{i}$ times as a component for $i=1, \ldots, t$. Then,

$$
\operatorname{det}\left(y_{1} M_{1}+\cdots+y_{t} M_{t}\right)=\sum_{\underline{e}} g_{\underline{e}} y_{1}^{e_{1}} \ldots y_{t}^{e_{t}}
$$

for every $t$-uple $\left(y_{1}, \ldots, y_{t}\right) \in C^{\oplus t}$.

Consider the Veronese embedding $\nu: \mathbf{P}_{A}^{t-1} \rightarrow \mathbf{P}_{A}^{u-1}$, sending $\left(y_{1}: y_{2}: \cdots: y_{t}\right)$ to $\left(\ldots: y_{1}^{e_{1}} y_{2}^{e_{2}} \cdots y_{t}^{e_{t}}: \ldots\right)$. Since the image of $\nu$ has non-degenerate fibers over $\operatorname{Spec}(A)$, applying [14, IV-4, 17.16.3] repeatedly we get a faithfully flat, étale $A$ algebra $B$ and $B$-points $x_{i}:=\left(b_{i, 1}: \ldots: b_{i, t}\right) \in \mathbf{P}_{B}^{t-1}$ for $i=1, \ldots, u$ such that $\nu\left(x_{1}\right), \ldots, \nu\left(x_{u}\right)$ span $\mathbf{P}_{B}^{u-1}$. Equivalently, the matrix $W:=\left(w_{i, \underline{e}}\right)$ is invertible, where $w_{i, \underline{e}}:=b_{i, 1}^{e_{1}} \cdots b_{i, t}^{e_{t}}$ for $1 \leq i \leq u$ and all $t$-uples $\underline{e}:=\left(e_{1}, \ldots, e_{t}\right)$ of nonnegative integers with $e_{1}+\cdots+e_{t}=h$. Let $q_{i}:=b_{i, 1} Y_{1}+\cdots+b_{i, t} Y_{t}$ for $i=1, \ldots, t$. Since $W$ is invertible, it follows from (54.1) that the $g_{\underline{e}}$ are expressible as $B$-linear combinations of the determinants of the matrices $q_{i}\left(M_{1}, \ldots, M_{t}\right)$. Just replace these expressions for the $g_{\underline{e}}$ in (54.1), and rearrange the sum.

If $A$ contains an infinite field $k$, then we may find enough $k$-points in the image of the Veronese embedding to span $\mathbf{P}_{k}^{u-1}$. So, we may take $B=A$. 
Construction 55. Assume there is a universal torsion-free, rank-1 sheaf $\mathcal{I}$ on $X \times \Sigma$ over $\Sigma$. Assume there are an integer $m$, vector bundles $\mathcal{G}_{1}$ and $\mathcal{G}_{2}$ on $X$, and an extension

$$
\alpha: 0 \rightarrow \mathcal{G}_{2} \rightarrow \mathcal{E}^{\oplus m} \rightarrow \mathcal{G}_{1} \rightarrow 0
$$

such that

$$
h^{0}\left(X(s), \mathcal{I}(s) \otimes \mathcal{G}_{2}(s)\right)=h^{1}\left(X(s), \mathcal{I}(s) \otimes \mathcal{G}_{1}(s)\right)=0
$$

for every $s \in \Sigma$. Let $p_{1}, p_{2}$ denote the projections from $X \times \Sigma$ onto the indicated factors. For every extension

$$
\beta: 0 \rightarrow \mathcal{G}_{2} \rightarrow \mathcal{F} \rightarrow \mathcal{G}_{1} \rightarrow 0
$$

consider the complex

$$
C_{\beta}^{\cdot}: 0 \rightarrow p_{2 *}\left(\mathcal{I} \otimes p_{1}^{*} \mathcal{G}_{1}\right) \stackrel{\lambda_{\beta}}{\longrightarrow} R^{1} p_{2 *}\left(\mathcal{I} \otimes p_{1}^{*} \mathcal{G}_{2}\right) \rightarrow 0
$$

obtained by tensoring $p_{1}^{*} \beta$ with $\mathcal{I}$, and taking direct images. Because of (55.1), the sheaves in $C_{\beta}$ are locally free, say of rank $h$. Moreover,

$$
\begin{aligned}
& \mathcal{D}_{p_{2}}\left(\mathcal{I} \otimes p_{1}^{*} \mathcal{G}_{1}\right)=\operatorname{det} p_{2 *}\left(\mathcal{I} \otimes p_{1}^{*} \mathcal{G}_{1}\right), \\
& \mathcal{D}_{p_{2}}\left(\mathcal{I} \otimes p_{1}^{*} \mathcal{G}_{2}\right)=\operatorname{det} R^{1} p_{2 *}\left(\mathcal{I} \otimes p_{1}^{*} \mathcal{G}_{2}\right)
\end{aligned}
$$

and the complex $C_{\beta}$ is quasi-isomorphic to $R^{*} p_{2 *}\left(\mathcal{I} \otimes p_{1}^{*} \mathcal{F}\right)$. So, there is an isomorphism $\zeta_{\beta}: \mathcal{D}_{p_{2}}\left(\mathcal{I} \otimes p_{1}^{*} \mathcal{F}\right) \cong \operatorname{det} C_{\beta}$ such that $\sigma_{\beta}=\zeta_{\beta} \sigma_{\mathcal{I} \otimes p_{1}^{*} \mathcal{F}}$, where

$$
\sigma_{\beta}:=\operatorname{det}\left(\lambda_{\beta}\right) \otimes \operatorname{det}\left(p_{2 *}\left(\mathcal{I} \otimes p_{1}^{*} \mathcal{G}_{1}\right)\right)^{-1} \text {. }
$$

Under the identifications (55.2), we have $\zeta_{\beta}=\mathcal{D}_{p_{2}}(\mathcal{I} \otimes \beta)$. Let $\theta_{\mathcal{F} \mid \Sigma}$ be the restriction to $\Sigma$ of the section $\theta_{\mathcal{F}} \in H^{0}\left(J_{d}, \mathcal{L}_{\mathcal{E}}^{\otimes m}\right)$ constructed out of $\mathcal{F}$ and the isomorphisms $\phi_{\beta}:=\operatorname{det}(\alpha)^{-1} \operatorname{det}(\beta)$ and $\psi_{\beta}:=\mathcal{D}_{f}(\beta)^{-1} \mathcal{D}_{f}(\alpha)$ (see Subsection 6.2). By Lemma 46 ,

$$
\theta_{\mathcal{F} \mid \Sigma}=\zeta_{\alpha}^{-1} \zeta_{\beta} \sigma_{\mathcal{I} \otimes p_{1}^{*} \mathcal{F}}=\zeta_{\alpha}^{-1} \sigma_{\beta}
$$

Assume $S:=\operatorname{Spec}(A)$. For $i=1, \ldots, t$, let

$$
\beta_{i}: 0 \rightarrow \mathcal{G}_{2} \rightarrow \mathcal{F}_{i} \rightarrow \mathcal{G}_{1} \rightarrow 0
$$

be an extension. Let $B$ and $p_{1}, \ldots, p_{u}, q_{1}, \ldots, q_{u} \in B\left[Y_{1}, \ldots, Y_{t}\right]$ be as in Lemma 54 . Let $C$ be a Noetherian $B$-algebra, and set $T:=\operatorname{Spec}(B)$ and $U:=\operatorname{Spec}(C)$. For every $c:=\left(c_{1}, \ldots, c_{t}\right) \in C^{\oplus t}$, let

$$
\beta_{c}: 0 \rightarrow \mathcal{G}_{2} \otimes \mathcal{O}_{U} \rightarrow \mathcal{F}_{c} \rightarrow \mathcal{G}_{1} \otimes \mathcal{O}_{U} \rightarrow 0
$$

be the $c$-weighted sum of the $\beta_{i}$, that is, $\beta_{c}:=c_{1} \beta_{1}+\cdots+c_{t} \beta_{t}$. By linearity, $\lambda_{\beta_{c}}=c_{1} \lambda_{\beta_{1}}+\cdots+c_{t} \lambda_{\beta_{t}}$. By Lemma 54, letting

$$
\gamma_{i}: 0 \rightarrow \mathcal{G}_{2} \otimes \mathcal{O}_{T} \rightarrow \mathcal{Q}_{i} \rightarrow \mathcal{G}_{1} \otimes \mathcal{O}_{T} \rightarrow 0
$$

be the extensions given by $\gamma_{i}:=q_{i}\left(\beta_{1}, \ldots, \beta_{t}\right)$ for $i=1, \ldots, u$, we have

$$
\sigma_{\beta_{c}}=\sum_{i=1}^{u} p_{i}\left(c_{1}, \ldots, c_{t}\right) \sigma_{\gamma_{i}} .
$$


Applying $\zeta_{\alpha}^{-1}$, we get

$$
\theta_{\mathcal{F}_{c} \mid \Sigma}=\sum_{i=1}^{u} p_{i}\left(c_{1}, \ldots, c_{t}\right) \theta_{\mathcal{Q}_{i} \mid \Sigma}
$$

Proposition 56. For every integer $m \geq 0$ the following statements hold.

(1) $A_{\mathcal{E} \mid \Sigma}^{m}$ is coherent.

(2) If $\Sigma^{\prime} \subseteq \Sigma$ is an open subspace, there is a natural surjection $A_{\mathcal{E} \mid \Sigma}^{m} \rightarrow A_{\mathcal{E} \mid \Sigma^{\prime}}^{m}$.

(3) For each map $q: S^{\prime} \rightarrow S$ there is a base-change map $\nu_{q}: q^{*} A_{\mathcal{E} \mid \Sigma}^{m} \rightarrow A_{\mathcal{E}^{\prime} \mid \Sigma^{\prime}}^{m}$, where $\Sigma^{\prime}:=\Sigma \times S^{\prime}$ and $\mathcal{E}^{\prime}:=\mathcal{E} \otimes \mathcal{O}_{S^{\prime}}$. If $q$ is flat, then $\nu_{q}$ is an isomorphism.

Proof. Statement (2) is clear from the fact that theta functions are already defined on $J_{d}$ (see Subsection 6.2).

We will prove (3) and show that $A_{\mathcal{E} \mid \Sigma}^{m}$ is quasi-coherent. Let $q: S^{\prime} \rightarrow S$ be a map, $\Sigma^{\prime}:=\Sigma \times S^{\prime}$ and $\mathcal{E}^{\prime}:=\mathcal{E} \otimes \mathcal{O}_{S^{\prime}}$. Let $\pi^{\prime}: \Sigma^{\prime} \rightarrow S^{\prime}$ be the structure map. It follows from the base-change property of the determinant of cohomology that the natural composition

$$
q^{*} A_{\mathcal{E} \mid \Sigma}^{m} \rightarrow q^{*} \pi_{*}\left(\mathcal{L}_{\mathcal{E} \mid \Sigma}^{\otimes m}\right) \rightarrow \pi_{*}^{\prime}\left(\mathcal{L}_{\mathcal{E}^{\prime} \mid \Sigma^{\prime}}^{\otimes m}\right)
$$

factors through $A_{\mathcal{E}^{\prime} \mid \Sigma^{\prime}}^{m}$, yielding the map $\nu_{q}$ of (3). If $q$ is flat, since $\pi$ is of finite type, $q^{*} \pi_{*}\left(\mathcal{L}_{\mathcal{E} \mid \Sigma}^{\otimes m}\right) \rightarrow \pi_{*}^{\prime}\left(\mathcal{L}_{\mathcal{E}^{\prime} \mid \Sigma^{\prime}}^{\otimes m}\right)$ is an isomorphism. So, $\nu_{q}$ is injective.

For all flat maps $q: S^{\prime} \rightarrow S$ and all affine open subschemes $U \subseteq S$ and $U^{\prime} \subseteq S^{\prime}$ such that $q\left(U^{\prime}\right) \subseteq U$, consider the natural diagram of maps

$$
\begin{array}{ccc}
A_{\mathcal{E} \mid \Sigma}^{m}(U) \otimes H^{0}\left(U^{\prime}, \mathcal{O}_{S^{\prime}}\right) & \stackrel{\mu_{q, U, U^{\prime}}}{\longrightarrow} & A_{\mathcal{E}^{\prime} \mid \Sigma^{\prime}}^{m}\left(U^{\prime}\right) \\
\downarrow & & \\
H^{0}\left(U, \pi_{*} \mathcal{L}_{\mathcal{E} \mid \Sigma}^{\otimes m}\right) \otimes H^{0}\left(U^{\prime}, \mathcal{O}_{S^{\prime}}\right) & \rightarrow & H^{0}\left(U^{\prime}, \pi_{*}^{\prime}\left(\mathcal{L}_{\mathcal{E}^{\prime} \mid \Sigma^{\prime}}^{\otimes m}\right)\right)
\end{array}
$$

Since $\pi$ is of finite type and $q$ is flat, the bottom map is an isomorphism. So, $\mu_{q, U, U^{\prime}}$ is injective. We claim $\mu_{q, U, U^{\prime}}$ is surjective as well.

If we show that $\mu_{1_{S}, U, U_{b}}$ is surjective for every affine open subscheme $U \subseteq S$ and every regular function $b$ on $U$, it follows that $A_{\mathcal{E} \mid \Sigma}^{m}$ is quasi-coherent. As for (3), if $A_{\mathcal{E} \mid \Sigma}^{m}$ is quasi-coherent, then $\mu_{q, U, U^{\prime}}=\nu_{q}\left(U^{\prime}\right)$ for all affine open subschemes $U \subseteq S$ and $U^{\prime} \subseteq S^{\prime}$ such that $q\left(U^{\prime}\right) \subseteq U$. Since $\nu_{q}\left(U^{\prime}\right)$ is injective, if we show that $\mu_{q, U, U^{\prime}}$ is surjective for all $U$ and $U^{\prime}$ as above, it follows that $\nu_{q}$ is an isomorphism. So, it is enough to prove the claim. Up to changing the notation, we may assume $U=S$ and $U^{\prime}=S^{\prime}$, and let $\mu_{q}:=\mu_{q, S, S^{\prime}}$.

Let $\mathcal{O}_{X}(1)$ be an ample sheaf on $X / S$. Since $A_{\mathcal{E} \mid \Sigma}^{m}$ (resp. $\left.A_{\mathcal{E}^{\prime} \mid \Sigma^{\prime}}^{m}\right)$ is an étale sheaf, we may replace $S$ (resp. $S^{\prime}$ ) by an affine, étale covering, if necessary. In particular, we may assume there is a universal torsion-free, rank-1 sheaf $\mathcal{I}$ on $X \times \Sigma / \Sigma$. Since $S$ is Noetherian, and $\pi$ is of finite type, there is an integer $c_{0}$ such that, for every $c \geq c_{0}$ and every $s \in \Sigma$,

$$
h^{1}\left(X(s), \mathcal{I}(s) \otimes \mathcal{N}_{c}(s)\right)=h^{0}\left(X(s), \mathcal{I}(s) \otimes \mathcal{B}_{c}(s)\right)=0,
$$

where $\mathcal{N}_{c}:=(\operatorname{det} \mathcal{E})^{\otimes m}(c(m r-1))$ and $\mathcal{B}_{c}:=\mathcal{O}_{X}(-c)^{\oplus m r-1}$.

Let $f^{\prime}: X \times S^{\prime} \rightarrow S^{\prime}$ denote the projection. Let $\theta \in A_{\mathcal{E}^{\prime} \mid \Sigma^{\prime}}^{m}\left(S^{\prime}\right)$. We claim that $\theta \in \operatorname{im}\left(\mu_{q}\right)$. Up to replacing $S^{\prime}$ by an affine, étale covering, we may assume $\theta=a_{1} \theta_{\mathcal{G}_{1}}+\cdots+a_{t} \theta_{\mathcal{G}_{t}}$, where $a_{1}, \ldots, a_{t} \in H^{0}\left(S^{\prime}, \mathcal{O}_{S^{\prime}}\right)$, where $\mathcal{G}_{1}, \ldots, \mathcal{G}_{t}$ are vector bundles of rank $m r$ on $X \times S^{\prime}$, and $\theta_{\mathcal{G}_{1}}, \ldots, \theta_{\mathcal{G}_{t}}$ are constructed from isomorphisms 
$\phi_{i}: \operatorname{det} \mathcal{G}_{i} \cong(\operatorname{det} \mathcal{E})^{\otimes m} \otimes \mathcal{O}_{S^{\prime}}$ and $\psi_{i}: \mathcal{D}_{f}(\mathcal{E})^{\otimes m} \otimes \mathcal{O}_{S^{\prime}} \cong \mathcal{D}_{f^{\prime}}\left(\mathcal{G}_{i}\right)$ for $i=1, \ldots, t$. By Lemma 17, up to replacing $S$ and $S^{\prime}$ by affine, étale coverings, we may assume there are an integer $c \geq c_{0}$ and extensions

$$
\begin{gathered}
\alpha: 0 \rightarrow \mathcal{B}_{c} \rightarrow \mathcal{E}^{\oplus m} \rightarrow \mathcal{N}_{c} \rightarrow 0, \\
\beta_{i}: 0 \rightarrow \mathcal{B}_{c} \otimes \mathcal{O}_{S^{\prime}} \rightarrow \mathcal{G}_{i} \rightarrow \mathcal{N}_{c} \otimes \mathcal{O}_{S^{\prime}} \rightarrow 0
\end{gathered}
$$

for $i=1, \ldots, t$. Fix such $c$. We may choose the isomorphisms $\phi_{i}$ and $\psi_{i}$ such that $\left(\operatorname{det}(\alpha) \otimes \mathcal{O}_{S^{\prime}}\right) \phi_{i}=\operatorname{det}\left(\beta_{i}\right)$ and $\mathcal{D}_{f}(\alpha) \otimes \mathcal{O}_{S^{\prime}}=\mathcal{D}_{f^{\prime}}\left(\beta_{i}\right) \psi_{i}$ for $i=1, \ldots, t$.

Since the fibers of $f$ are curves, the formation of $\operatorname{Ext}_{f}^{1}\left(\mathcal{N}_{c}, \mathcal{B}_{c}\right)$ commutes with base change. In particular,

$$
\operatorname{Ext}_{X}^{1}\left(\mathcal{N}_{c}, \mathcal{B}_{c}\right) \otimes H^{0}\left(S^{\prime}, \mathcal{O}_{S^{\prime}}\right)=\operatorname{Ext}_{X \times S^{\prime}}^{1}\left(\mathcal{N}_{c} \otimes \mathcal{O}_{S^{\prime}}, \mathcal{B}_{c} \otimes \mathcal{O}_{S^{\prime}}\right)
$$

Thus, we may write $\beta_{i}=\sum_{j} b_{i, j} \gamma_{i, j}$ for $i=1, \ldots, t$, where $b_{i, j} \in H^{0}\left(S^{\prime}, \mathcal{O}_{S^{\prime}}\right)$ and $\gamma_{i, j} \in \operatorname{Ext}_{X}^{1}\left(\mathcal{N}_{c}, \mathcal{B}_{c}\right)$ for all $j$. Since (56.1) holds, by Construction 55, up to replacing $S$ by an affine, étale covering, there are extensions

$$
0 \rightarrow \mathcal{B}_{c} \rightarrow \mathcal{F}_{i, j} \rightarrow \mathcal{N}_{c} \rightarrow 0
$$

such that $\theta_{\mathcal{G}_{i}}=\sum_{j} c_{i, j} \theta_{\mathcal{F}_{i, j}}$ for $i=1, \ldots, t$, with $c_{i, j} \in H^{0}\left(S^{\prime}, \mathcal{O}_{S^{\prime}}\right)$. Using that $\theta=\sum_{i} a_{i} \theta_{\mathcal{G}_{i}}$, we have $\theta \in \operatorname{im}\left(\mu_{q}\right)$, as wished.

We will show now that $A_{\mathcal{E} \mid \Sigma}^{m}$ is coherent. We may assume $S$ is Noetherian. Since $A_{\mathcal{E} \mid \Sigma}^{m}$ is quasi-coherent with formation commuting with étale base change, we may replace $S$ by an étale covering. By Lemmas 18 and 35, since $\Sigma$ is Noetherian, we may thus assume there are a section $\sigma: S \rightarrow X$ of $f$ through the $S$-smooth locus of $X$ and polarizations $\mathcal{H}_{1}, \ldots, \mathcal{H}_{t}$ on $X / S$, such that

$$
\Sigma \subseteq \Sigma^{\prime}:=\bigcup_{j=1}^{t} J_{\mathcal{H}_{i}}^{\sigma}
$$

Let $\pi^{\prime}: \Sigma^{\prime} \rightarrow S$ be the structure map. By Theorem A, the spaces $J_{\mathcal{H}_{i}}^{\sigma}$ are proper over $S$. As in Remark 53, the sheaf $\pi_{*}^{\prime} \mathcal{L}_{\mathcal{E} \mid \Sigma^{\prime}}^{\otimes m}$ is coherent, and thus so is $A_{\mathcal{E} \mid \Sigma^{\prime}}^{m}$. Since there is a surjection $A_{\mathcal{E} \mid \Sigma^{\prime}}^{m} \rightarrow A_{\mathcal{E} \mid \Sigma}^{m}$, we get that $A_{\mathcal{E} \mid \Sigma}^{m}$ is coherent as well.

Let

$$
V_{\mathcal{E} \mid \Sigma}:=\bigoplus_{m} A_{\mathcal{E} \mid \Sigma}^{m} \subseteq \Gamma_{\mathcal{E} \mid \Sigma}:=\bigoplus_{m} \pi_{*} \mathcal{L}_{\mathcal{E} \mid \Sigma}^{\otimes m}
$$

It follows from the additive property of the determinant of cohomology and the additive property of the isomorphism $\tau$ of Lemma 46 [12, Thm. I.1, p. 509] that $V_{\mathcal{E} \mid \Sigma}$ is a graded subsheaf of $\mathcal{O}_{S}$-algebras of $\Gamma_{\mathcal{E} \mid \Sigma}$. We say that $V_{\mathcal{E} \mid \Sigma}$ is the sheaf of theta functions on $\Sigma$ with respect to $\mathcal{E}$. Put $\bar{\Sigma}_{\mathcal{E}}:=\operatorname{Proj}\left(V_{\mathcal{E} \mid \Sigma}\right)$. By Proposition 56, (1), the scheme $\bar{\Sigma}_{\mathcal{E}}$ is separated over $S$. Let $\psi: \Sigma \rightarrow \bar{\Sigma}_{\mathcal{E}}$ denote the natural rational map.

Proof of Theorem C. Statements (1)-(3) of Theorem 48 hold when we replace $\tilde{\Sigma}_{\mathcal{E}}$ by $\bar{\Sigma}_{\mathcal{E}}$. In fact, the same proofs can be given. (We need the formation of $\bar{\Sigma}_{\mathcal{E}}$ to commute with flat base change, a fact guaranteed by Proposition 56, (3).) So, we have (2), (4), and that the fibers of $\psi(s)$ are contained in the JH-equivalence classes of $\Sigma(s)$ for every $s \in S$. 
Let $s \in S$. We will show that a JH-equivalence class of $\Sigma(s)$ is contained in a fiber of $\psi(s)$. Let $t_{1}, t_{2} \in \Sigma(s)$. For $i=1,2$ there are a finite and separable field extension $k_{i} \supseteq k\left(t_{i}\right)$ and a semi-stable sheaf $I_{i}$ in $X\left(k_{i}\right)$ with respect to $\mathcal{E}\left(k_{i}\right)$ representing $t_{i}$. Let $k \supseteq k(s)$ be an algebraically closed field extension containing $k_{1}$ and $k_{2}$. Assume $\operatorname{Gr}\left(I_{1}(k)\right) \cong \operatorname{Gr}\left(I_{2}(k)\right)$. We need to show that $\psi\left(t_{1}\right)=\psi\left(t_{2}\right)$. By Proposition 56, (3), the map $\psi(k): \Sigma(k) \rightarrow \bar{\Sigma}_{\mathcal{E}}(k)$ factors through the natural map $\Sigma(k) \rightarrow \overline{\Sigma(k)}_{\mathcal{E}(k)}$. We may thus assume $k=k(s)$ and $S:=\operatorname{Spec}(k)$.

Let $m$ be an integer, and $F_{1}, F_{2}$ vector bundles on $X$ of rank $m r$ and determinant $(\operatorname{det} \mathcal{E})^{\otimes m}$ such that

$$
h^{0}\left(X, I_{1} \otimes F_{2}\right)=h^{1}\left(X, I_{1} \otimes F_{2}\right)=0 .
$$

Tensoring a Jordan-Hölder filtration of $I_{1}$ with $F_{2}$, and considering the associated long exact sequences in cohomology, we get

$$
h^{0}\left(X, \operatorname{Gr}\left(I_{1}\right) \otimes F_{2}\right)=h^{1}\left(X, \operatorname{Gr}\left(I_{1}\right) \otimes F_{2}\right)=0 .
$$

By the same argument, since $\operatorname{Gr}\left(I_{1}\right)=\operatorname{Gr}\left(I_{2}\right)$,

$$
h^{0}\left(X, I_{2} \otimes F_{2}\right)=h^{1}\left(X, I_{2} \otimes F_{2}\right)=0
$$

as well. It will be enough to show that

$$
\frac{\theta_{F_{1}}}{\theta_{F_{2}}}\left(t_{1}\right)=\frac{\theta_{F_{1}}}{\theta_{F_{2}}}\left(t_{2}\right)
$$

By Lemma 17, there are vector bundles $H_{1}$ and $H_{2}$ on $X$ and extensions

$$
\begin{aligned}
& \alpha: 0 \rightarrow H_{2} \rightarrow E^{\oplus m} \rightarrow H_{1} \rightarrow 0, \\
& \beta_{i}: 0 \rightarrow H_{2} \rightarrow F_{i} \rightarrow H_{1} \rightarrow 0
\end{aligned}
$$

for $i=1,2$. Moreover, since $\Sigma$ is Noetherian, we may choose $H_{1}, H_{2}$ such that

$$
h^{0}\left(X(s), \mathcal{I}(s) \otimes H_{2}(s)\right)=h^{1}\left(X(s), \mathcal{I}(s) \otimes H_{1}(s)\right)=0
$$

for every $s \in \Sigma$, where $\mathcal{I}$ is a universal sheaf on $X \times \Sigma$.

Let $p_{1}, p_{2}$ denote the projections from $X \times \mathbf{A}_{k}^{2}$ onto the indicated factors. Consider the extension

$$
\beta: 0 \rightarrow p_{1}^{*} H_{2} \rightarrow \mathcal{F} \rightarrow p_{1}^{*} H_{1} \rightarrow 0
$$

on $X \times \mathbf{A}_{k}^{2}$ whose restriction to the fiber $X(a)$ is $\beta_{a}:=a_{1} \beta_{1}+a_{2} \beta_{2}$ for every $k$-point $a:=\left(a_{1}, a_{2}\right) \in \mathbf{A}_{k}^{2}$. Let $D_{i} \subseteq \mathbf{A}_{k}^{2}$ denote the zero-scheme of $\sigma_{p_{1}^{*} I_{i} \otimes \mathcal{F}}$ for $i=1,2$. Since $\operatorname{Gr}\left(I_{1}\right) \cong \operatorname{Gr}\left(I_{2}\right)$, considering the Jordan-Hölder filtrations of $I_{1}$ and $I_{2}$, and using the additive property of the determinant of cohomology, we have $D_{1}=D_{2}$. By Lemma 54 and Construction 55, there are polynomials $p_{1}, \ldots, p_{u} \in k\left[T_{1}, T_{2}\right]$ and $k$-points $\left(b_{1}, c_{1}\right), \ldots,\left(b_{u}, c_{u}\right) \in \mathbf{A}_{k}^{2}$ such that

$$
\theta_{\mathcal{F}(a)}=\sum_{i=1}^{u} p_{i}\left(a_{1}, a_{2}\right) \theta_{G_{i}}
$$

for every $k$-point $a:=\left(a_{1}, a_{2}\right) \in \mathbf{A}_{k}^{2}$, where $G_{i}:=\mathcal{F}\left(b_{i}, c_{i}\right)$ for $i=1, \ldots, u$. Consider the polynomials

$$
q_{j}\left(T_{1}, T_{2}\right):=\sum_{i=1}^{u} \frac{\theta_{G_{i}}}{\theta_{F_{2}}}\left(t_{j}\right) p_{i}\left(T_{1}, T_{2}\right)
$$


for $j=1,2$. Since $D_{j}$ is the zero-scheme of $q_{j}$ for $j=1,2$, there is $\lambda \in k^{*}$ such that $q_{1}=\lambda q_{2}$. Hence,

$$
\frac{\theta_{\mathcal{F}(a)}}{\theta_{F_{2}}}\left(t_{1}\right)=\lambda \frac{\theta_{\mathcal{F}(a)}}{\theta_{F_{2}}}\left(t_{2}\right)
$$

for every $k$-point $a \in \mathbf{A}_{k}^{2}$. Letting $a:=(0,1)$ we get $\lambda=1$. Letting $a:=(1,0)$ we get

$$
\frac{\theta_{F_{1}}}{\theta_{F_{2}}}\left(t_{1}\right)=\frac{\theta_{F_{1}}}{\theta_{F_{2}}}\left(t_{2}\right),
$$

as wished.

It remains to show (1). For every $m \geq 0$, denote by $V_{\mathcal{E} \mid \Sigma}(m)$ the graded subsheaf of $\mathcal{O}_{S^{-}}$-algebras of $V_{\mathcal{E} \mid \Sigma}$ generated by the homogeneous pieces of $V_{\mathcal{E} \mid \Sigma}$ of degree at most $m$. Let $\bar{\Sigma}_{\mathcal{E}}(m):=\operatorname{Proj}\left(V_{\mathcal{E} \mid \Sigma}(m)\right)$ for every $m \geq 0$. By Proposition 56, (1), the scheme $\bar{\Sigma}_{\mathcal{E}}(m)$ is locally projective over $S$ for every $m \geq 0$. The proofs of (2)-(4) hold for the natural rational map $\psi_{m}: \Sigma \rightarrow \bar{\Sigma}_{\mathcal{E}}(m)$ in place of $\psi$, as long as $m>>0$. (In fact, as in Remark 49, as long as $m>\max (e(X / S), 2)$.) By Proposition 56, (2), there is a natural closed embedding $\bar{\Sigma}_{\mathcal{E}} \hookrightarrow \overline{J_{\mathcal{E}}^{s s}}$. We may thus assume $\Sigma:=J_{\mathcal{E}}^{s s}$. Since $J_{\mathcal{E}}^{s s}$ is universally closed over $S$, and $\psi_{m}$ is dominant by (2), it follows that $\psi_{m}$ is surjective for $m>>0$. In addition, by (3), the natural maps $\alpha_{m}: \bar{\Sigma}_{\mathcal{E}}(m+1) \rightarrow \bar{\Sigma}_{\mathcal{E}}(m)$ are injective for $m>>$. Since $\bar{\Sigma}_{\mathcal{E}}(m)$ is locally projective over $S$ for every $m$, the map $\alpha_{m}$ is proper, hence finite for $m>>0$. Since the natural maps $\psi_{m}: \Sigma \rightarrow \bar{\Sigma}_{\mathcal{E}}(m)$ are defined and scheme-theoretically dominant for $m>>0$, it follows that $\bar{\Sigma}_{\mathcal{E}}$ is the normalization of $\bar{\Sigma}_{\mathcal{E}}(m)$ under $\psi_{m}$ for every $m>>0$. Since $S$ is excellent, $\bar{\Sigma}_{\mathcal{E}}$ is finite over $\bar{\Sigma}_{\mathcal{E}}(m)$ for $m>>0$. Since the $\bar{\Sigma}_{\mathcal{E}}(m)$ are locally projective over $S$, so is $\bar{\Sigma}_{\mathcal{E}}$.

\section{Comparison With Seshadri's COMPACTIFICATION}

Let $X$ be a curve over an algebraically closed field $k$. Let $X_{1}, \ldots, X_{n}$ denote the irreducible components of $X$. Let $\underline{a}:=\left(a_{1}, \ldots, a_{n}\right)$ be an $n$-uple of positive rational numbers such that $a_{1}+\cdots+a_{n}=1$. According to Seshadri's definition [25, Part 7], a torsion-free, rank-1 sheaf $I$ on $X$ is called $\underline{a}$-semi-stable (resp. $\underline{a}$-stable) if and only if

$$
\chi\left(I_{Y}\right) \geq\left(a_{i_{1}}+\cdots+a_{i_{m}}\right) \chi(I) \quad\left(\operatorname{resp} . \quad \chi\left(I_{Y}\right)>\left(a_{i_{1}}+\cdots+a_{i_{m}}\right) \chi(I)\right)
$$

for every non-empty, proper subcurve $Y=X_{i_{1}} \cup \cdots \cup X_{i_{m}} \varsubsetneqq X$. (Actually, Seshadri defined the notions of $\underline{a}$-stability and $\underline{a}$-semi-stability for any torsion-free sheaf on $X$, not necessarily rank-1.)

Observation 5\%. We shall observe that Seshadri's notions of semi-stability and stability are equivalent to ours. Let $d$ be a positive integer. Let $\underline{a}$ be a $n$-uple of positive rational numbers such that $a_{1}+\cdots+a_{n}=1$. Let $\underline{A}:=\left(A_{1}, \ldots, A_{n}\right)$ be a $n$-uple of positive integers such that $A:=A_{1}+\cdots+A_{n}$ is a multiple of $d$ and $A_{i} a_{j}=A_{j} a_{i}$ for $1 \leq i, j \leq n$. Since $a_{1}+\cdots+a_{n}=1$, it follows that $a_{i}:=A_{i} / A$ for $i=1, \ldots, n$. For each $i=1, \ldots, n$, let $x_{1}^{i}, \ldots, x_{A_{i}}^{i} \in X_{i}$ be distinct, non-singular $k$-points of $X$. Let

$$
\mathcal{O}_{X}(1):=\mathcal{O}_{X}\left(\sum_{\substack{1 \leq i \leq n \\ 1 \leq j \leq A_{i}}} x_{j}^{i}\right)
$$


Of course, $\mathcal{O}_{X}(1)$ is an ample sheaf on $X$. Let

$$
E:=\left(\mathcal{O}_{X}^{\oplus(r / t-1)} \oplus \mathcal{O}_{X}(-1)\right)^{\oplus t}
$$

for a certain integer $t>0$, to be specified later, and $r:=t A / d$. As observed in [10] Obs. 13], a torsion-free, rank-1 sheaf $I$ on $X$ with $\chi(I)=d$ is $\underline{a}$-semi-stable (resp. $\underline{a}$-stable) if and only if $I$ is semi-stable (resp. stable) with respect to $E$.

Let $S(\underline{a}, d)$ (resp. $\left.S^{\prime}(\underline{a}, d)\right)$ be the set of isomorphism classes of $\underline{a}$-semi-stable (resp. $\underline{a}$-stable) torsion-free, rank-1 sheaves of Euler characteristic $d$ on $X$.

Theorem 58 (Seshadri). There is a coarse moduli space for $S^{\prime}(\underline{a}, d)$, whose underlying scheme is a quasi-projective variety denoted by $U^{s}(\underline{a}, d)$. Moreover, $U^{s}(\underline{a}, d)$ has a natural projective compactification, denoted by $U(\underline{a}, d)$. The set of closed points of $U(\underline{a}, d)$ is isomorphic to the quotient of $S(\underline{a}, d)$ by the JH-equivalence relation.

Proof. See [25, Thm. 15, p. 155].

Seshadri constructed $U(\underline{a}, d)$ as a good quotient $\phi: R \rightarrow U(\underline{a}, d)$ of a quasiprojective scheme $R$ under the action of a reductive group, using Geometric Invariant Theory. In Seshadri's construction, there is a torsion-free, rank-1 sheaf $\mathbf{I}$ on $X \times R / R$ such that $\mathbf{I}$ has the local universal property for $S(\underline{a}, d)$. Let $p_{1}, p_{2}$ denote the projections from $X \times R$ onto the indicated factors. If we take the polarization $E$ in (57.1) with $t>>0$, then the determinant of cohomology $\mathcal{D}_{p_{2}}\left(\mathbf{I} \otimes p_{1}^{*} E\right)$ descends to an ample invertible sheaf $\bar{L}_{E}$ on $U(\underline{a}, d)$ (see [10, Thm. 14] and the argument thereafter). In addition, for every integer $m$ and every vector bundle $F$ on $X$ with rank $m r$ and $\operatorname{det} F \cong(\operatorname{det} E)^{\otimes m}$, the section $\sigma_{\mathbf{I} \otimes p_{1}^{*} F} \in H^{0}\left(R, \mathcal{D}_{p_{2}}\left(\mathbf{I} \otimes p_{1}^{*} F\right)\right)$ corresponds in a natural way (modulo $k^{*}$ ) to a section of $\mathcal{D}_{p_{2}}\left(\mathbf{I} \otimes p_{1}^{*} E\right)^{\otimes m}$ (as in Subsection 6.2) that descends to a section of $\bar{L}_{E}^{\otimes m}$. We shall denote by $\bar{\theta}_{F}$ the descended section. We emphasize that $\bar{\theta}_{F}$ is well defined modulo $k^{*}$. Let

$$
\bar{\Gamma}_{E}:=\bigoplus_{m \geq 0} H^{0}\left(U(\underline{a}, d), \bar{L}_{E}^{\otimes m}\right),
$$

and $\bar{V}_{E} \subseteq \bar{\Gamma}_{E}$ denote the graded $k$-subalgebra generated by the sections $\bar{\theta}_{F}$. Let $\bar{U}(\underline{a}, d):=\operatorname{Proj}\left(\bar{V}_{E}\right)$.

Theorem 59. The rational map $\pi: U(\underline{a}, d) \rightarrow \bar{U}(\underline{a}, d)$ is defined everywhere, bijective, and an isomorphism on $U^{s}(\underline{a}, d)$.

Proof. See [10, Thm. 16]. Alternatively, the theorem can be proved with the same arguments used in the proof of Theorem 48.

Let $J_{E}^{s s}$ be the fine moduli space of semi-stable sheaves on $X$ with respect to $E$ (see Section 4). Let

$$
\psi: J_{E}^{s s} \rightarrow \overline{J_{E}^{s s}}
$$

be the naturally defined rational map (see Subsection 7.2). Since $J_{E}^{s s}$ is equipped with a universal sheaf, there is a naturally defined map

$$
\rho: J_{E}^{s s} \rightarrow U(\underline{a}, d),
$$

sending the point in $J_{E}^{s s}$ represented by a torsion-free, rank-1 sheaf $I$ to the point in $U(\underline{a}, d)$ represented by $\operatorname{Gr}(I)$. So, $\rho^{-1}\left(U^{s}(\underline{a}, d)\right)=J_{E}^{s}$. Moreover, since $J_{E}^{s}$ 
and $U^{s}(\underline{a}, d)$ are respectively fine and coarse moduli spaces for the same moduli problem, $\left.\rho\right|_{J_{E}^{s}}: J_{E}^{s} \rightarrow U^{s}(\underline{a}, d)$ is an isomorphism.

Let $L_{E}$ be the theta line bundle on $J_{E}^{s s}$. Since $\mathbf{I}$ has the local universal property for $S(\underline{a}, d)$, and there is a universal sheaf $\mathcal{I}$ on $X \times J_{\mathcal{E}}^{s s}$, there are an affine, open covering $J_{\mathcal{E}}^{s s}=\bigcup_{i} U_{i}$, maps $\rho_{i}: U_{i} \rightarrow R$ and isomorphisms $h_{i}:\left.\mathcal{I}\right|_{X \times U_{i}} \cong \mathbf{I} \otimes \mathcal{O}_{U_{i}}$ for every $i$. Let $\lambda_{i}:\left.\left.L_{E}\right|_{U_{i}} \cong \rho^{*} \bar{L}_{E}\right|_{U_{i}}$ be the isomorphism induced by $h_{i}$ for every $i$. As $\mathcal{I}$ is simple, for all $i, j$ there are an open covering $U_{i} \cap U_{j}=\bigcup_{l} U_{i, j, l}$ and regular, invertible functions $g_{i, j, l}$ on $U_{i, j, l}$ such that $\left.h_{i}\right|_{X \times U_{i, j, l}}=\left.g_{i, j, l} h_{j}\right|_{X \times U_{i, j, l}}$ for every $l$. Since $\chi\left(\mathbf{I} \otimes p_{1}^{*} E / R\right)=0$, by the functorial property of the determinant

of cohomology, $\left.\lambda_{i}\right|_{U_{i, j, l}}=\left.\lambda_{j}\right|_{U_{i, j, l}}$ for all $i, j, l$. Thus, the $\lambda_{i}$ patch to a global isomorphism $\lambda: L_{E} \cong \rho^{*} \bar{L}_{E}$. Using a similar argument, we can also show that $\rho^{*} \bar{\theta}_{F}=\lambda \theta_{F}\left(\right.$ modulo $\left.k^{*}\right)$ for every integer $m$ and every vector bundle $F$ on $X$ of rank $m r$ and determinant $(\operatorname{det} E)^{\otimes m}$.

Let $\Sigma \subseteq J_{\mathcal{E}}^{s s}$ be an open subscheme. Let $V_{E}$ denote the ring of theta functions on $\Sigma$ (see Subsection 7.2). Since $V_{E}$ is generated by the theta functions $\theta_{F}$ and $\bar{V}_{E}$ by the $\bar{\theta}_{F}$, the isomorphism $\lambda$ induces a surjective, graded map of $k$-algebras $\bar{V}_{E} \rightarrow V_{E}$, and hence a closed embedding

$$
\iota: \bar{\Sigma}_{\mathcal{E}} \hookrightarrow \bar{U}(\underline{a}, d) .
$$

It is clear that $\left.\pi \circ \rho\right|_{\Sigma}=\left.\iota \circ \psi\right|_{\Sigma}$.

Theorem 60. If the singularities of $X$ are ordinary double points, and $\Sigma \supseteq J_{E}^{p}$ for a non-singular $p \in X$, then $\iota: \bar{\Sigma} \cong \bar{U}(\underline{a}, d)$ is an isomorphism.

Proof. If $\Sigma \supseteq J_{E}^{p}$ for a non-singular $p \in X$, then it follows from Theorem 7 that $\left.\rho\right|_{\Sigma}$ is surjective. Since $\pi$ is bijective, so is $\iota$. If $U(\underline{a}, d)$ were reduced, then $\iota$ would be an isomorphism. If $X$ has at most ordinary double points for singularities, then $U(\underline{a}, d)$ is known to be reduced [1, Cor. 3.5].

If we had a better understanding of the tangent spaces at semi-stable points of $U(\underline{a}, d)$, then we could probably say more about the maps $\pi$ and $\iota$.

\section{ACKNOWLEDGEMENTS}

I take the opportunity to thank Professor S. Kleiman for introducing me to the compactification problem and for several comments on different versions of the present article. I am also grateful to Professor L. Ramero for telling me about Faltings' construction of the moduli space of semi-stable vector bundles on a smooth curve via theta functions, and Professor T. Kajiwara for various comments. I must thank the referee for invaluable suggestions that greatly improved the presentation. I would like to thank Waseda University, and especially Professors H. Kaji, T. Morimoto, M. Ohno and N. Hara, for the warm hospitality extended during the period the essential part of this work was done. I am also grateful to Professor M. Homma for all the help received from him during the said period and the proof of Lemma 54 .

\section{REFERENCES}

1. V. Alexeev, Compactified Jacobians, Available at http://xxx.lanl.gov/abs/alg-geom/9608012 August, 1996. 
2. A. Altman, A. Iarrobino and S. Kleiman, Irreducibility of the compactified Jacobian, Real and complex singularities, Oslo 1976 (Proc. Ninth Nordic Summer School), Sijthoff and Noordhoff, 1977, pp. 1-12. MR 58:16650

3. A. Altman and S. Kleiman, Compactifying the Picard scheme, Adv. Math. 35 (1980), 50-112. MR 81f:14025a

4. E. Arbarello, M. Cornalba, P. Griffiths and J. Harris, Geometry of algebraic curves, Vol. I, Grundlehren der mathematischen Wissenschaften, vol. 267, Springer-Verlag, 1985. MR 86h:14019

5. M. Artin, Algebraization of formal moduli. I, Global Analysis, Papers in honor of K. Kodaira, University of Tokyo Press, Princeton University Press, 1969, pp. 21-71. MR 41:5369

6. S. Bosch, W. Lütkebohmert, and M. Raynaud, Néron models, Ergebnisse der Mathematik und ihrer Grenzgebiete, vol. 21, Springer Verlag, 1990. MR 91i:14034

7. L. Caporaso, A compactification of the universal Picard variety over the moduli space of stable curves, J. Amer. Math. Soc. 7 (1994), 589-660. MR 95d:14014

8. C. D'Souza, Compactification of generalized Jacobians, Proc. Indian Acad. Sci. Sect. A Math. Sci. 88 (1979), 419-457. MR 81h:14004

9. E. Esteves, Very ampleness for Theta on the compactified Jacobian, Math. Z. 226 (2) (1997), 181-191. MR 98k:14037

10. E. Esteves, Separation properties of theta functions, Duke Math. J. 98 (3) (1999), 565-593. MR 2000e: 14048

11. E. Esteves, M. Gagné and S. Kleiman, Abel maps and presentation schemes, Available at http://xxx.lanl.gov/abs/math.AG/9911069 November, 1999. To appear in a special issue, dedicated to R. Hartshorne.

12. G. Faltings, Stable G-bundles and projective connections, J. Algebraic Geom. 2 (1993), 507568. MR 94i:14015

13. D. Gieseker, Moduli of curves, Tata Inst. Fund. Res. Lecture Notes, Springer-Verlag, 1982.

14. A. Grothendieck with J. Dieudonné, Éleménts de Géométrie Algébrique III-1, IV-4, Inst. Hautes Études Sci. Publ. Math. 11, 32 (1961, 1967). MR 36:177c; MR 39:220

15. A. Grothendieck, Fondements de la géometrie algébrique, Semináire Bourbaki, exp. 232 (1961/62). MR 26:3566

16. M. Homma, Personal communication.

17. J. Igusa, Fibre systems of Jacobian varieties, Amer. J. Math. 78 (1956), 171-199. MR 18:935d

18. L. Illusie, Conditions de finitude relatives, Lecture Notes in Mathematics, vol. 225, SpringerVerlag, 1971, pp. 222-273.

19. M. Ishida, Compactifications of a family of generalized Jacobian varieties, Proc. Internat. Sympos. Algebraic Geometry, Kyoto, January, 1977 (M. Nagata, ed.), Kinokuniya, Tokyo, 1978, pp. 503-524. MR 81h:14025

20. F. Knudsen and D. Mumford, The projectivity of the moduli space of stable curves I: Preliminaries on "det" and "Div", Math. Scand. 39 (1976), 19-55. MR 55:10465

21. S. Langton, Valuative criteria for families of vector bundles on algebraic varieties, Ann. Math. 101 (1975), 88-110. MR 51:510

22. A. Mayer and D. Mumford, Further comments on boundary points, Unpublished lecture notes distributed at the Amer. Math. Soc. Summer Institute, Woods Hole, 1964.

23. T. Oda and C.S. Seshadri, Compactifications of the generalized Jacobian variety, Trans. Amer. Math. Soc. 253 (1979), 1-90. MR 82e:14054

24. R. Pandharipande, A compactification over $\bar{M}_{g}$ of the universal moduli space of slope-semistable vector bundles, J. Amer. Math. Soc. 9 (1996), 425-471. MR 96f:14014

25. C.S. Seshadri, Fibrés vectoriels sur les courbes algébriques, Astérisque 96 (1982). MR 85b:14023

26. C.S. Seshadri, Vector bundles on curves, Contemp. Math. 153 (1993), 163-200. MR 95b:14008

27. C. Simpson, Moduli of representations of the fundamental group of a smooth projective variety I, Inst. Hautes Études Sci. Publ. Math. 79 (1994), 47-129. MR 96e:14012

Instituto de Matemática Pura e Aplicada, Estrada Dona Castorina 110, 22460-320 Rio de JANEIRo RJ, BRAZIL

E-mail address: esteves@impa.br 